\title{
Ordinary state-based peridynamic model for geometrically nonlinear analysis
}

\section{Cong Tien Nguyen and Selda Oterkus*}

Department of Naval Architecture, Ocean and Marine Engineering, University of Strathclyde, Glasgow, G11XQ, UK

\begin{abstract}
This study presents a novel ordinary state-based peridynamic model for geometrically nonlinear analysis. A new definition of logarithmic bond stretch for large deformations has been proposed. The peridynamic formulations for one-dimensional, two-dimensional and three-dimensional structures are obtained based on the principle of virtual displacements by using Total Lagrange formulation. The capability of the developed peridynamic model is demonstrated by predicting large deformations for a bar, a plate, and a three-dimensional structure. To further demonstrate the capabilities of the proposed model, damage on a plate and a three-dimensional structure are simulated.
\end{abstract}

Keywords: peridynamics, nonlinear, large deformations, Total Lagrange, damage 


\section{Nomenclature}

\section{Latin Letters}

A

$A_{0}$

$a, b, d$

$\mathbf{b}_{(k)}=\left[\begin{array}{lll}b_{x(k)} & b_{y(k)} & b_{z(k)}\end{array}\right]^{T}$

${ }_{0}^{t} \mathbf{C}$

$c_{(k)(j)}$

${ }_{0}^{t} C_{i j r s}$

E

${ }_{0}^{t} \mathbf{E}^{H}$

${ }_{0}^{t} E_{m}^{H}$

${ }_{0}^{t} \mathbf{E}_{d}^{H}$

${ }^{t+\Delta t} e_{i j}$

${ }_{0}^{t} \mathbf{F}$

${ }^{t+\Delta t} \mathbf{F}_{e x t}$

${ }^{t} \mathbf{F}_{\text {ext }}$

${ }^{t+\Delta t} \mathbf{f}^{B}$

${ }^{t+\Delta t} \mathbf{f}^{S}$

G

$G_{c}$

$g^{c}$

$\bar{g}_{(k)(j)}$

$\mathbf{H}_{0}$

h

$\mathbf{K}_{(k)}$

${ }_{0}^{t} \mathbf{K}_{L},{ }_{0}^{t} \mathbf{K}_{N L}$

$J^{-}$

$K^{+}$

L

M
The cross-section area of a one-dimensional structure Unit crack surface

Peridynamic constants

Vector of body force

Right Cauchy-Green deformation tensor

A coefficient which depends on the location of the intersection between the line of interaction and the crack surface, $A_{0}$.

Incremental stress-strain tensor at time $t$ with respect to the initial configuration.

Young's modulus

Hencky strain tensor

Mean strain

Deviatoric strain tensor

Incremental linear strain from time $t$ to time $t+\Delta t$, with respect to the initial configuration.

Vector of point forces equivalent to the element stresses at time $t$

Vector of externally applied point loads at time $t+\Delta t$

Vector of externally applied point loads at time $t$ used in explicit time integration.

External body force

External surface force

Total energy release of all interactions passing through the unit crack surface, $A_{0}$

Critical energy release rate of material

Average critical energy release rate for one interaction

Energy release rate for interaction between material points $k$ and $j$ in peridynamics

Angular momentum

Plate thickness

Peridynamic shape function

Linear and nonlinear stiffness matrices, respectively.

Number of material points below the crack surface within the family of $\mathbf{x}_{\left(k^{+}\right)}$whose line of interaction with $\mathbf{x}_{\left(k^{+}\right)}$crosses the crack surface

Number of material points above the crack surface within the family of $\mathbf{x}_{\left(j^{-}\right)}$whose line of interaction with $\mathbf{x}_{\left(k^{+}\right)}$crosses the crack surface

Linear momentum

Time-independent mass matrix 


\begin{tabular}{|c|c|}
\hline$N_{c}$ & $\begin{array}{l}\text { Total number of interactions passing through a unit crack } \\
\text { surface }\end{array}$ \\
\hline $\mathbf{P}$ & Eigenvectors of right Cauchy-Green deformation tensor, ${ }_{0}^{t} \mathbf{C}$ \\
\hline${ }_{0}^{t} \mathbf{R}$ & Rotational matrix \\
\hline${ }^{t+\Delta t} \mathfrak{R}$ & External virtual work \\
\hline${ }^{t+\Delta t} S$ & Surface on which the surface force is applied \\
\hline${ }_{0}^{t} \mathbf{S}$ & Second Piola-Kirchhoff stress tensor \\
\hline${ }_{\tau_{0}}^{t+\Delta t} S_{i j}$ & $\begin{array}{l}\text { Components of Second Piola-Kirchhoff (SPK) stress with } \\
\text { respect to configuration at time } \tau_{0} \text {. }\end{array}$ \\
\hline${ }_{0}^{t} S_{i j},{ }_{0}^{t+\Delta t} S_{i j}$ & $\begin{array}{l}\text { Components of Second Piola-Kirchhoff (SPK) stress at time } t \\
, t+\Delta t \text {, respectively. They are used in Total Lagrange } \\
\text { formulation. }\end{array}$ \\
\hline${ }^{t+\Delta t} S_{i j}$ & Incremental value of SPK stress from time $t$ to time $t+\Delta t$ \\
\hline$s$ & Linear bond stretch \\
\hline$s_{H}$ & Logarithmic bond stretch \\
\hline $\mathbf{t}, \mathbf{t}^{\prime}$ & Peridynamic force densities \\
\hline$\hat{\mathbf{U}}$ & Total vector of increments for displacements \\
\hline${ }^{t+\Delta t} \hat{\ddot{\mathbf{U}}}$ & Total vector of acceleration at time $t+\Delta t$. \\
\hline${ }_{0}^{t} \mathbf{U}$ & Right stretch tensor \\
\hline $\mathbf{u}, \ddot{\mathbf{u}}$ & Displacement and acceleration vectors \\
\hline$u, v, w$ & Displacements in $x, y$ and $z$ directions, respectively \\
\hline${ }_{t}^{t+\Delta t} u_{i}$ & Incremental displacement $u_{i}$ from time $t$ to time $t+\Delta t$ \\
\hline$V_{(j)}$ & Volume of material point $j$ \\
\hline$\left({ }^{0} x,{ }^{0} y,{ }^{0} z\right)$ & Coordinates in the initial (undeformed) configuration \\
\hline$\left({ }^{t} x,{ }^{t} y,{ }^{t} z\right)$ & Coordinates in the current (deformed) configurations \\
\hline$W_{(k)}^{P D}$ & Strain energy density in peridynamics \\
\hline${ }_{0}^{t} \mathbf{X}$ & Deformation gradient \\
\hline${ }_{0}^{t} \mathbf{X}^{\prime}$ & Deformation gradient in the principal coordinates \\
\hline
\end{tabular}

\section{Greek Letters}

$$
\begin{aligned}
& \delta^{t+\Delta t} e_{i j} \\
& \delta_{H} \\
& \delta W^{C C M} \\
& \delta W^{P D} \\
& { }^{t+\Delta t}{ }_{{ }_{0}}{ }{ }_{i j} \\
& { }^{t+\Delta t}{ }^{t} \eta_{i j} \\
& \kappa \\
& \lambda, \mu
\end{aligned}
$$

Total number of interactions passing through a unit crack surface

Peridynamic force densities

Right stretch tensor

Displacement and acceleration vectors

Displacements in $x, y$ and $z$ directions, respectively

Incremental displacement $u_{i}$ from time $t$ to time $t+\Delta t$

Virtual value of strain at time $t+\Delta t$

Horizon size

Virtual value of strain energy density corresponding to virtual displacements in classical continuum mechanics.

Virtual value of strain energy density in peridynamics.

Components of Green Lagrange (GL) strain with respect to configuration at time $\tau_{0}$.

Incremental nonlinear strain from time $t$ to time $t+\Delta t$, with respect to initial configuration.

Bulk modulus of material

Lame's constants 


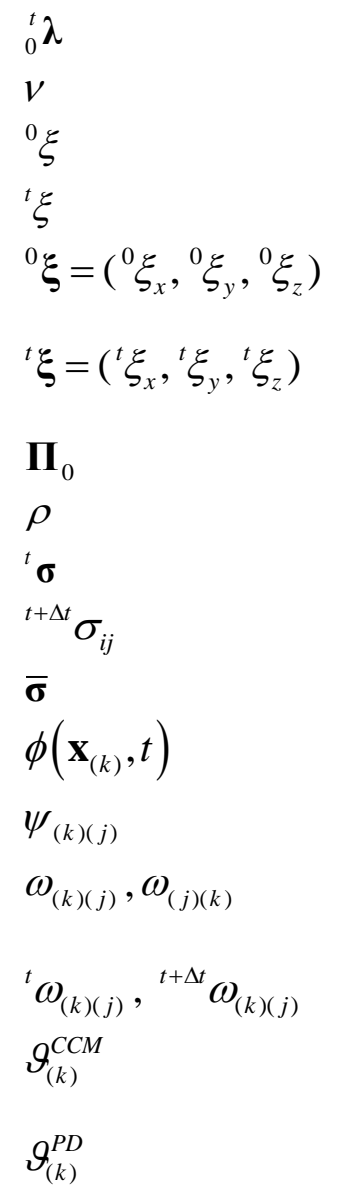

\section{Acronyms}

CCM

FEA

GL

NLFEA

NLPD

PD

SED

SPK

TL/UL

1D, 2D, 3D
Eigenvalues of right Cauchy-Green deformation tensor, ${ }_{0}^{t} \mathbf{C}$

Poisson's ratio

Bond length in initial (undeformed) configuration

Bond length in deformed configuration

Relative coordinates between two material points in the initial configuration

Relative coordinates between two material points in the deformed configurations

Total torque acting on the PD model

Mass density

Cauchy stress

The Cartesian components of Cauchy stress tensor

Stress measure

Local damage coefficient

Function to represent state of interaction (damaged or intact)

Micropotentials of the interaction between material point $k$ and $j$

Micropotential at current time $t, t+\Delta t$, repsectively

Volumetric strain of material point $k$ in classical continuum mechanics

Volumetric strain of material point $k$ in peridynamics

Classical Continuum Mechanics

Finite Element Analysis

Green Lagrange strain

Nonlinear Finite Element Analysis

Nonlinear Peridynamics

Peridynamics

Strain Energy Density

Second Piola-Kirchhoff stress

Total Lagrange/ Updated Lagrange

One, two, three dimensions 


\section{Introduction}

Structures can exhibit large deformations when subjected to large loads. In these cases, the linear analysis which is based on a small deformation assumption may not give good results. Therefore, an appropriate nonlinear solution is needed. When the deformation is large, the structure's behaviors can be elastic or inelastic. A fundamental difference between elastic and inelastic analysis is that in elastic solution the total stress can be directly evaluated from total strain, whereas in the inelastic analysis the stress and strain history is also included in the calculation of total stress.

In nonlinear finite element analysis (FEA), the equation of motion is obtained from the linearization of the principle of virtual displacements [1]. The solution in the nonlinear analysis is obtained after several iterations as opposed to linear FEA. These types of problems are well understood within the Classical Continuum Mechanics (CCM), as well as finite element analyses [1-9]. However, because of using partial-differential equations that become invalid in presence of discontinuities, the classical continuum mechanics face mathematical and conceptual difficulties in terms of predicting crack nucleation and growth, especially for multiple crack paths.

Peridynamics (PD) represents the material behavior by using integro-differential equations, which are valid in both continuous and discontinuous models [10]. Therefore, discontinuities can be naturally involved in the PD analysis without any special treatment. The first bond based PD model was introduced by Silling [10] and Silling and Askari [11]. Later, Silling et al. [12], Silling and Lehoucq [13] introduced a state-based PD model which can overcome the limitation of bond based PD model in term of Poisson's ratio. Recently, a 3-D conjugated bond-pairbased peridynamics model is proposed to overcome the limitation of the fixed Poisson's ratio $[14,15]$. Bergel and Li [16] introduced total and updated Lagrangian formulations of statebased peridynamics. Nikravesh and Gerstle introduced an improved state-based peridynamic lattice model including elasticity, plasticity, and damage [17]. An extensive literature survey on peridynamics is given by Madenci and Oterkus [18] and Javili et al. [19].

Peridynamics is applicable in both elastic and plastic material responses [20-23]. Moreover, it can be applied for multiphysics [24-33] and multiscale modeling [34, 35]. In addition, it can also be used to analyze composite and polycrystalline materials [18, 36-41]. PD can also be used to analyze complex structures such as ship and offshore structures by using simplified PD models for beam structures [42, 43] and shell structures [44, 45]. Moreover, it can also be either combined with finite element analysis (FEA) [46-49] or implemented in the FEA framework [50-53].

As discussed by Madenci and Oterkus [18], Cauchy stress components can be expressed in terms of PD force densities. In classical continuum mechanics, Cauchy stress is calculated based on the deformed configuration which is affected by volume changes and rotations for large deformation problems. Therefore, rotations and volume changes can also affect the calculation of PD force densities. In current PD literature, the force densities, in bond-based and ordinary state-based PD models, are parallel to the line of interaction on deformed configuration. However, the effect of volume change is excluded from the calculation of PD force densities. Therefore, for large deformation problems in which the effects of volume change are significant, a new formulation of PD force densities, which considers both effects of rotation and volume change, need to be used. This study focuses on developing a nonlinear PD model in which the force densities are parallel to the line of interaction on deformed configuration and depend on the change of bond length. 
The logarithmic bond stretch is defined and used for the first time in the PD literature. The PD formulation and equations of motion are obtained based on the principle of virtual displacements by using Total Lagrange formulation. The numerical procedure for PD nonlinear analysis is provided. The capabilities of the PD model are verified by considering 1D, 2D, and 3D structures subjected to large deformations. The developed nonlinear PD model is used to predict damages in 2D plates and 3D structures. The damage predictions are compared with experimental results conducted by Kalthoff [54, 55], Kalthoff and Winkler [56], Jenq and Shah [57].

This paper is organized as follows. Section 2 presents the general form of PD equations of motion and constitutive relations. Section 3 presents the PD equation of motion for large deformation problems. The damage criterion for PD damage prediction based on critical energy release rate is provided in Section 4 and numerical procedure is presented in Section 5. Section 6 presents numerical results. Finally, the conclusions are presented in Section 7.

\section{Peridynamic equation of motion}

The peridynamic equation of motion introduced by Silling [10] and later generalized by Silling et al. [12] is an integro-differential equation in time and space. The PD equation of motion is described as

$$
\rho(\mathbf{x}) \ddot{\mathbf{u}}(\mathbf{x}, t)=\int_{H_{x}}\left(\mathbf{t}\left(\mathbf{u}^{\prime}-\mathbf{u}, \mathbf{x}^{\prime}-\mathbf{x}, t\right)-\mathbf{t}^{\prime}\left(\mathbf{u}-\mathbf{u}^{\prime}, \mathbf{x}-\mathbf{x}^{\prime}, t\right)\right) d V^{\prime}+\mathbf{b}(\mathbf{x}, t)
$$

where $\rho$ represents mass density, $\mathbf{b}(\mathbf{x}, t)$ represents the body force, $\mathbf{u}$ and $\ddot{\mathbf{u}}$ represent displacement and acceleration, respectively. The parameter $\mathbf{t}$ represents the force density that material point at $\mathbf{x}^{\prime}$ exerts on $\mathbf{x}$ and $\mathbf{t}^{\prime}$ represents the force density that material point at $\mathbf{x}$ exerts on material point at $\mathbf{x}^{\prime}$. In PD theory, a material point located at coordinates $\mathbf{x}$ has interactions with its surrounding points within a distance $\delta_{H}$ which called horizon size. The sphere (in a three-dimensional body) or the circle (in a two-dimensional body) surrounding material point $\mathbf{x}$ with the radius of $\delta_{H}$ is called the horizon of material point, denoted as $H_{x}$.

In discrete form, Eq. (1) can be represented as

$$
\rho_{(k)} \ddot{\mathbf{u}}_{(k)}=\sum_{j=1}^{N}\left(\mathbf{t}\left(\mathbf{u}_{(j)}-\mathbf{u}_{(k)}, \mathbf{x}_{(j)}-\mathbf{x}_{(k)}, t\right)-\mathbf{t}^{\prime}\left(\mathbf{u}_{(k)}-\mathbf{u}_{(j)}, \mathbf{x}_{(k)}-\mathbf{x}_{(k)}, t\right)\right) V_{(j)}+\mathbf{b}_{(k)}
$$

with

$$
\begin{aligned}
& \mathbf{t}\left(\mathbf{u}_{(j)}-\mathbf{u}_{(k)}, \mathbf{x}_{(j)}-\mathbf{x}_{(k)}, t\right)=\mathbf{t}_{(k)(j)}=\left[\begin{array}{lll}
t_{x(k)(j)} & t_{y(k)(j)} & t_{z(k)(j)}
\end{array}\right]^{T} \\
& \mathbf{t}^{\prime}\left(\mathbf{u}_{(k)}-\mathbf{u}_{(j)}, \mathbf{x}_{(k)}-\mathbf{x}_{(k)}, t\right)=\mathbf{t}_{(j)(k)}=\left[\begin{array}{lll}
t_{x(j)(k)} & t_{y(j)(k)} & t_{z(j)(k)}
\end{array}\right]^{T} \\
& \mathbf{b}_{(k)}=\left[\begin{array}{lll}
b_{x(k)} & b_{y(k)} & b_{z(k)}
\end{array}\right]^{T}
\end{aligned}
$$

where $N$ represents the total number of family members of material point $k$. Here, $k$ represents material point located at coordinates $\mathbf{x}$.

The force densities given in Eq. (2) can be calculated by using the relation between PD strain energy density, $W_{(k)}^{P D}$ and PD force density developed by Madenci and Oterkus [18] as

$$
\mathbf{t}_{(k)(j)}=-\frac{1}{V_{(j)}} \frac{\partial W_{(k)}^{P D}}{\partial \mathbf{u}_{(k)}}, \mathbf{t}_{(j)(k)}=-\frac{1}{V_{(k)}} \frac{\partial W_{(j)}^{P D}}{\partial \mathbf{u}_{(j)}}
$$




\section{Peridynamics equation of motion for large deformations}

By using similar PD strain energy density expressions defined in $[11,12,18]$, the PD strain energy density for large deformations can be described as

$W_{(k)}^{P D}=a\left(\vartheta_{(k)}^{P D}\right)^{2}+b \sum_{j=1}^{N} s_{H}^{2}{ }^{0} \xi V_{(j)}$ for 3D and 2D

$W_{(k)}^{P D}=b \sum_{j=1}^{N} s_{H}^{2}{ }^{0} \xi V_{(j)}$ for $1 \mathrm{D}$

Here $\vartheta_{(k)}^{P D}$ represents the PD volumetric strain, $s_{H}$ represents the logarithmic bond stretch and $V_{(j)}$ represents the volume of material point $j$ in the undeformed configuration. The parameters $a$ and $b$ represent the peridynamic constants.

The logarithmic bond stretch, $s_{H}$, is defined similarly to Hencky strain definition [2] for large deformation problems as [1]

$s_{H}=\ln (1+s)=\ln \left(\frac{{ }^{t} \xi}{{ }^{0} \xi}\right)$

with

$$
\begin{aligned}
& { }^{0} \xi=\sqrt{{ }^{0} \xi_{x}{ }^{2}+{ }^{0} \xi_{y}{ }^{2}+{ }^{0} \xi_{z}^{2}}=\sqrt{\left({ }^{0} x_{(j)}-{ }^{0} x_{(k)}\right)^{2}+\left({ }^{0} y_{(j)}-{ }^{0} y_{(k)}\right)^{2}+\left({ }^{0} z_{(j)}-{ }^{0} z_{(k)}\right)^{2}} \\
& { }^{t} \xi=\sqrt{{ }^{t} \xi_{x}{ }^{2}+{ }^{t} \xi_{y}{ }^{2}+{ }^{t} \xi_{z}{ }^{2}}=\sqrt{\left({ }^{t} x_{(j)}-{ }^{t} x_{(k)}\right)^{2}+\left({ }^{t} y_{(j)}-{ }^{t} y_{(k)}\right)^{2}+\left({ }^{t} z_{(j)}-{ }^{t} z_{(k)}\right)^{2}}
\end{aligned}
$$

where ${ }^{t} \xi$ and ${ }^{0} \xi$ represent the current length and initial length of the bond between two material points as shown in Fig. 1 . Here $\left({ }^{0} x,{ }^{0} y,{ }^{0} z\right)$ and $\left({ }^{t} x,{ }^{t} y,{ }^{t} z\right)$ represent coordinates in the initial (undeformed) and current configurations, respectively. Whereas, $\left({ }^{0} \xi_{x},{ }^{0} \xi_{y},{ }^{0} \xi_{z}\right)$ and $\left({ }^{t} \xi_{x},{ }^{t} \xi_{y},{ }^{t} \xi_{z}\right)$ represent relative coordinates between two material points in the initial and current configurations, respectively. 


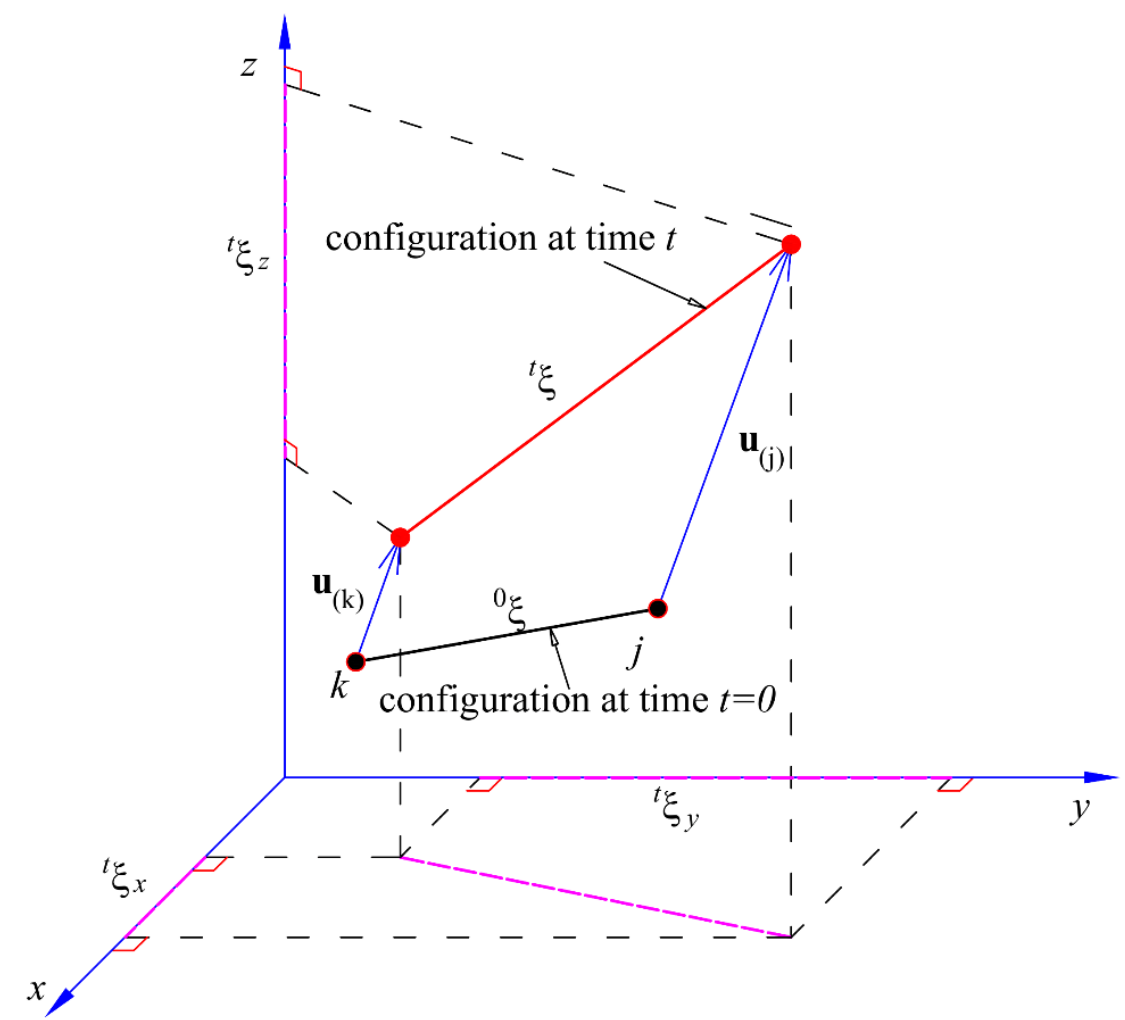

Fig. 1. Initial and deformed configuration of an interaction

In Eq. (5a), the linear form of stretch, $s$ is defined according to Silling and Askari [11] as $s=\frac{{ }^{t} \xi-{ }^{0} \xi}{{ }^{0} \xi}$

Similar to classical form, for small deformations, $s \ll 1$, the logarithmic bond stretch reduces to its linear form as

$s_{H}=\ln (1+s) \approx s$

The PD volumetric strain term in Eq. (4a) can be calculated as

$\vartheta_{(k)}^{P D}=d \sum_{j=1}^{N} s_{H} V_{(j)}$

where $s_{H}$ represents logarithmic bond stretch as defined in Eq. (5), $d$ represents peridynamic constant.

By substituting strain energy density definitions provided in Eq. (4a) into Eq. (3), the force density components that material point $j$ exerts on material point $k$ can be represented as

$\mathbf{t}_{(k)(j)}=\left(2 a d \frac{1}{{ }^{0} \xi} \vartheta_{(k)}^{P D}+2 b s_{H}\right) \frac{{ }^{0} \xi}{{ }^{t} \xi} \frac{{ }^{t} \boldsymbol{\xi}}{{ }^{t} \xi}$

Similarly, the force density components that material point $k$ exerts on material point $j$ can be represented as

$\mathbf{t}_{(j)(k)}=-\left(2 a d \frac{1}{{ }^{0} \xi} \vartheta_{(j)}^{P D}+2 b s_{H}\right)^{0} \frac{\xi}{{ }^{t} \xi} \frac{{ }^{t} \xi}{{ }^{t} \xi}$

Note that the force densities given in Eqs. (9-10) are parallel to the deformed configuration and the term ${ }^{0} \xi /{ }^{t} \xi$ represents the ratio between initial and current bond lengths. 
By substituting Eqs. (9-10) into Eq. (2), the nonlinear PD equation of motion can be obtained as

For 3D and 2D:

$\rho \ddot{\mathbf{u}}_{(k)}=\sum_{j=1}^{N}\left(2 a d \frac{1}{{ }^{0} \xi}\left(\vartheta_{(k)}^{P D}+\vartheta_{(j)}^{P D}\right)+4 b s_{H}\right) \frac{{ }^{0} \xi}{{ }^{t} \xi} \frac{{ }^{t} \xi}{{ }^{t} \xi} V_{(j)}+\mathbf{b}_{(k)}$

For 1D:

$\rho \ddot{u}_{(k)}=\sum_{j=1}^{N} 4 b s_{H} \frac{{ }^{0} \xi}{{ }^{t} \xi} \frac{{ }^{t} \xi_{x}}{{ }^{t} \xi} V_{(j)}+b_{x(k)}$

The PD constants, $a$ and $b$ are determined by comparing the virtual values of strain energy density in PD and classical continuum mechanics based on the principle of virtual displacement provided in Appendix A.

The peridynamic constants, $a, b$ and $d$ for 3D structures are obtained as (Appendix B)

$a=\frac{\lambda-\mu}{2}$

$b=\frac{15}{2} \frac{\mu}{\pi \delta_{H}^{4}}$

$d=\frac{9}{4 \pi \delta_{H}^{3}}$

where $\delta_{H}$ represents horizon size in the initial configuration, $\lambda$ and $\mu$ represent Lame's constants

$\lambda=\frac{E v}{(1+v)(1-2 v)} ; \mu=\frac{E}{2(1+v)}$

where $E$ represents the elastic modulus and $v$ represents the Poisson's ratio.

The peridynamic constants, $a, b$ and $d$ for 2D structures are obtained as (Appendix C)

$a=\frac{\lambda \alpha-\mu}{2}$

$b=\frac{6 \mu}{\pi h \delta_{H}^{3}}$

$d=\frac{2}{\pi h \delta_{H}^{2}}$

where $h$ represents the thickness of the plate in the initial configuration, $\alpha$ represents a constant which can be defined as

$\alpha= \begin{cases}\frac{1-2 v}{1-v} & \text { for plane stress } \\ 1 & \text { for plane strain }\end{cases}$

As provided in Appendix D, peridynamic constants for 1D structures can be obtained as

$b=\frac{E}{2 A \delta_{H}^{2}}$

where $A$ represents the cross-section area of the bar in the initial configuration. 
Note that the PD constants provided in Eq. (13), Eq. (15) and Eq. (17) agree to those given in [18]. Therefore, it can be concluded that the key difference between PD formulations for small deformation problems and for large deformation problems is the use of logarithmic bond stretch provided in Eq. (7). As a result, the change of bond length is included in the formulations of PD force densities as given in Eqs. (9-10).

\section{Damage prediction}

Damage prediction in peridynamics is introduced by eliminating interactions between material points. The force densities between material points are irreversibly removed when the interaction is considered as broken and it leads to crack growth. The state of interaction, intact or broken, is represented by parameter $\psi$ which can be described as [11]

$\psi_{(k)(j)}\left(\mathbf{x}_{(j)}-\mathbf{x}_{(k)}, t\right)=\left\{\begin{array}{l}1 \text { if interaction exists } \\ 0 \text { if no interaction }\end{array}\right.$

Therefore, the peridynamic equations of motion given in Eqs. (11-12) can be rewritten as

$\rho_{(k)} \ddot{\mathbf{u}}_{(k)}=\sum_{j=1}^{N} \psi_{(k)(j)} \mathbf{f}_{(k)(j)} V_{(j)}+\mathbf{b}_{(k)}$

where

$$
\begin{aligned}
& \mathbf{f}_{(k)(j)}= {\left[\begin{array}{l}
\left(2 a d \frac{1}{{ }^{0} \xi}\left(\vartheta_{(k)}^{P D}+\vartheta_{(j)}^{P D}\right)+4 b s_{H}\right) \frac{{ }^{0} \xi}{{ }^{t} \xi} \frac{{ }^{t} \xi_{x}}{{ }^{t} \xi} \\
\left(2 a d \frac{1}{{ }^{0} \xi}\left(\vartheta_{(k)}^{P D}+\vartheta_{(j)}^{P D}\right)+4 b s_{H}\right) \frac{{ }^{0} \xi}{{ }^{t} \xi} \frac{{ }^{t} \xi}{{ }^{t} \xi} \\
\left(2 a d \frac{1}{{ }^{0} \xi}\left(\vartheta_{(k)}^{P D}+\vartheta_{(j)}^{P D}\right)+4 b s_{H}\right) \frac{{ }^{0} \xi}{{ }^{t} \xi} \frac{{ }^{t} \xi}{{ }^{t} \xi}
\end{array}\right] \text { for 3D } } \\
& \mathbf{f}_{(k)(j)}=\left[\begin{array}{l}
\left(2 a d \frac{1}{{ }^{0} \xi}\left(\vartheta_{(k)}^{P D}+\vartheta_{(j)}^{P D}\right)+4 b s_{H}\right) \frac{{ }^{0} \xi}{{ }^{t} \xi} \frac{{ }^{t} \xi_{x}}{{ }^{t} \xi} \\
\left(2 a d \frac{1}{{ }^{0} \xi}\left(\vartheta_{(k)}^{P D}+\vartheta_{(j)}^{P D}\right)+4 b s_{H}\right) \frac{{ }^{0} \xi}{{ }^{t} \xi} \frac{{ }^{t} \xi}{{ }^{t} \xi}
\end{array}\right] \text { for } 2 \mathrm{D} \\
& \mathbf{f}_{(k)(j)}=4 b s_{H} \frac{{ }^{0} \xi}{{ }^{t} \xi} \frac{{ }^{t} \xi}{{ }^{t} \xi} \text { for } 1 \mathrm{D}
\end{aligned}
$$

Note that, the volumetric strain in PD for damage prediction can be updated by including the damage parameter, $\psi$ as

$$
\vartheta_{(k)}^{P D}=d \sum_{j=1}^{N} \psi_{(k)(j)} s_{H} V_{(j)}
$$

The local damage coefficient, $\phi$, which can be defined as [11]

$$
\phi\left(\mathbf{x}_{(k)}, t\right)=1-\frac{\sum_{j=1}^{N} \psi_{(k)(j)}\left(\mathbf{x}_{(j)}-\mathbf{x}_{(k)}, t\right) V_{(j)}}{\sum_{j=1}^{N} V_{(j)}}
$$

In order to determine the state of interaction, there are criteria based on either the critical stretch $[11,18]$ or critical energy rate [22, 23, 58] or stress-based strength [59]. The criteria based on the critical energy rate can be described as 
$\bar{g}_{(k)(j)}<g^{c} \rightarrow$ interaction exists: $\psi_{(k)(j)}=1$

$\bar{g}_{(k)(j)} \geq g^{c} \rightarrow$ interaction is broken: $\psi_{(k)(j)}=0$

where $\bar{g}_{(k)(j)}$ represents the energy release rate for interaction between material points $k$ and $j$,

$g^{c}$ represents the critical energy release rate for one interaction. The critical energy release rate for one interaction, $g^{c}$, can be determined by using the criterion based on discrete critical energy density $[22,23]$ or by using the criterion based on continuum critical energy density [58]. In this study, the criterion based on discrete critical energy density [22, 23] is used.

As shown in Fig. 2, the material points $\mathbf{x}_{\left(k^{+}\right)}$and $\mathbf{x}_{\left(j^{-}\right)}$are located above and below the unit crack surface $A_{0}$, respectively. The total energy release of all interactions across the unit crack surface, $A_{0}$, can be represented as [22, 23]

$G=\frac{1}{2} \sum_{k=1}^{K^{+}} \sum_{j=1}^{J^{-}} \frac{1}{2}\left(g_{\left(k^{+}\right)\left(j^{-}\right)}+g_{\left(j^{-}\right)\left(k^{+}\right)}\right) c_{(k)(j)}$

where $g_{\left(k^{+}\right)\left(j^{-}\right)}$and $g_{\left(j^{-}\right)\left(k^{+}\right)}$represent energy release rate for interaction between material points $k^{+}$and $j^{-}$. The parameter $c_{(k)(j)}$ represents a coefficient that depends on the location of the line of interaction between material points $k^{+}$and $j^{-}$, and the crack surface. In Eq. (23), parameter $J^{-}$represents the number of family members of material point $\mathbf{x}_{\left(k^{+}\right)}$which are below the crack surface and whose line of interactions passing through the crack surface. The parameter $K^{+}$represents the number of family members of material point $\mathbf{x}_{\left(j^{-}\right)}$which are above the crack surface and whose line of interactions passing through the crack surface.

For 1D structure, the line of interaction between two material points passes through the unit crack surface which is the cross-sectional area of the bar as shown in Fig. 3. Therefore, the coefficient $c_{(k)(j)}$ is equal to 1 [43]. For 2D structure, the line of interaction between two material points can pass either through the crack tips or through the crack surface as shown in Fig. 4. The interactions passing through the crack tips can be counted as half interaction with $c_{(k)(j)}=1 / 2$, meanwhile the interaction passing through the crack surface can be counted as full interaction with $c_{(k)(j)}=1[22,23,45]$. For 3D structure, line of interaction between two material points can pass through either crack edge, or crack corner, or crack surface, $A_{0}$ as shown in Fig. 5. The interaction passing through the crack surface can be counted as full interaction with $c_{(k)(j)}=1$. The interaction passing through the crack edges can be counted as half interaction with $c_{(k)(j)}=1 / 2$, meanwhile the interaction passing through the crack corners can be counted as quarter interaction with $c_{(k)(j)}=1 / 4$.

In order to eliminate all interactions across the unit crack surface, $A_{0}$, all of the energies between the material points whose line of action passing through the crack surface must be eliminated. Therefore, the required critical energy release rate can be represented as $[22,23]$

$G_{c}=\frac{1}{2} \sum_{k=1}^{K^{+}} \sum_{j=1}^{J^{-}} \frac{1}{2}\left(g_{\left(k^{+}\right)\left(j^{-}\right)}^{c}+g_{\left(j^{-}\right)\left(k^{+}\right)}^{c}\right) c_{(k)(j)}$ 
where $G_{c}$ represents the critical energy release rate of the material.

By assuming that the critical energy release rate, $G_{c}$, of a material point is distributed equally to each interaction passing through the crack surface, the following relation can be obtained as $g_{\left(k^{+}\right)\left(j^{-}\right)}^{c} \approx g_{\left(j^{-}\right)\left(k^{+}\right)}^{c}=g^{c}$

By utilizing the relation given in Eq. (25), the critical energy release rate given in Eq. (24) can be represented as

$G_{c}=\frac{1}{2} \sum_{k=1}^{K^{+}} \sum_{j=1}^{J^{-}} c_{(k)(j)} g^{c}=g^{c}\left(\frac{1}{2} \sum_{k=1}^{K^{+}} \sum_{j=1}^{J^{-}} c_{(k)(j)}\right)$

or

$G_{c}=N_{c} g^{c}$

with

$N_{c}=\frac{1}{2} \sum_{k=1}^{K^{+}} \sum_{j=1}^{J^{-}} c_{(k)(j)}$

where $N_{c}$ represents the total number of interactions passing through a unit crack surface.

Therefore, by using Eq. (26b), the critical energy release rate for one interaction can be estimated as

$$
g^{c}=\frac{G_{c}}{N_{c}}
$$

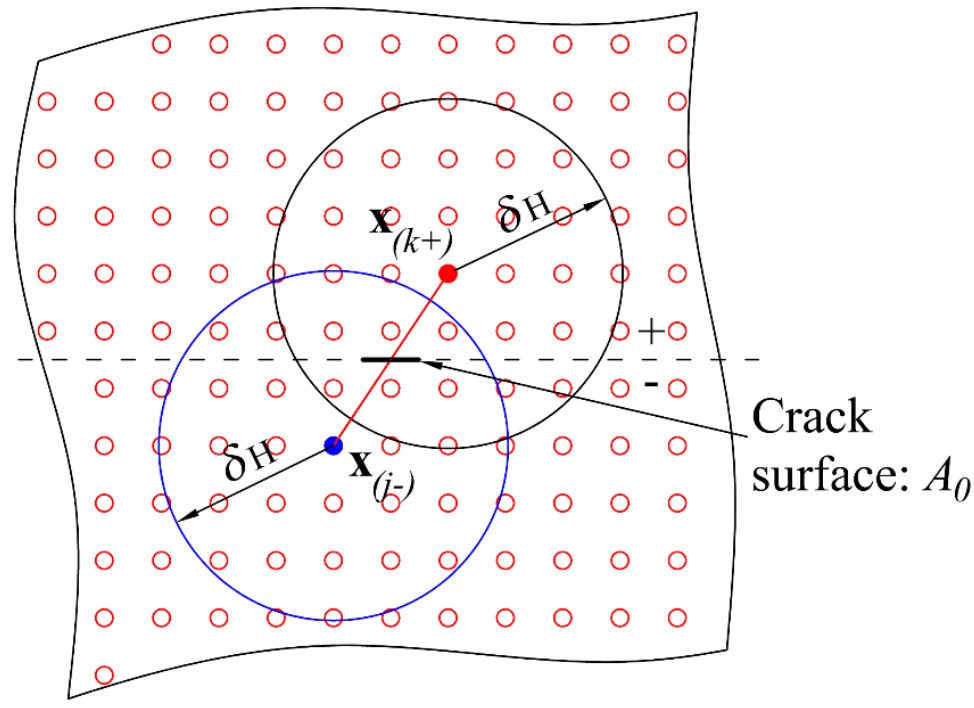

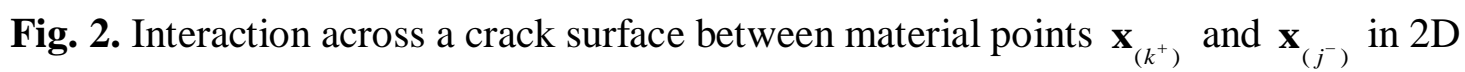
structures

As shown in Fig. 3, there are total $N_{c}=12$ interactions passing through the unit crack surface for 1D structure [43]. As shown in Fig. 4, for 2D structures there are 24 interactions passing through the crack surface and 24 interactions passing through the crack tips. Therefore, total number of interactions passing the unit crack surface can be counted as $N_{c}=24 \times 1+24 \times 1 / 2=36[45]$.

Similarly, for 3D structures, there are 392 interactions passing through the unit crack surface, 320 interactions passing through the crack edges and 32 interactions passing through the crack 
corners. Therefore, total number of interactions passing the unit crack surface can be counted as $N_{c}=392 \times 1+320 \times 1 / 2+32 \times 1 / 4=560$.

Crack surface: $A$
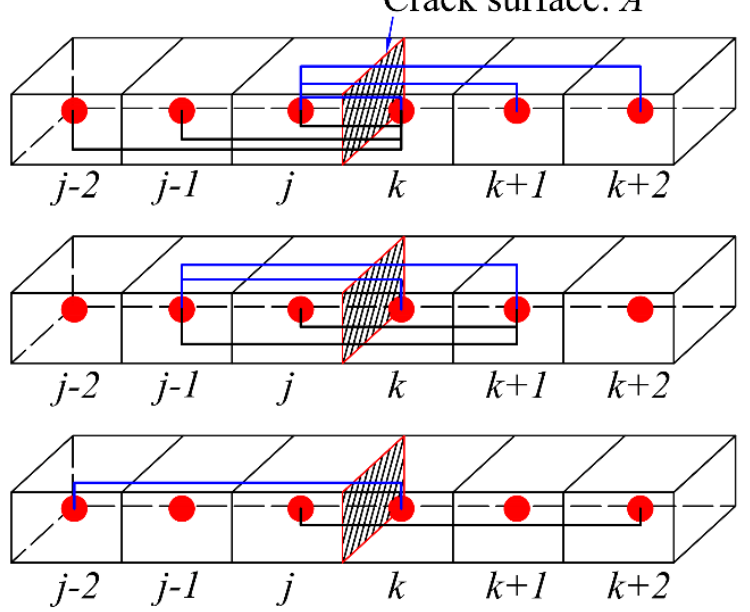

Fig. 3. Counting the number of interaction, $N_{c}$ for $1 \mathrm{D}$ structures for $\delta_{H}=3.015 \Delta x$
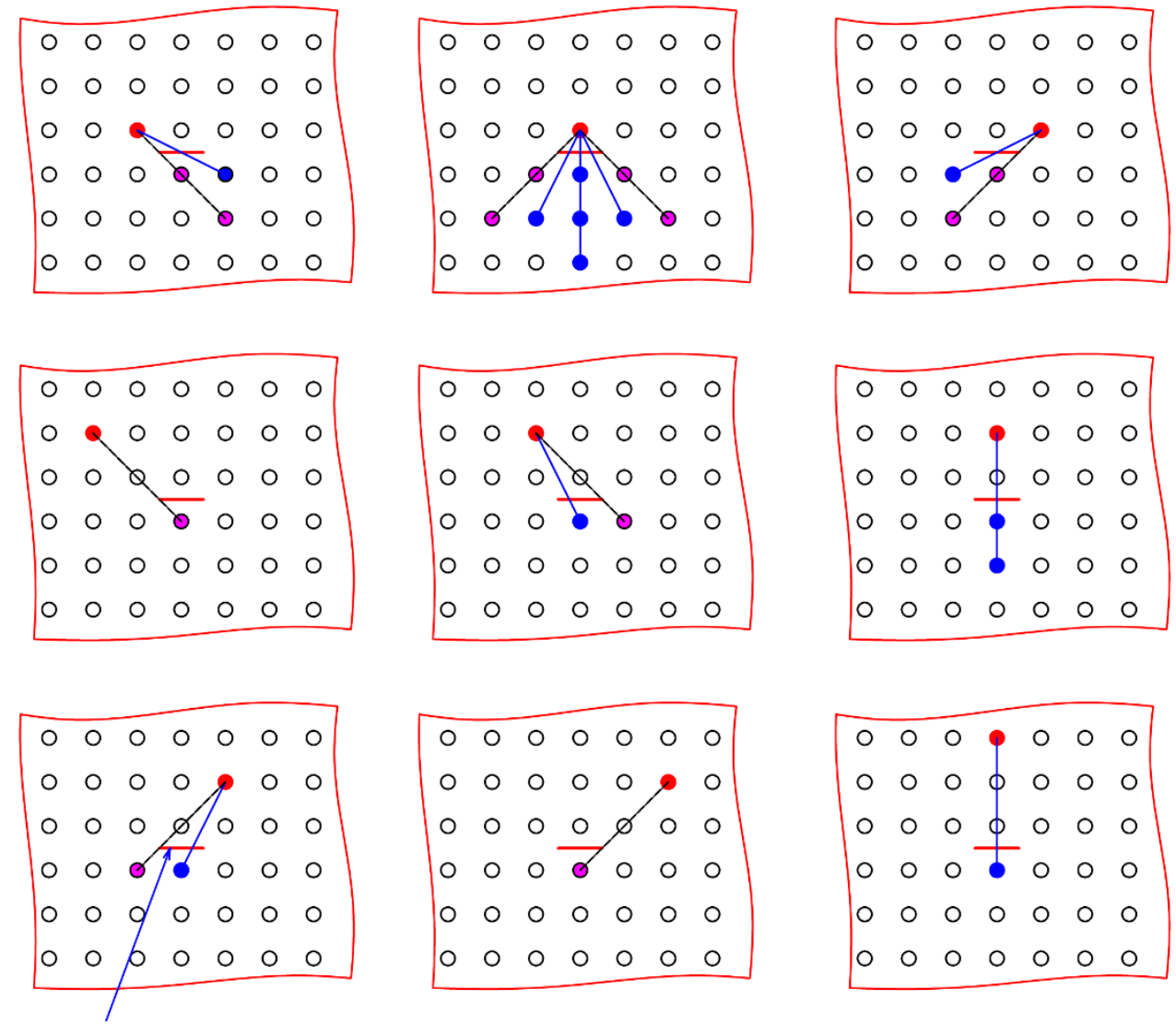

Crack surface, $A$

Interaction is counted as 1 interaction Interaction is counted as $1 / 2$ interaction

Fig. 4. Counting the number of interaction, $N_{c}$, passing unit crack surface on $2 \mathrm{D}$ plate for

$$
\delta_{H}=3.015 \Delta x
$$




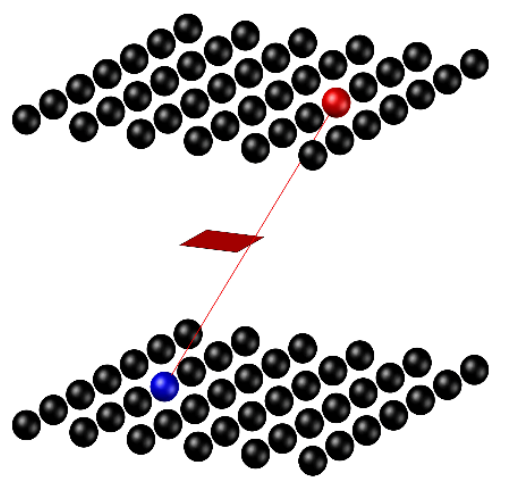

(a)

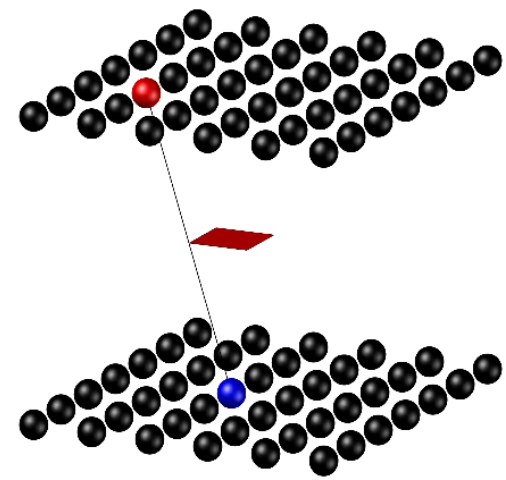

(b)

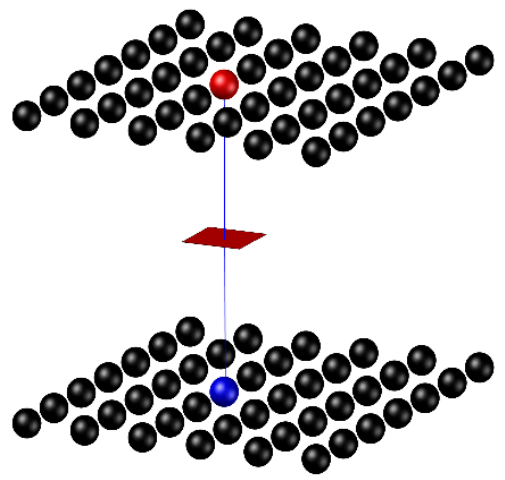

(c)

Fig. 5. Interaction passing (a) crack edges, (b) crack corners, (c) a point inside crack surface on 3D structures for $\delta_{H}=3.015 \Delta x$

In Eq. (22), the energy release rate for the interaction between material points $k$ and $j, \bar{g}_{(k)(j)}$ , can be approximated as $[22,23]$

$$
\bar{g}_{(k)(j)}=\frac{1}{A_{0}} \bar{\omega}_{(k)(j)} V_{(k)} V_{(j)}=\frac{1}{A_{0}}\left(\frac{1}{2}\left(\omega_{(k)(j)}+\omega_{(j)(k)}\right)\right) V_{(k)} V_{(j)}
$$

where $\omega_{(k)(j)}$ represents the micropotential of the interaction between material points $k$ and $j$ in which material point $j$ is a family member of material point $k, \omega_{(j)(k)}$ represents the micropotential of the interaction between material points $j$ and $k$ in which material point $k$ is a family member of material point $j$. The term $\bar{\omega}_{(k)(j)}$ represents the average micropotential of the interaction between material points $k$ and $j$. The term $A_{0}$ represents the unit crack surface as

$$
A_{0}= \begin{cases}A & \text { for 1D structures } \\ \Delta x h & \text { for 2D structures } \\ \Delta x^{2} & \text { for 3D structures }\end{cases}
$$

where $\Delta x$ represents the mesh size for the PD model.

As introduced by Madenci and Oterkus $[22,23]$ the micropotential $\omega_{(k)(j)}$ can be calculated in the nonlinear analysis as

$$
\omega_{(k)(j)}=\int_{0}^{s_{(k)(j)}} t_{(k)(j)} 0 \xi d s=\int_{0_{\xi}}^{t_{\xi}} t_{(k)(j)} d \xi
$$

with

$$
t_{(k)(j)}= \begin{cases}2 b s_{H} \frac{{ }^{0} \xi}{{ }^{t} \xi} & \text { for 1D structures } \\ \left(a d \frac{1}{{ }^{0} \xi}\left(\vartheta_{(k)}^{P D}+\vartheta_{(j)}^{P D}\right)+2 b s_{H}\right) \frac{{ }^{0} \xi}{{ }^{t} \xi} & \text { for 2D and 3D structures }\end{cases}
$$

As shown in Fig. 6, the force density and bond stretch relation is highly nonlinear in the proposed nonlinear PD model. Therefore, the integration in Eq. (30a) can be numerically calculated as

$$
{ }^{t+\Delta t} \omega_{(k)(j)}={ }^{t} \omega_{(k)(j)}+\Delta \omega_{(k)(j)}
$$


where ${ }^{t} \omega_{(k)(j)}$ represents micropotential at the current time step, $t$ and ${ }^{t+\Delta t} \omega_{(k)(j)}$ represents micropotential at the next time step, $t+\Delta t$. The incremental value of micropotential from time $t$ to time $t+\Delta t, \Delta \omega_{(k)(j)}$ can be calculated as

$\Delta \omega_{(k)(j)}=\frac{1}{2}\left({ }^{t+\Delta t} t_{(k)(j)}-{ }^{t} t_{(k)(j)}\right)\left({ }^{t+\Delta t} \xi-{ }^{t} \xi\right)$

where the terms ${ }^{t} t_{(k)(j)}$ and ${ }^{t+\Delta t} t_{(k)(j)}$ represent the force densities, as given in Eq. (30b), at time $t$ and $t+\Delta t$, respectively.

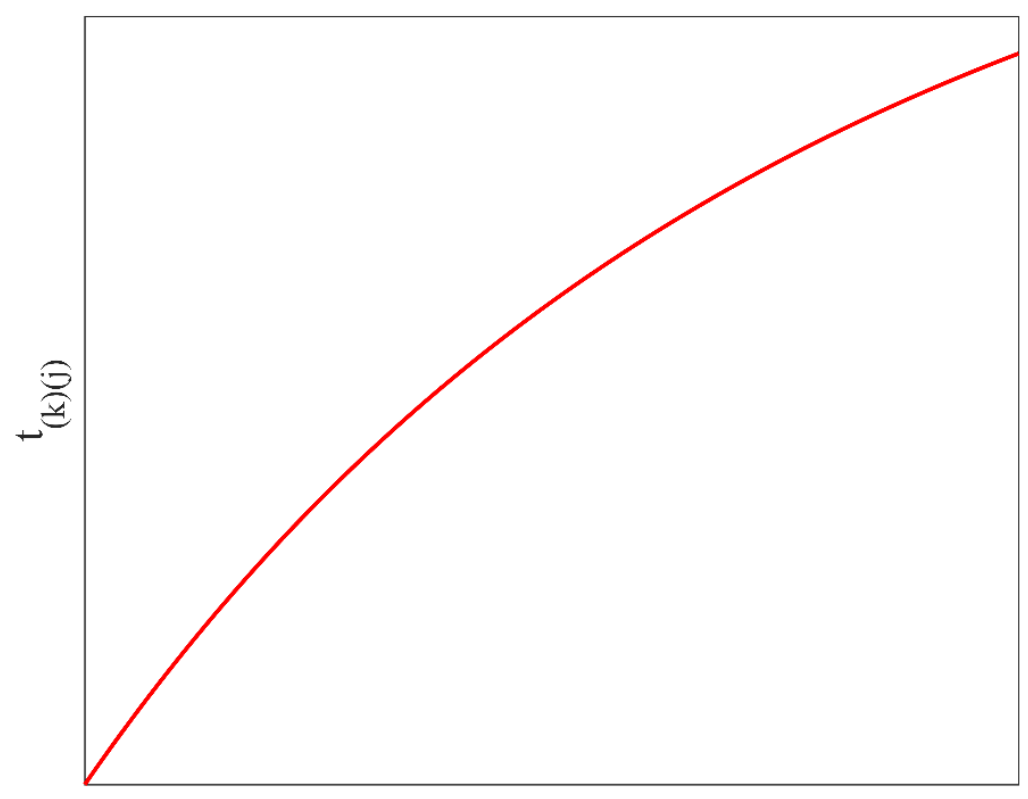

$\mathrm{S}$

Fig. 6. Variations of force density, $t_{(k)(j)}$, as a function of linear bond stretch $s$ for large deformations

\section{Numerical Procedure}

The equations of motion in PD can be solved by using a meshless scheme. The domain is divided into a uniform mesh, with material points associated with specific volumes. The numerical procedure in PD predictions for large deformation is shown in Fig. 7.

Similar to finite element analyses as explained in Appendix A, the material volume can be changed due to large deformation. Therefore, if the boundary condition such as body forces or surface forces are applied, these loading conditions need to be updated during the simulations as described in Appendix E. 


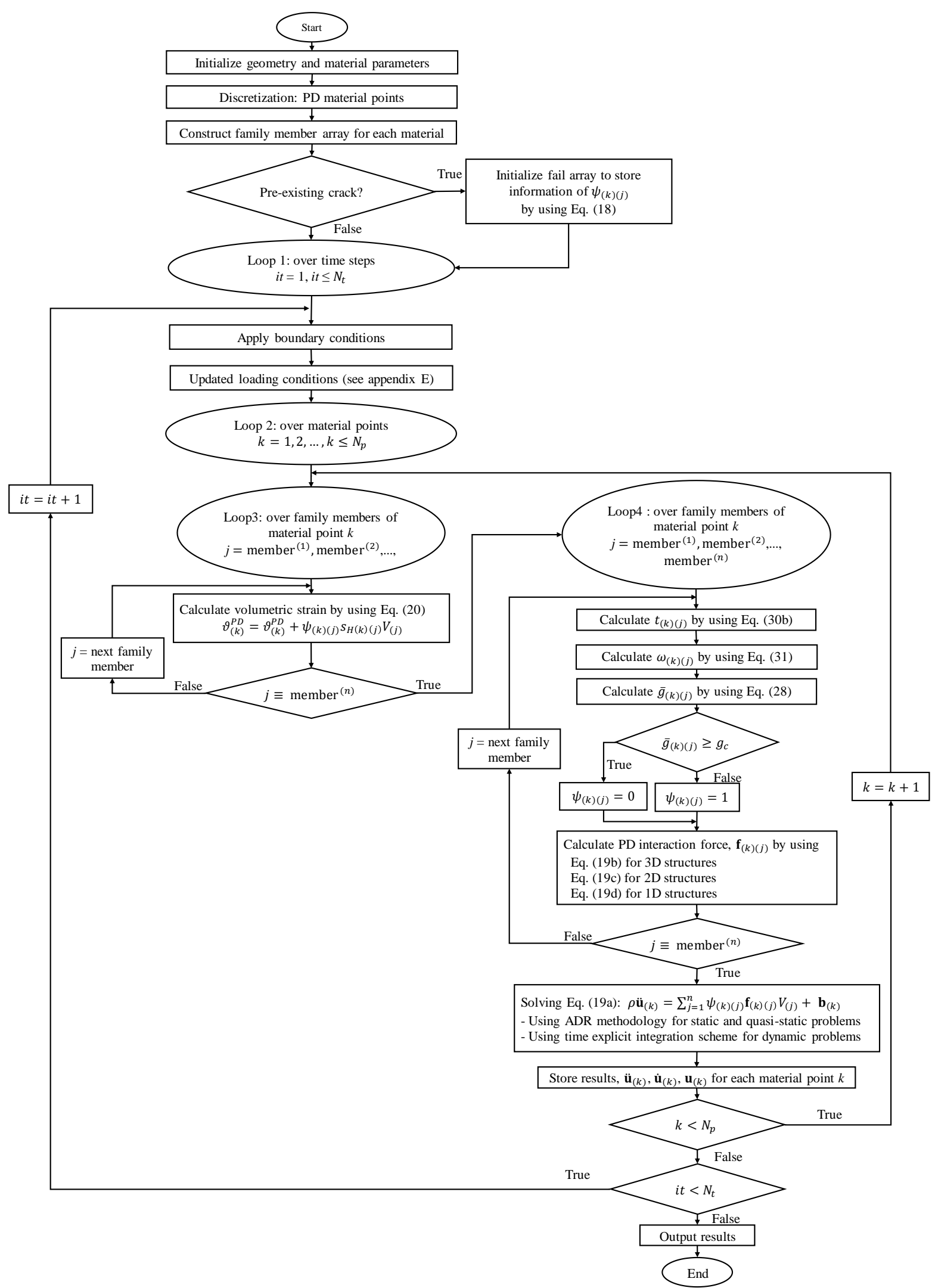

Fig. 7. Numerical procedure (ADR: Adaptive Dynamic Relaxation method $[60,61]$ )

\section{Numerical results}

In this section, first, the developed nonlinear PD model is verified by considering various examples of 1D, 2D, and 3D structures. For verification purposes, the proposed PD model is 
compared to finite element analysis (FEA) solutions. The explicit time integration is used in PD solution by using adaptive relaxation methodology [60, 61]. In PD solution, the horizon size $\delta_{H}=3.015 \Delta x$ is used. The FEA solutions are conducted by using ANSYS commercial software with LINK180 element for 1D bar, PLANE182 element for 2D plate and SOLID185 element for 3D structures. Next, damage predictions on a steel plate subjected to dynamic loading, an L-shape plate subjected to large deformations, and on a concrete beam in three points bending test are presented.

6.1. Large deformations in a $1 \mathrm{D}$ structure

In order to verify the proposed PD model for one dimension, a bar with a cross-sectional area $A=0.1 \times 0.1 \mathrm{~m}^{2}$ with length of $L=1 \mathrm{~m}$ subjected to axial loading is investigated as shown in Fig. 8. The bar is made of steel with Young's modulus $E=2 \times 10^{11} \mathrm{~N} / \mathrm{m}^{2}$. The bar is subjected to two different loading conditions which are tensile load of $F_{x}=5 \times 10^{8} \mathrm{~N}$ and compressive load of $F_{x}=-5 \times 10^{8} \mathrm{~N}$.

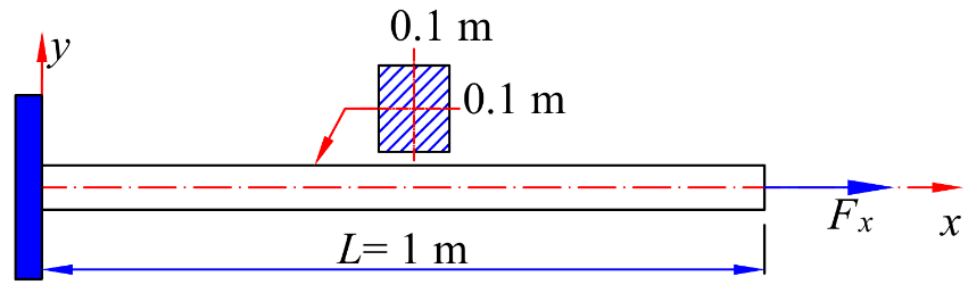

(a)

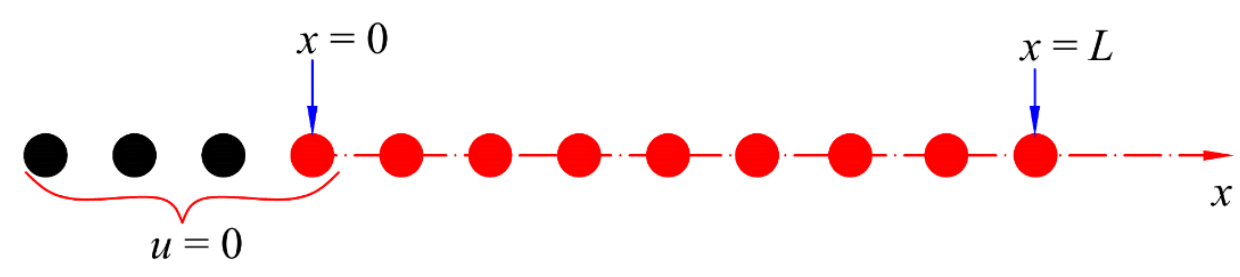

(b)

Fig. 8. Bar subjected to axial loading (a) geometry, (b) PD model discretization

In the peridynamic model, the bar is discretized with uniform 100 integration points. In order to implement the fixed end, three fictitious points [23, 62] are added as shown in Fig. 8(b) and displacements of these fictitious points and a material point located at $x=0$ are set equal to zero. The red points represent the material points in the real region, on the other hand, black points represent the material points in the fictitious region as shown in Fig. 8(b). In the FEA model, the bar is discretized with 100 elements by using link 180 element.

In both loading conditions, the constant body force $b_{x}=F_{x} / V$ is applied to the material point located at $x=L$. Details of applying loading conditions are presented in Appendix E.1.

Fig. 9 shows the displacement variations along the bar. As can be seen from Fig. 9(a), under tensile loading, the displacement of the bar in nonlinear cases is much larger than in the linear case. On the other hand, in the compressive loading condition, the nonlinear cases provide much smaller displacement values than those captured in linear case as shown in Fig. 9(b). It can be observed that results captured in nonlinear PD analysis match very well with the results in nonlinear FEA. 


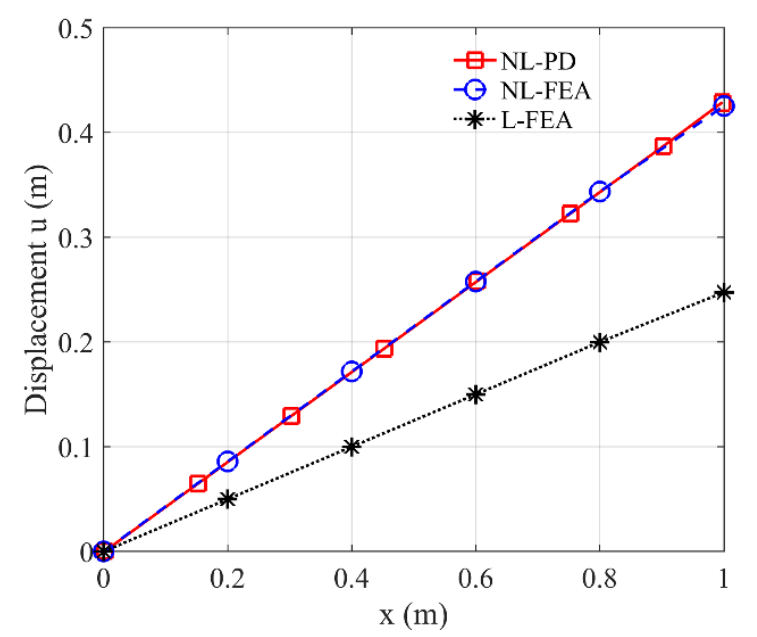

(a)

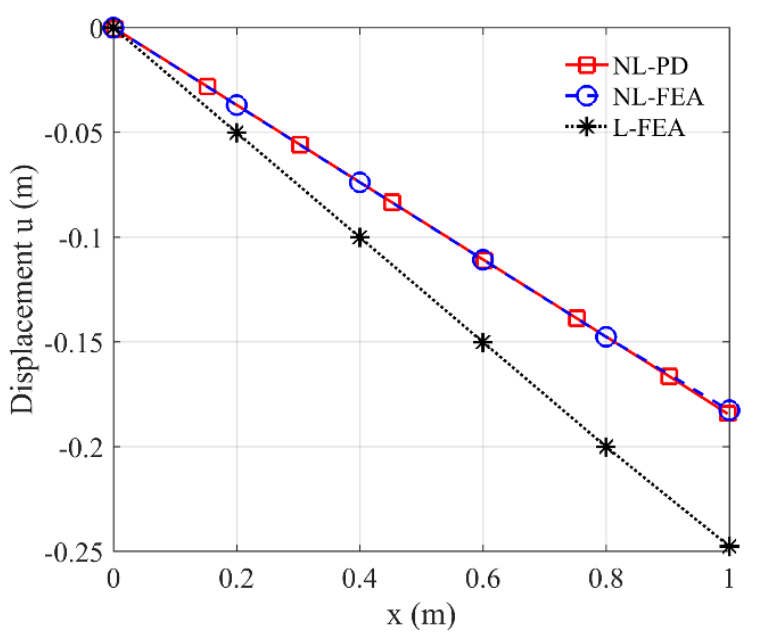

(b)

Fig. 9. Displacements of material points along the bar subjected to axial load: (a)

$$
F_{x}=5 \times 10^{8} \mathrm{~N} \text {, (b) } F_{x}=-5 \times 10^{8} \mathrm{~N} \text { (L: linear, NL: nonlinear) }
$$

In order to further verify the developed 1D nonlinear PD model, the bar described in Fig. 8 is further investigated by applying various tensile forces. Fig. 10 shows the displacement variation of the material point located at $x=1 \mathrm{~m}$. As can be seen from the figure, when the applied force is large, deformations observed in nonlinear and linear analyses have significant differences, and the results captured by the developed nonlinear PD model match very well with nonlinear FEA results.

Table. 1 shows the comparison between nonlinear PD and nonlinear FEA displacement results at $x=0.5 \mathrm{~m}$. The relative error between the two results is calculated as

$\operatorname{Error}(\%)=\left|\frac{u^{N L-P D}-u^{N L-F E A}}{u^{N L-F E A}}\right| \times 100$

As it can be seen from Table. 1, the relative errors between nonlinear PD and nonlinear FEA results are less than $0.5 \%$ for all loading cases. Therefore, it can be concluded that the developed PD model for large deformation analysis of one-dimensional structure is verified.

Table. 1. Comparison between nonlinear PD and nonlinear FEA results

\begin{tabular}{|c|c|c|c|}
\hline$F_{x}(\mathrm{~N})$ & $u^{N L-F E A}(\mathrm{~m})$ & $u^{N L-P D}(\mathrm{~m})$ & Error $(\%)$ \\
\hline $1.0 \mathrm{E}+07$ & 0.00252 & 0.00253 & $0.335 \%$ \\
\hline $5.0 \mathrm{E}+07$ & 0.01299 & 0.01303 & $0.331 \%$ \\
\hline $1.0 \mathrm{E}+08$ & 0.02706 & 0.02715 & $0.326 \%$ \\
\hline $2.0 \mathrm{E}+08$ & 0.05916 & 0.05935 & $0.316 \%$ \\
\hline $3.0 \mathrm{E}+08$ & 0.09829 & 0.09859 & $0.310 \%$ \\
\hline $4.0 \mathrm{E}+08$ & 0.14785 & 0.14833 & $0.323 \%$ \\
\hline $5.0 \mathrm{E}+08$ & 0.21442 & 0.21529 & $0.406 \%$ \\
\hline
\end{tabular}




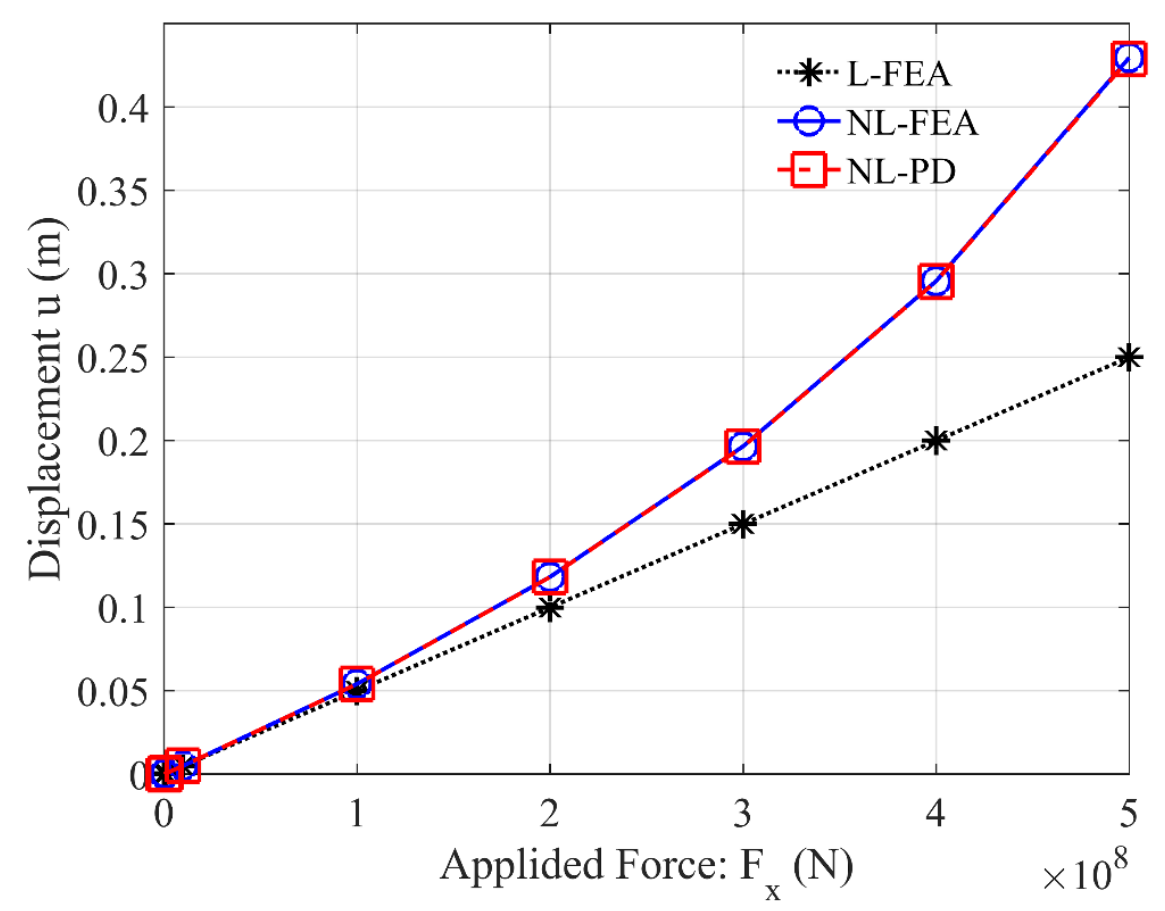

Fig. 10. Variation of displacement of the material point located at $x=1 \mathrm{~m}$ with respect to applied forces (L: linear, NL: nonlinear)

\subsection{Large deformations in 2D structures}

In order to verify the developed 2D nonlinear PD model, a square plate with $L=W=1 \mathrm{~m}$ and thickness of $h=0.01 \mathrm{~m}$ is investigated as shown in Fig. 11. The plate is made of steel with Young's modulus $E=2 \times 10^{11} \mathrm{~N} / \mathrm{m}^{2}$ and Poisson's ratio $v=0.27$. The plate is fixed on the left edge. The plate is investigated in two different loading conditions as shown in Figs. 11 and 18. In each loading condition, the plate is investigated for both plane strain and plane stress conditions.

In order to apply boundary conditions on the left edge, three fictitious layers of material points are added in the discretized model in PD as shown in Fig. 11(b) and all degrees of freedom of these fictitious points and material points located at $x=0$ are set equal to zero. The plate is discretized with $100 \times 100$ in both PD and FEA models.

\subsubsection{Plate subjected to tensile loading}

The plate is subjected to constant pressure, $p=5 \times 10^{10} \mathrm{~N} / \mathrm{m}^{2}$ normal to the surface on the right edge of the plate as shown in Fig. 11(a). In the PD model, the loading condition is applied to the material points located at $x=L$ by converting the constant pressure to body forces. Details of the implementation of loading conditions are presented in Appendix E.2. 


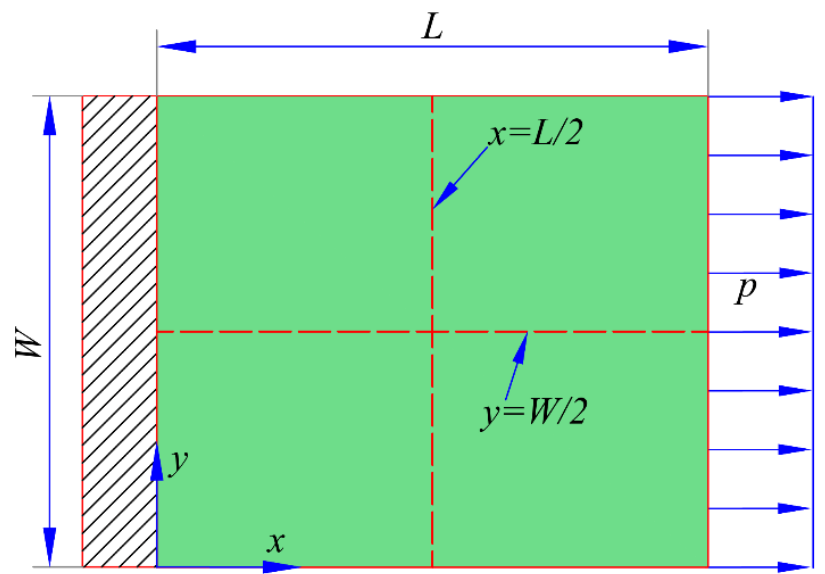

(a)

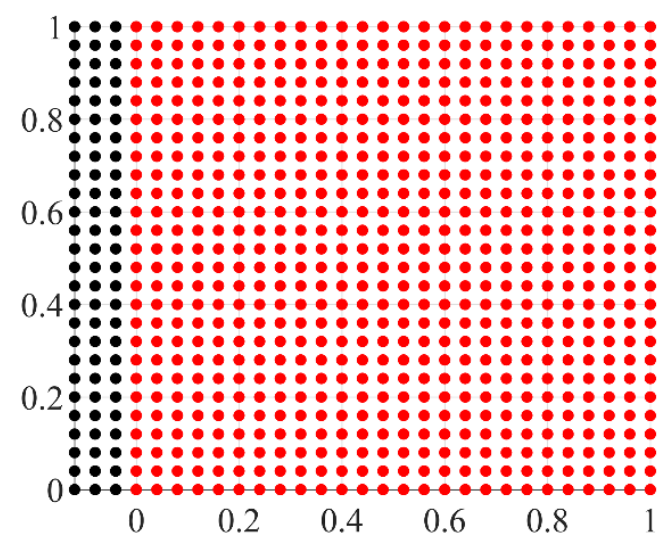

(b)

Fig. 11. Plate is subjected to tensional pressure (a) geometry, (b) PD model discretization

Plane strain condition:

Figs. 12-13 present the variations of displacement components of the plate for plane strain condition. As can be seen from the figures, the PD and nonlinear FEA prediction results match very well.

Fig. 14 shows the comparison between nonlinear PD and nonlinear FEA results for the variations of displacement components along two centerlines $x=L / 2$ and $y=W / 2$. Fig. 14(a) represents the horizontal displacements at $x=L / 2$ and Fig. 14(b) represents the vertical displacements at $y=W / 2$. As can be seen from the results, PD and nonlinear FEA solutions agree very well, and there is a big difference between linear FEA and nonlinear solutions.

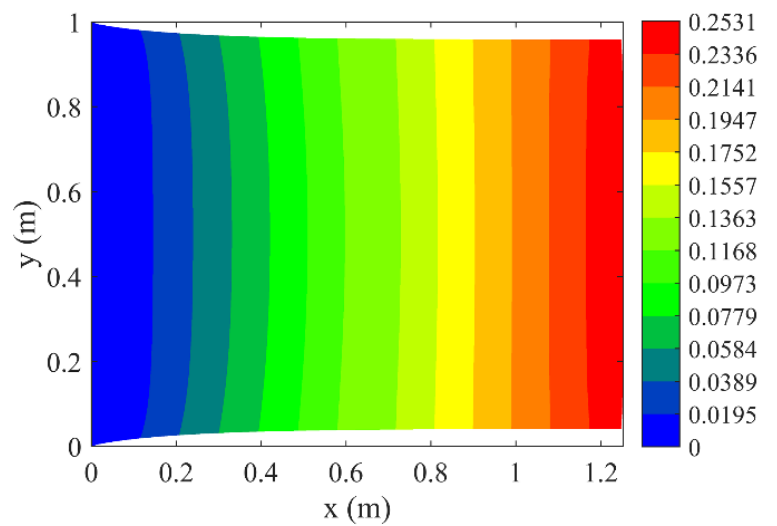

(a)

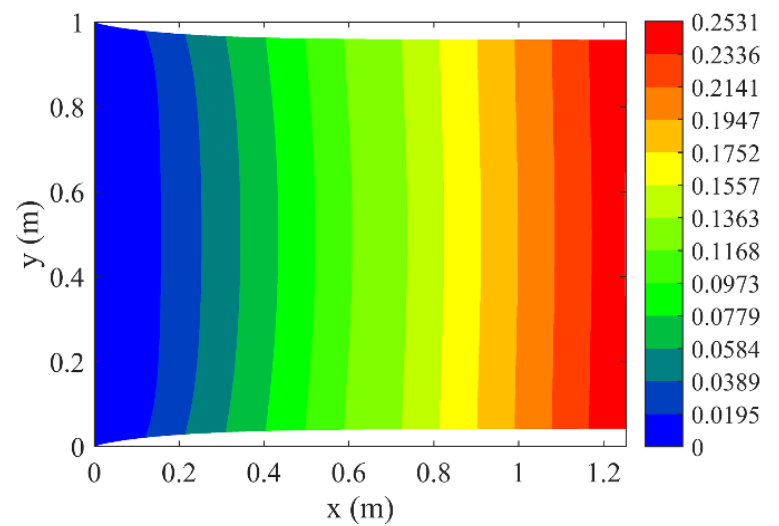

(b)

Fig. 12. Variation of horizontal displacements, $u$ in (a) nonlinear PD; (b) nonlinear FEA in deformed configuration 


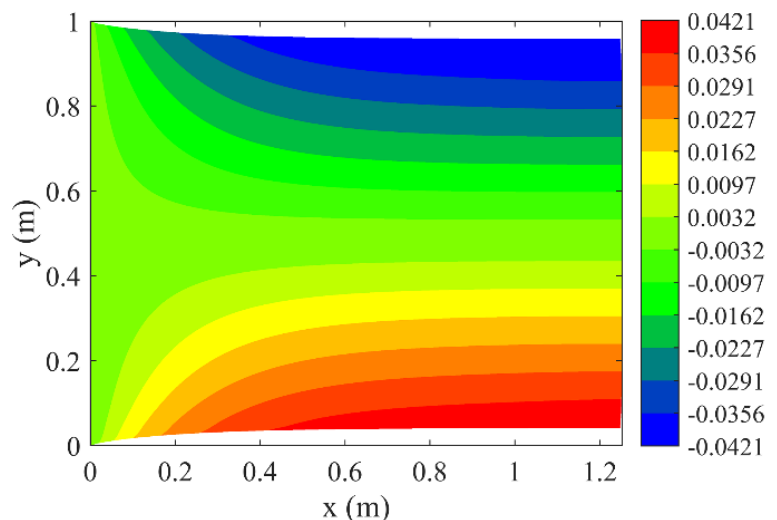

(a)

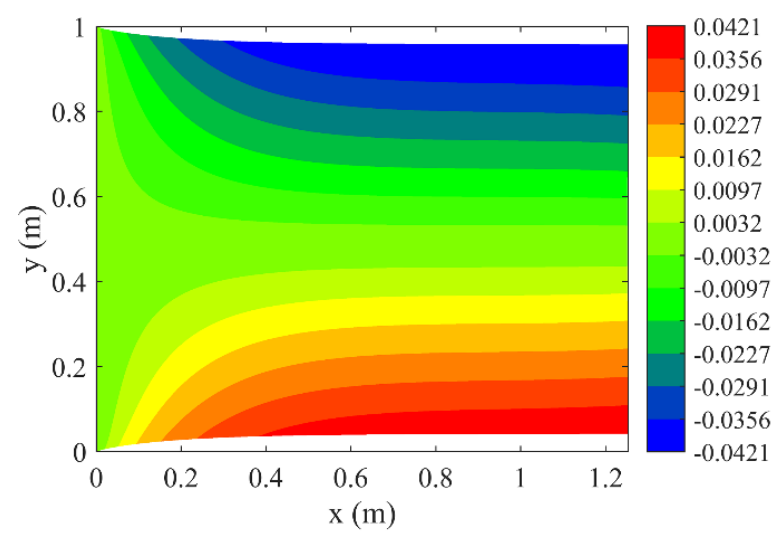

(b)

Fig. 13. Variation of vertical displacements, $v$ in (a) nonlinear PD; (b) nonlinear FEA in deformed configuration

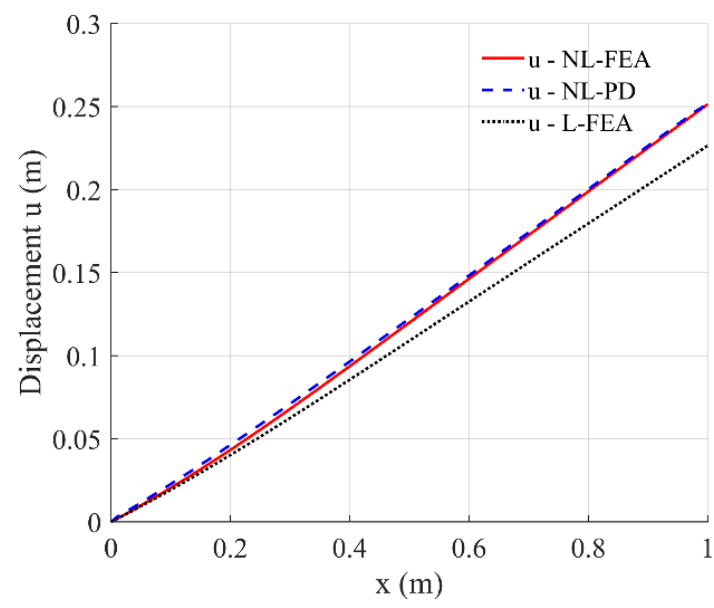

(a)

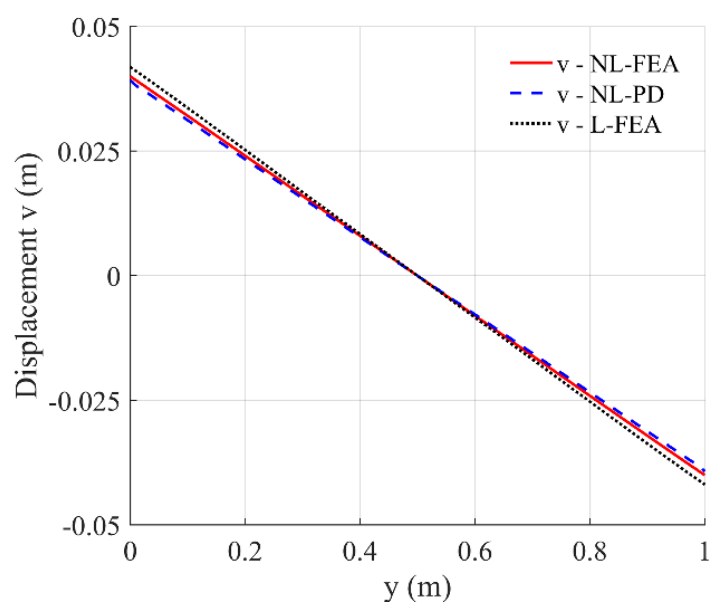

(b)

Fig. 14. Variations of displacements (a) $u$ along $y=W / 2$; (b) $v$ along $x=L / 2$ (L: linear, NL: nonlinear)

Plane stress condition:

Figs. 15-17 represent the variations of displacement components of the plate for plane stress conditions. Similar to the plane strain problem, the variations of displacement components along the center lines are investigated. It is observed that the results provided by the developed PD model have good agreement with those captured in nonlinear FEA.
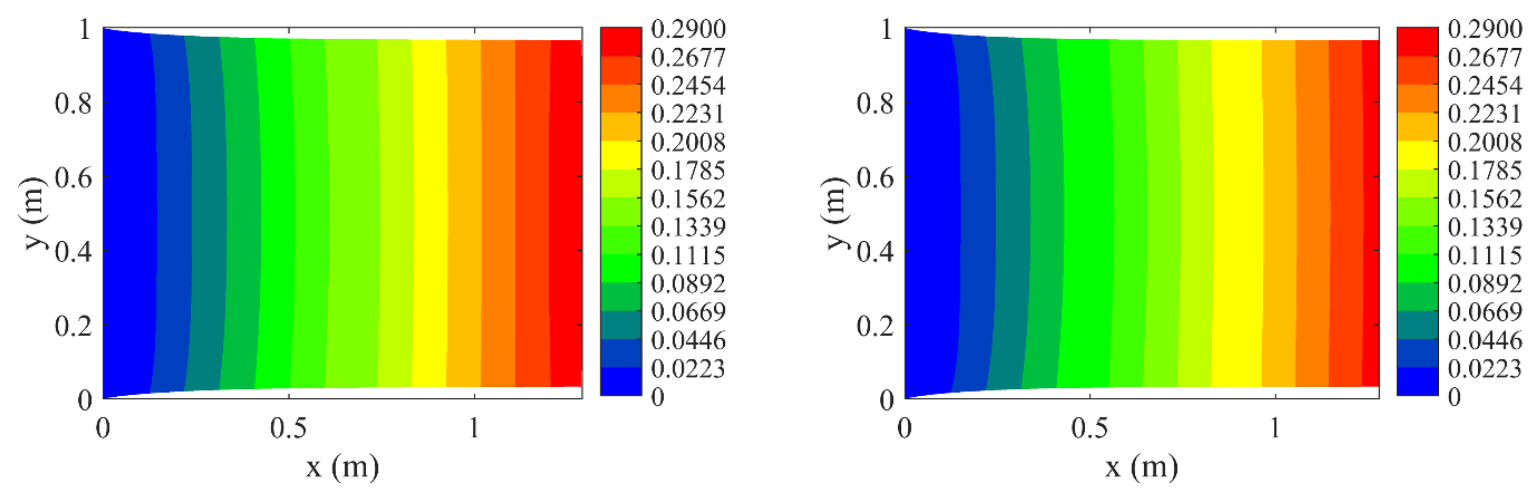
(a)

Fig. 15. Variation of horizontal displacements, $u$ in (a) nonlinear PD; (b) nonlinear FEA in deformed configuration

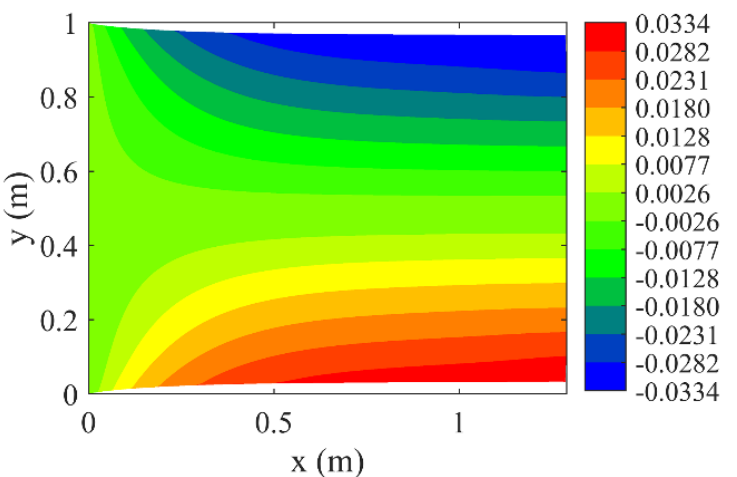

(a)

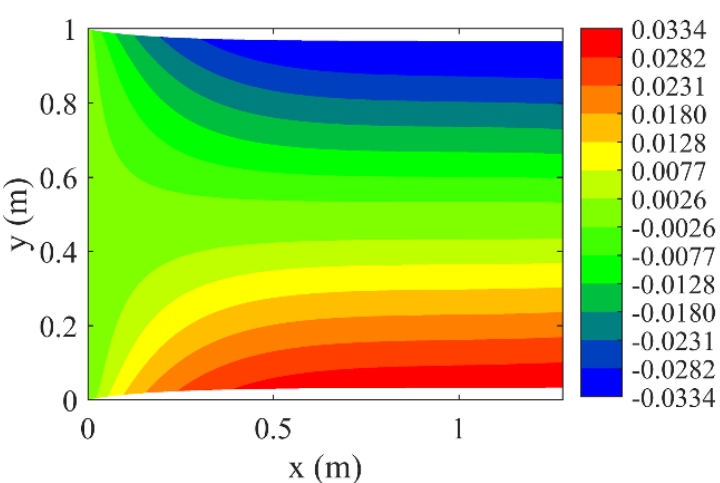

(b)

Fig. 16. Variation of vertical displacements, $v$ in (a) nonlinear PD; (b) nonlinear FEA in deformed configuration

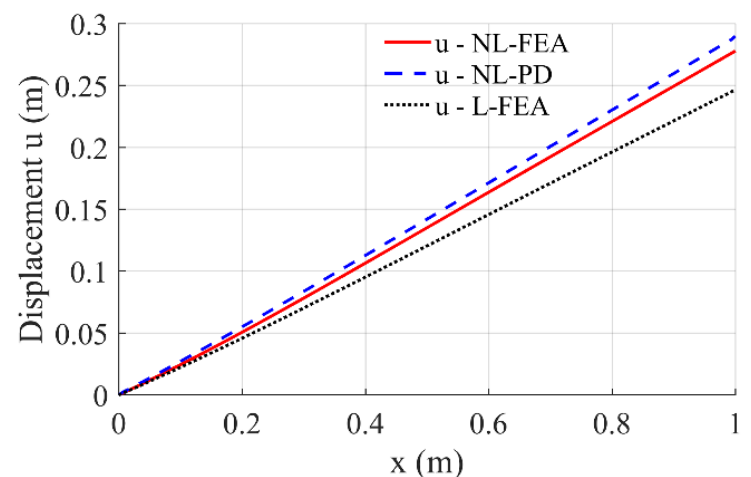

(a)

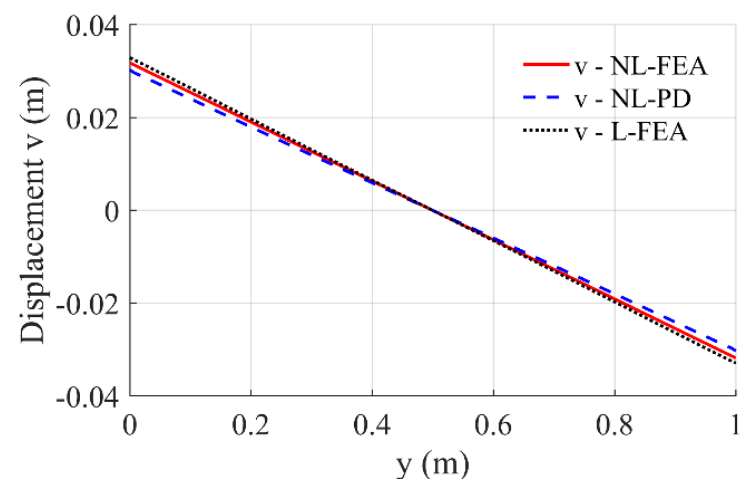

(b)

Fig. 17. Variations of displacements (a) $u$ along $y=W / 2$; (b) $v$ along $x=L / 2$ (L: linear, NL: nonlinear)

\subsubsection{Plate subjected to shear loading}

In order to further verify the developed PD model, the plate is considered in a shear loading condition. The plate is subjected to uniform loading $p_{2}=1.3 \times 10^{10} \mathrm{~N} / \mathrm{m}^{2}$ normal to the surface on the top edge as shown in Fig. 18. The plate is also investigated for both plane strain and plane stress conditions.

In the PD model, the loading condition is applied to the material points located at $y=W$ by converting the constant pressure to body forces. Details of the implementation of loading conditions are presented in Appendix E.2. 


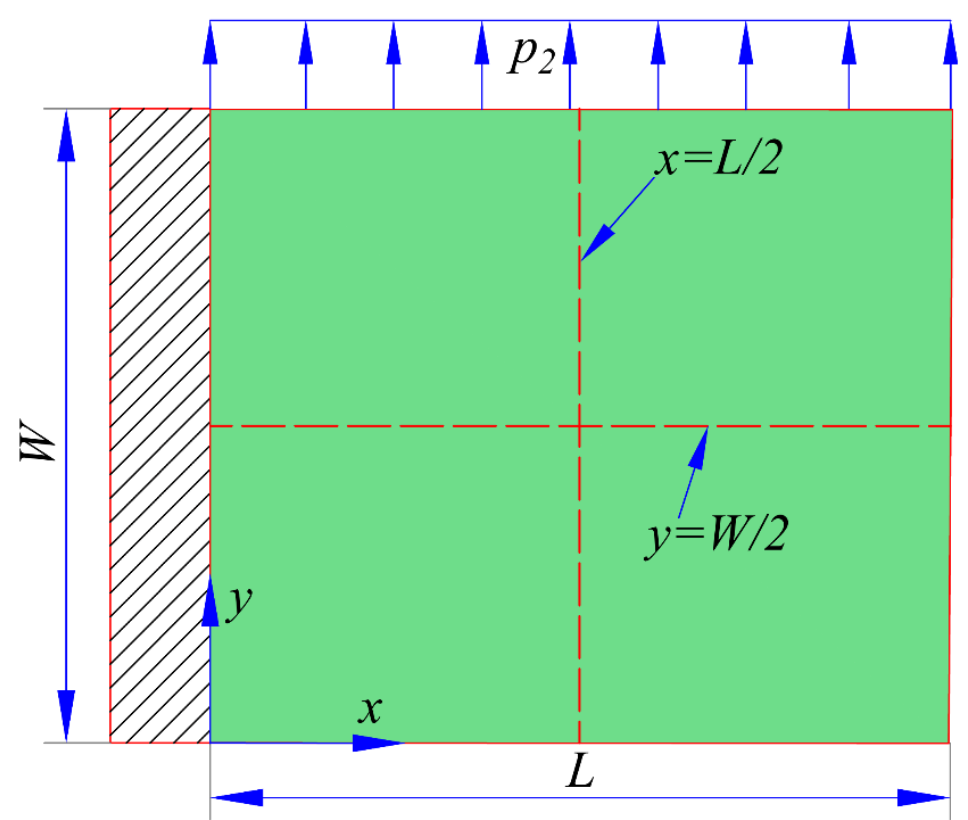

Fig. 18. Plate is subjected to pressure on top

Plane strain condition

Figs. 19-22 present the results for plane strain condition. As can be seen from Fig. 19 and Fig. 20, the variations of displacements $u$ and $v$ captured in PD and FEA solutions have a very good agreement. Moreover, as presented in Fig. 21 and 22, the results captured by the developed PD model agree very well with those in nonlinear FEA whereas a significant difference between linear and nonlinear results is observed.

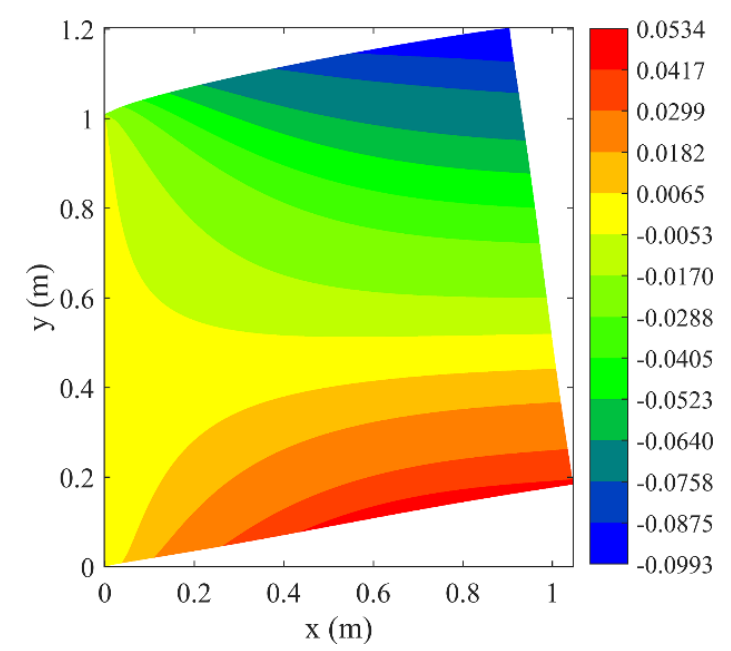

(a)

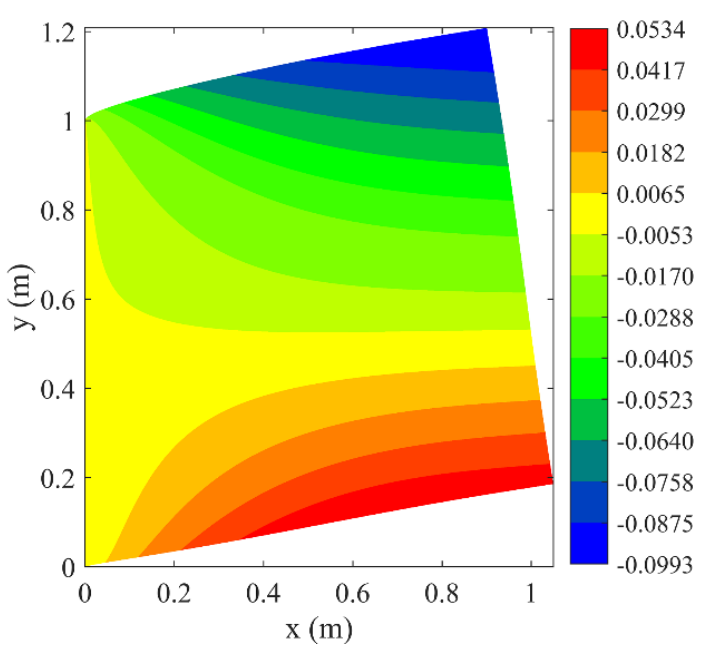

(b)

Fig. 19. Variation of horizontal displacements, $u$ in (a) nonlinear PD; (b) nonlinear FEA in deformed configuration 


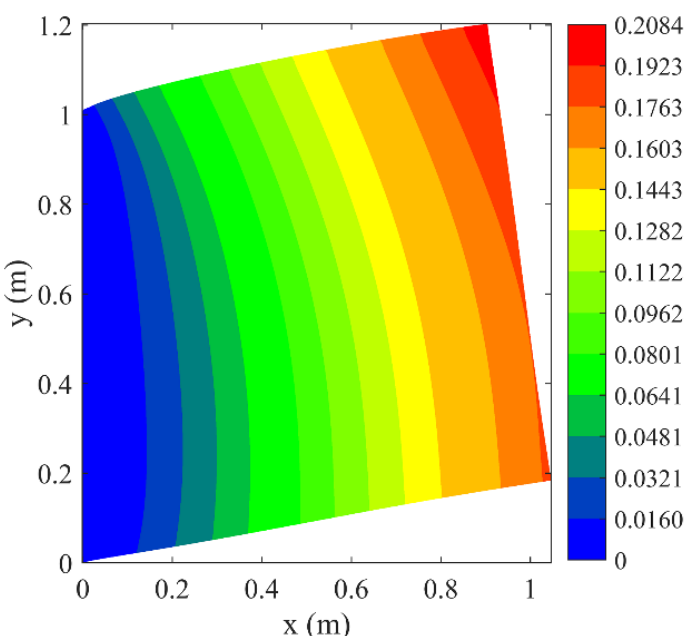

(a)

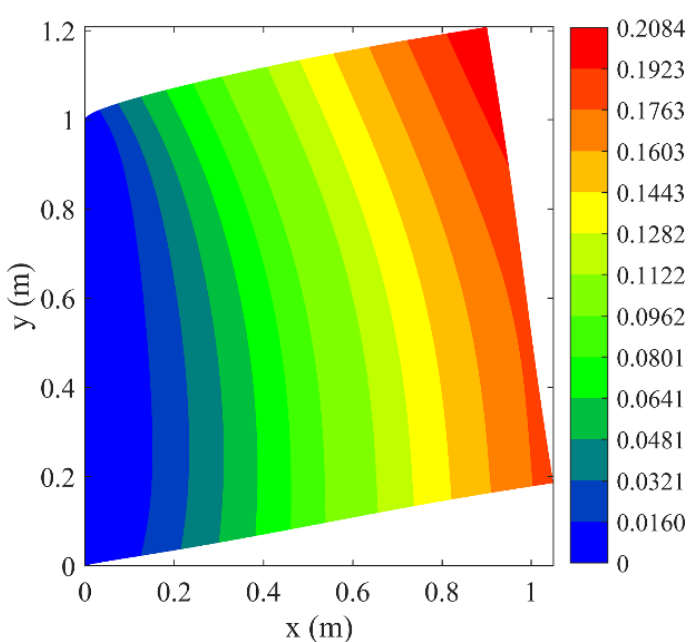

(b)

Fig. 20. Variation of vertical displacements, $v$ in (a) nonlinear PD; (b) nonlinear FEA in deformed configuration

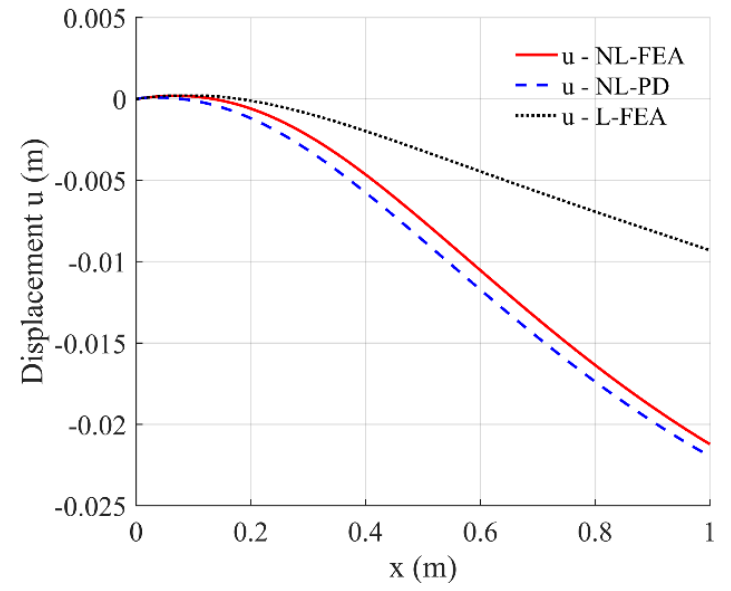

(a)

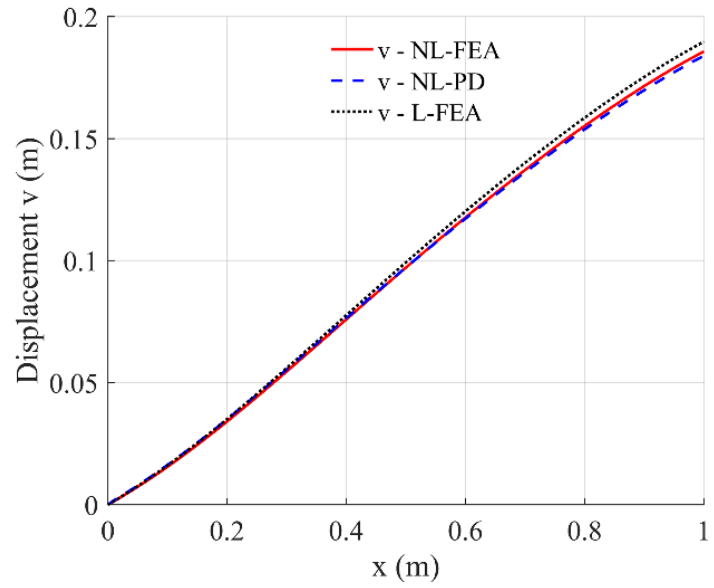

(b)

Fig. 21. Variations of displacement components (a) $u$, (b) $v$ at $y=W / 2$ (L: linear, NL: nonlinear)
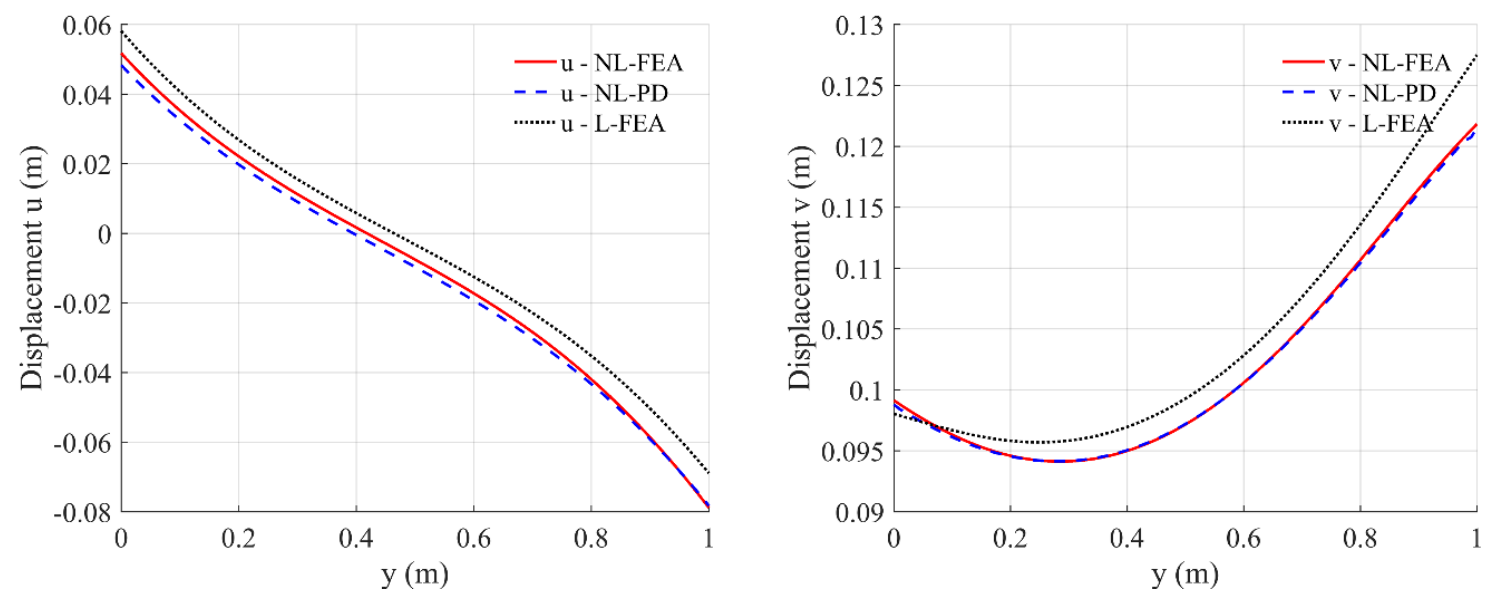
Fig. 22. Variations of displacement components (a) $u$, (b) $v$ along $x=L / 2$ (L: linear, NL: nonlinear)

\section{Plane stress condition}

Figs. 23-26 present the results for the plane stress condition. As can be seen from the figures, the results in nonlinear PD and nonlinear FEA solutions match very well. Therefore, it can be concluded that the accuracy of the developed 2D nonlinear PD model is verified.

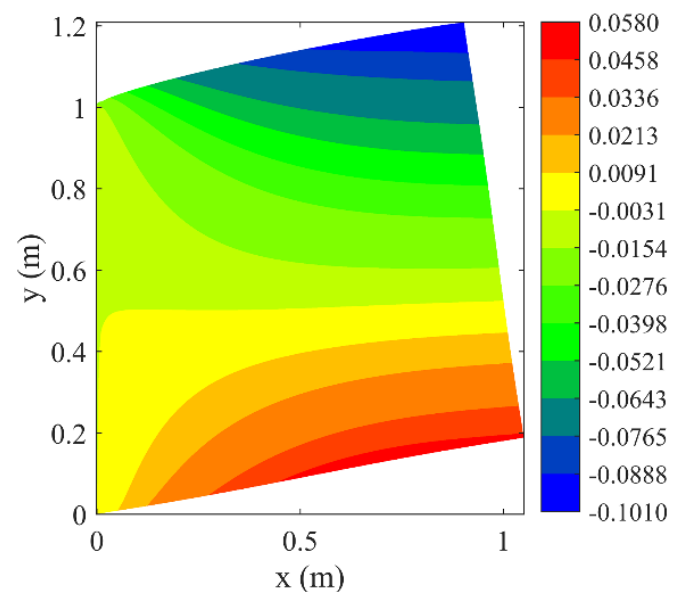

(a)

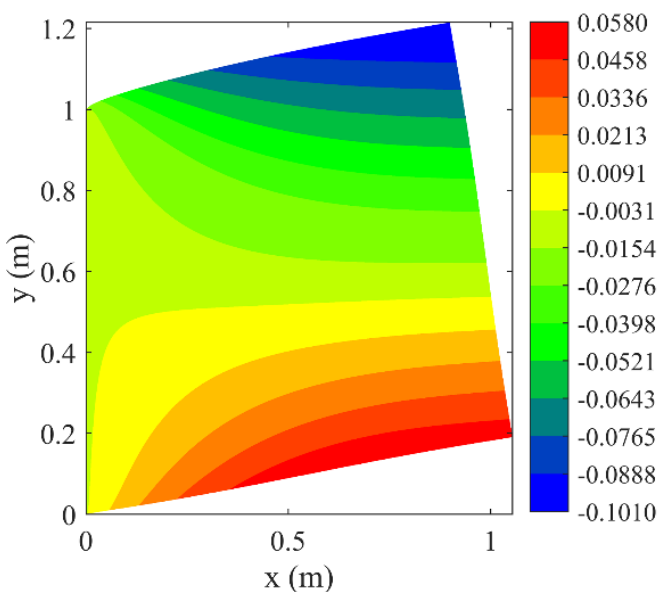

(b)

Fig. 23. Variation of horizontal displacements, $u$ in (a) nonlinear PD; (b) nonlinear FEA in deformed configuration

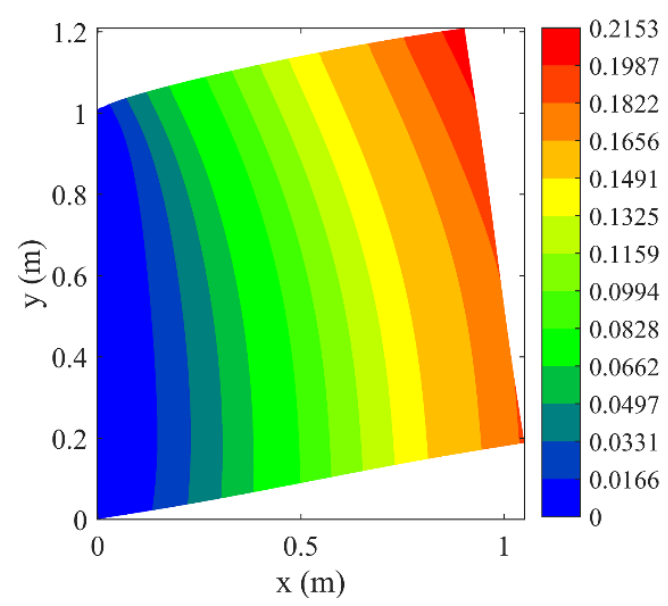

(a)

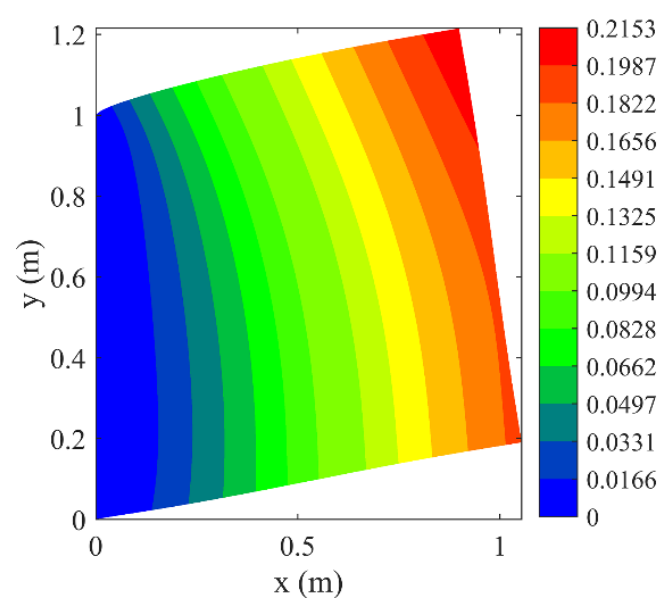

(b)

Fig. 24. Variation of vertical displacements, $v$ in (a) nonlinear PD; (b) nonlinear FEA in deformed configuration 


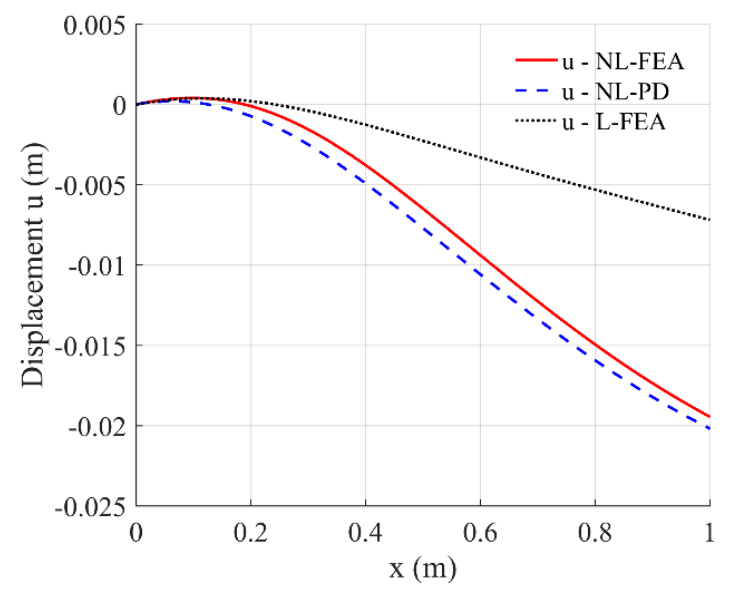

(a)

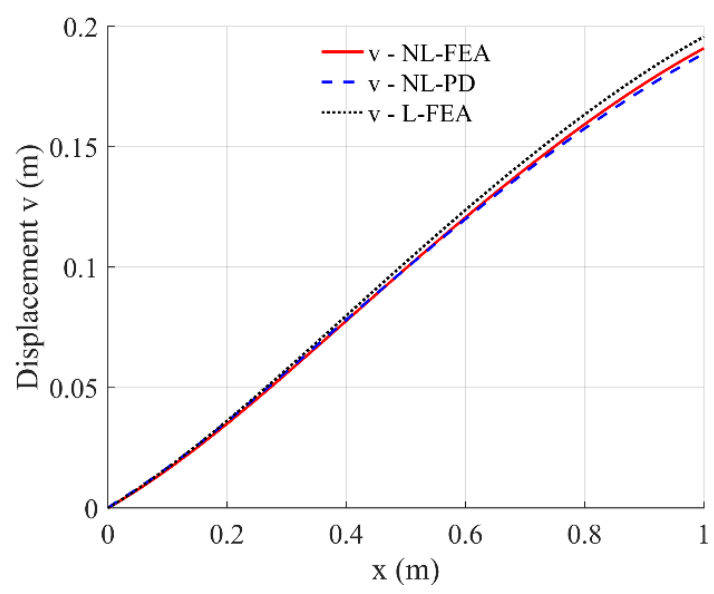

(b)

Fig. 25. Variations of displacement components (a) $u$, (b) $v$ along $y=L / 2$ (L: linear, NL: nonlinear)

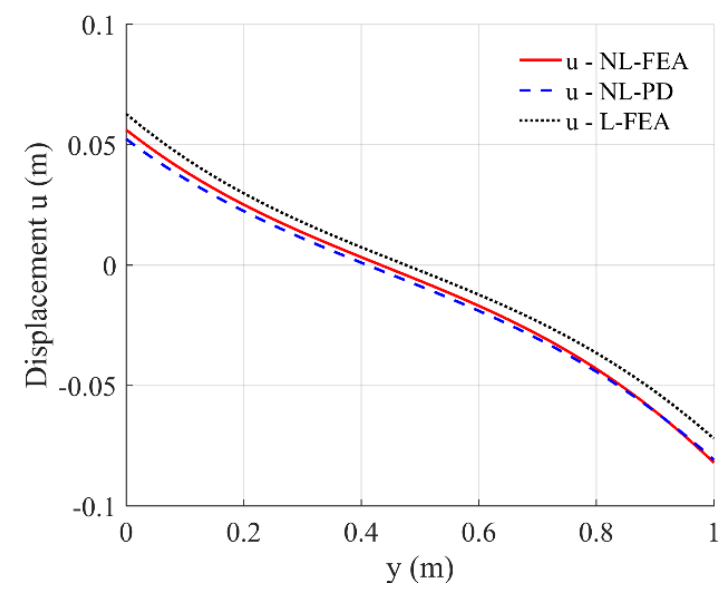

(a)

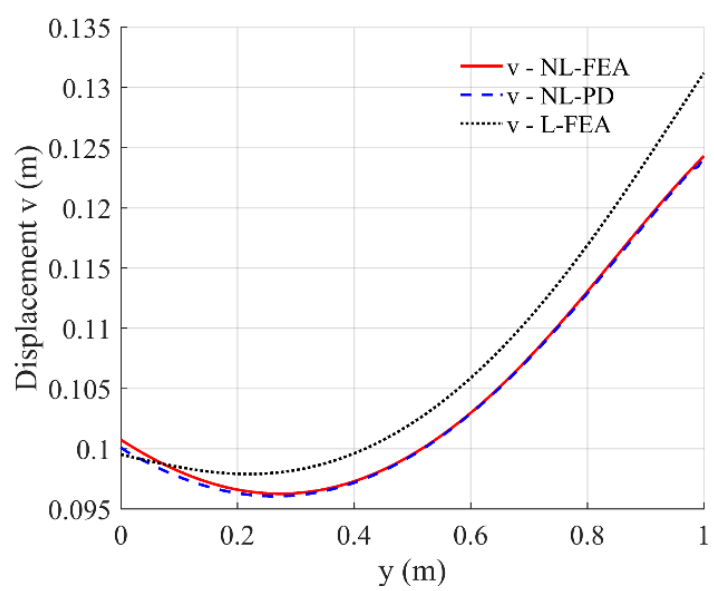

(b)

Fig. 26. Variations of displacement components (a) $u$, (b) $v$ along $x=L / 2$ (L: linear, NL: nonlinear)

For further comparison, the displacements at $(x=3 L / 4, y=3 W / 4)$ obtained from nonlinear PD and nonlinear FEA are compared as shown in Table. 2. As it can be seen from the table, the relative errors between the results are less than 5\% for all loading conditions. Therefore, the accuracy of the developed nonlinear PD model for 2D structures is verified for both plane stress and plane strain conditions.

Table. 2. Comparison of displacements for a material point located at $(x=3 L / 4, y=3 \mathrm{~W} / 4)$

\begin{tabular}{|l|l|c|c|c|c|c|c|}
\hline \multicolumn{2}{|c|}{} & $\begin{array}{c}u^{\text {NLFEA }} \\
(\mathrm{m})\end{array}$ & $\begin{array}{c}u^{\text {NLPD }} \\
(\mathrm{m})\end{array}$ & $\begin{array}{c}\text { Error } \\
(\%)\end{array}$ & $\begin{array}{c}v^{\text {NLFEA }} \\
(\mathrm{m})\end{array}$ & $\begin{array}{c}v^{\text {NLPD }} \\
(\mathrm{m})\end{array}$ & $\begin{array}{c}\text { Error } \\
(\%)\end{array}$ \\
\hline $\begin{array}{l}\text { Plate } \\
\text { subjected to } \\
\begin{array}{l}\text { tensile } \\
\text { loading }\end{array}\end{array}$ & $\begin{array}{l}\text { Plane } \\
\text { strain }\end{array}$ & 0.2531 & 0.2485 & $1.8 \%$ & -0.0421 & -0.0416 & $1.2 \%$ \\
\cline { 2 - 8 } & $\begin{array}{l}\text { Plane } \\
\text { stress }\end{array}$ & 0.2792 & 0.2862 & $2.5 \%$ & -0.0334 & -0.0334 & $0.0 \%$ \\
\hline $\begin{array}{l}\text { Plate } \\
\text { subjected to } \\
\text { shear } \\
\text { loading }\end{array}$ & $\begin{array}{l}\text { Plane } \\
\text { strain }\end{array}$ & -0.0993 & -0.0959 & $3.4 \%$ & 0.2084 & 0.2029 & $2.6 \%$ \\
\cline { 2 - 8 } & $\begin{array}{l}\text { Plane } \\
\text { stress }\end{array}$ & -0.101 & -0.0969 & $4.0 \%$ & 0.2153 & 0.209 & $2.9 \%$ \\
\hline
\end{tabular}


6.3. Large deformations in 3D structures

In order to verify the proposed PD model for 3D structures, a 3D beam subjected to constant shear force is investigated as shown in Fig. 27. The dimensions of the beam are $L \times B \times H=1 \times 0.1 \times 0.1 \mathrm{~m}^{3}$ and it is made of steel with Young's modulus $E=2.06 \times 10^{11} \mathrm{~N} / \mathrm{m}^{2}$ and Poisson's ratio $v=0.3$. The structure is subjected to a distributed force $f_{z}=-1 \times 10^{8} \mathrm{~N} / \mathrm{m}$ at the right end as shown in Fig. 27.
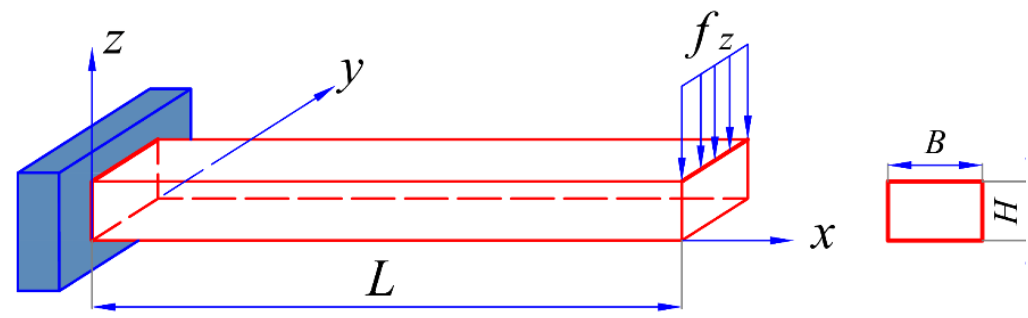

(a)

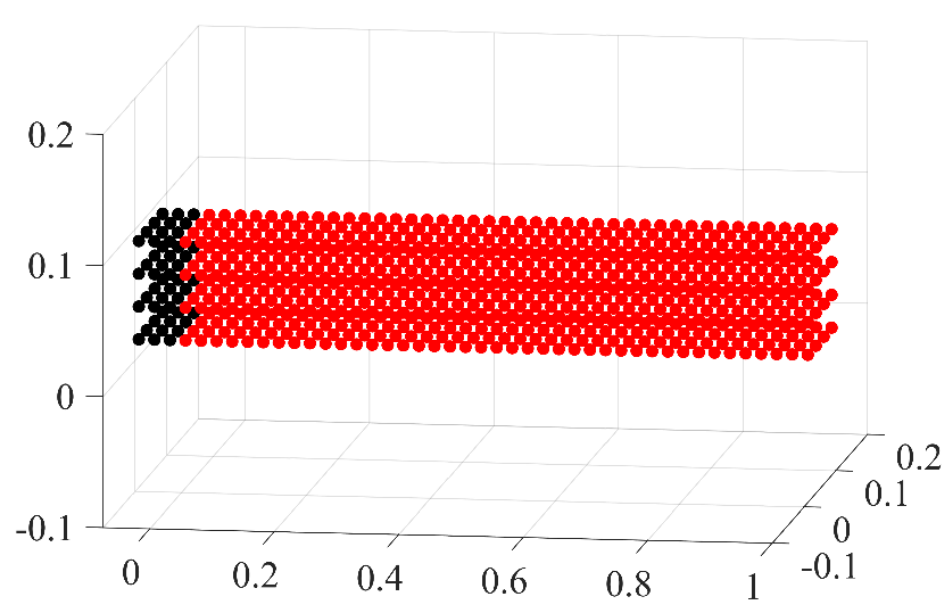

(b)

Fig. 27. 3D beam subjected to static loading (a) geometry, (b) PD model discretization

In the peridynamic model, the $3 \mathrm{D}$ beam is discretized with uniform $101 \times 10 \times 10$ material points. In FEA model, the same mesh size is used.

In order to apply boundary conditions, three fictitious layers of material points [23, 62] are added as shown in Fig. 27(b) and displacements of these fictitious points and material points located at $x=0$ are set equal to zero. As shown in Fig. 27(b), red points represent the material points in the real region, on the other hand, black points represent the material points in the fictitious region.

In the PD model, the loading condition is applied to the material points located at $x=L, 0 \leq y \leq B, z=H$ as body forces. The details of applying loading conditions in PD simulation are presented in Appendix E.3.

Figs. 28-30 show the displacement variations along the beam. The PD predictions are compared with nonlinear and linear FEA results. As shown in Fig. 28 for the variation of displacement $u$, both nonlinear PD model and nonlinear FEA provide similar results, meanwhile, the linear FEA results are completely different. The maximum displacement captured by linear FEA is almost 8 times larger than nonlinear PD and FEA solutions. Moreover, the variation of 
displacement $v$ along the beam captured by linear FEA is completely different from the nonlinear PD and FEA results as shown in Fig. 29. In Fig. 30, it is observed that the maximum displacement $w$ of the beam captured by linear FEA solution is two times larger than the maximum deflection captured by nonlinear solutions. As can be seen from the results, the developed 3D nonlinear PD model and nonlinear FEA solution show very good agreement for all displacement components.

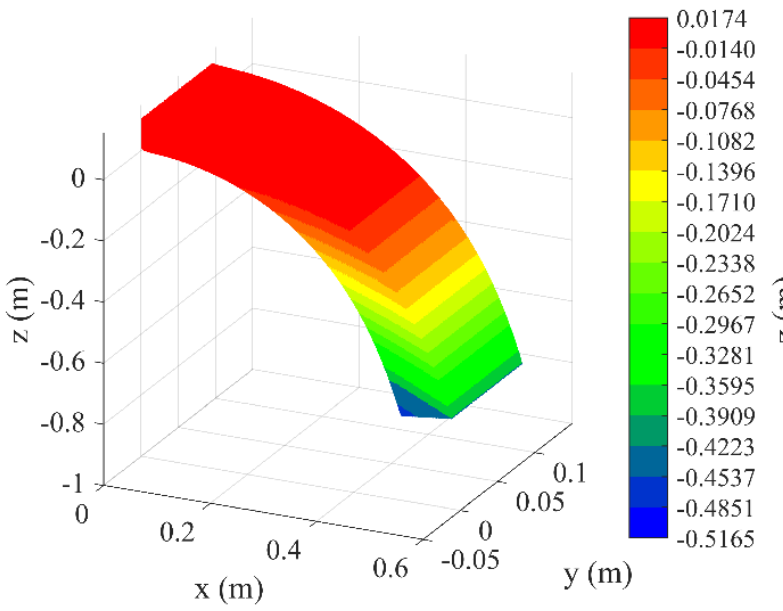

(a)

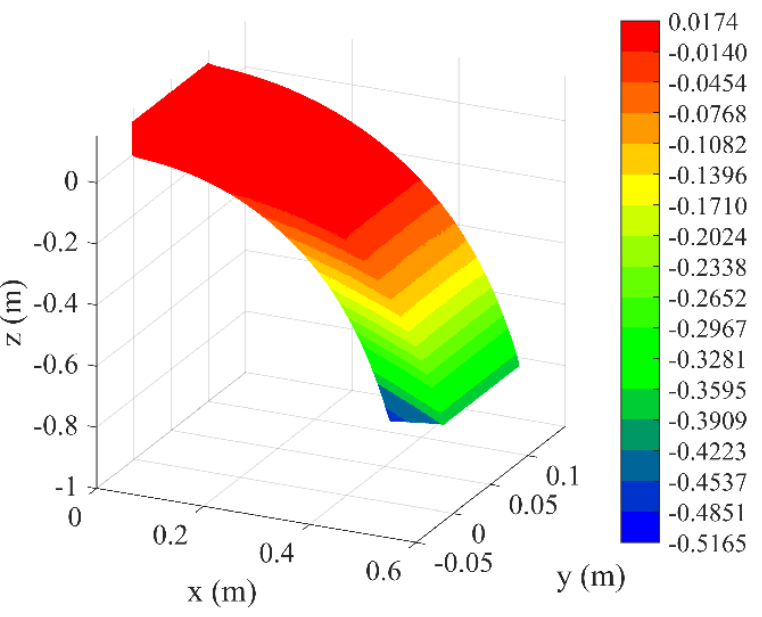

(b)

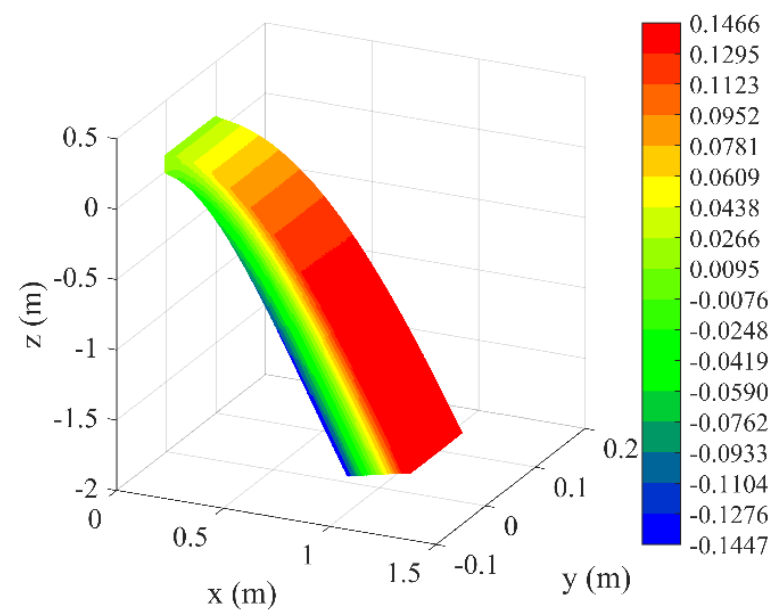

(c)

Fig. 28. Variations of displacement component $u$ in (a) nonlinear PD; (b) nonlinear FEA; (c) linear FEA results in deformed configuration 


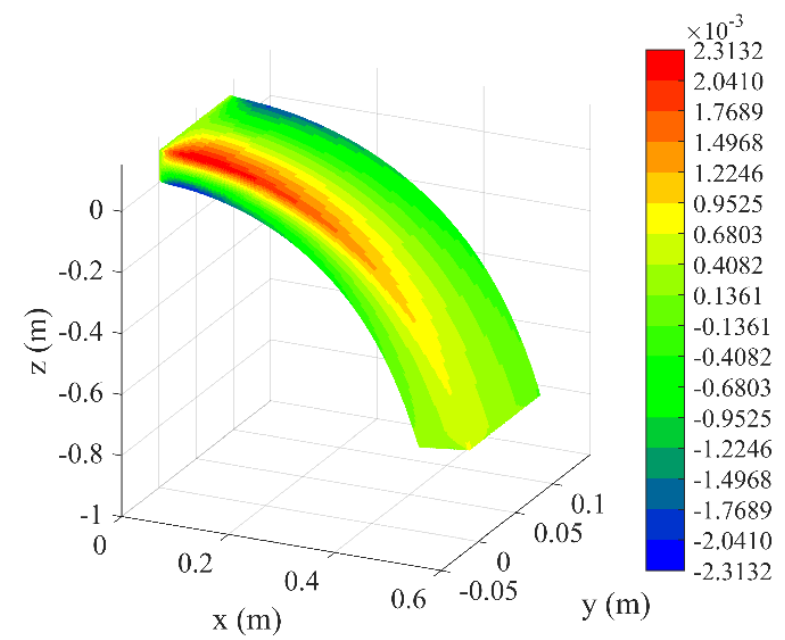

(a)

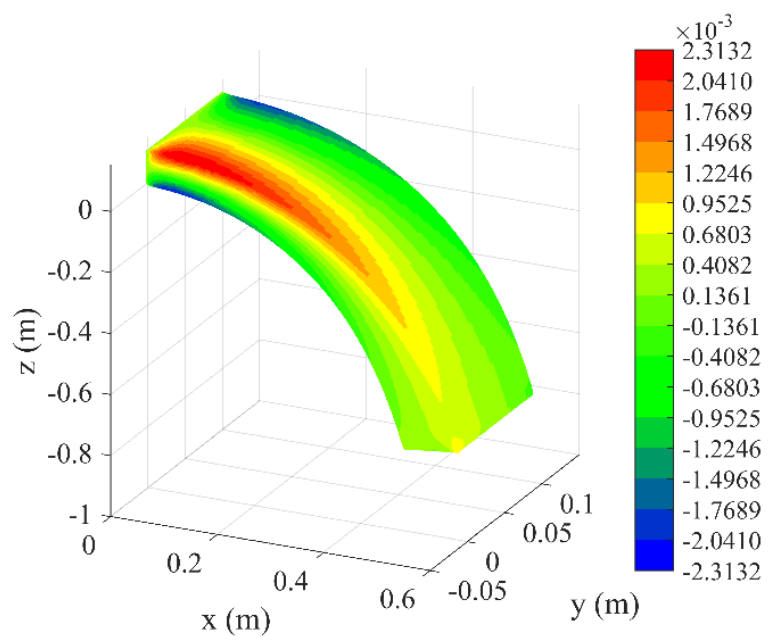

(b)

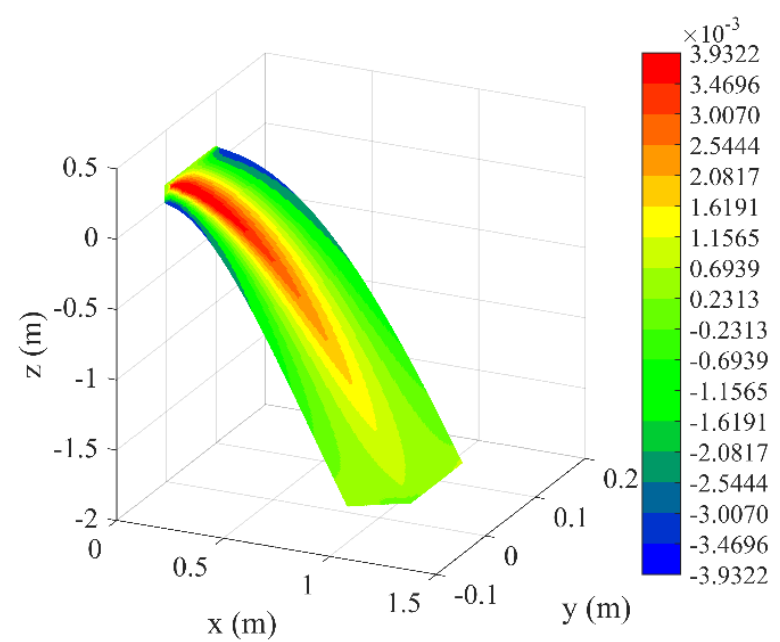

(c)

Fig. 29. Variations of displacement component $v$ in (a) nonlinear PD; (b) nonlinear FEA; (c) linear FEA results in deformed configuration

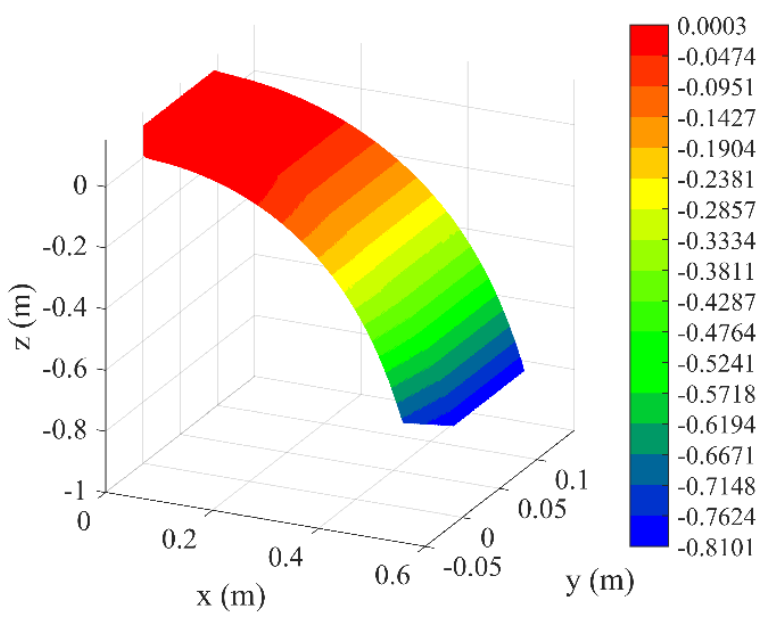

(a)

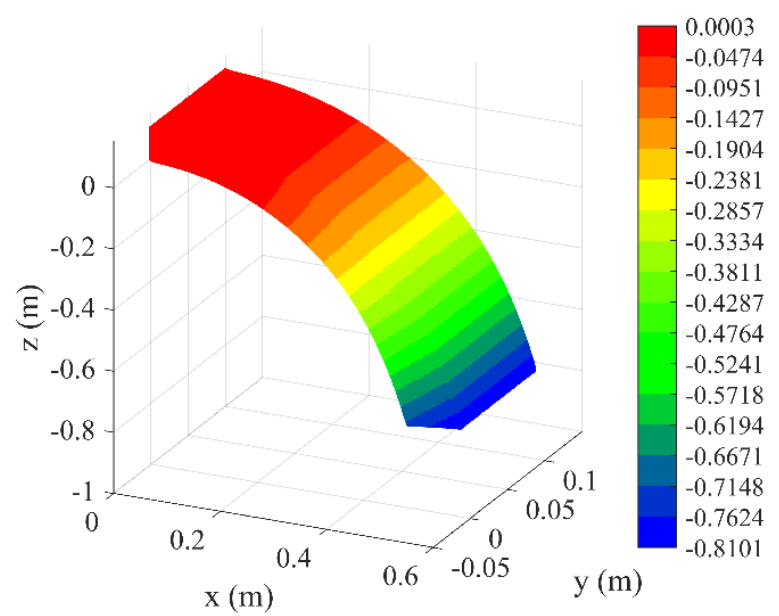

(b) 


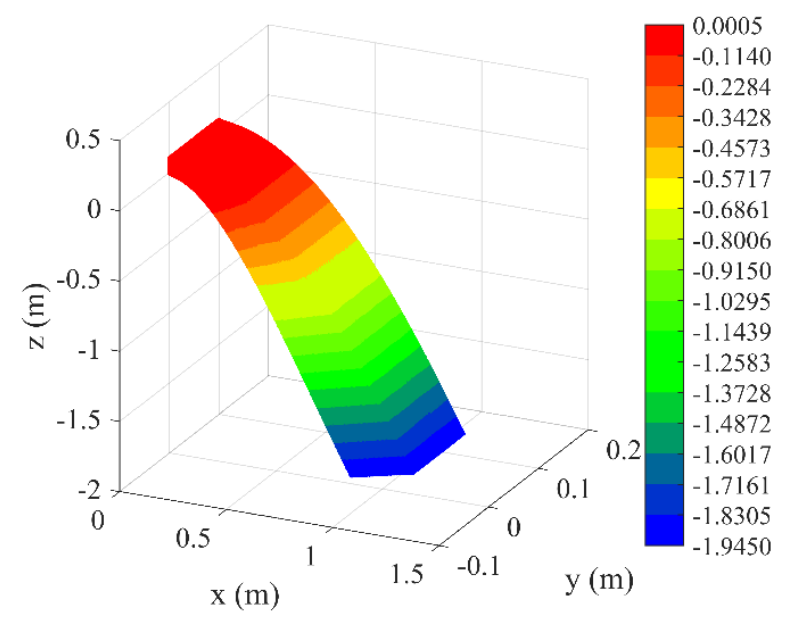

(c)

Fig. 30. Variations of displacement component $w$ in (a) nonlinear PD; (b) nonlinear FEA; (c) linear FEA results in the deformed configuration

Similar to previous examples, for further comparison, the nonlinear PD and nonlinear FEA displacements at $(x=3 L / 4, y=3 B / 4, z=3 H / 4)$ are compared as shown in Table. 3 . It is found that the relative errors for all displacement components are smaller than $1 \%$. Therefore, it can be concluded that the accuracy of the developed 3D nonlinear PD model is verified.

Table. 3. Comparison of displacements for a material point located at $(x=3 L / 4, y=3 W / 4)$

\begin{tabular}{|c|c|c|c|}
\hline Displacement: $u(\mathrm{~m})$ & $u^{\text {NLFEA }}$ & $u^{\text {NLPD }}$ & Error $(\%)$ \\
\cline { 2 - 4 } & -0.248143 & -0.248328 & $0.07 \%$ \\
\hline \multirow{2}{*}{ Displacement: $v(\mathrm{~m})$} & $v^{\text {NLFEA }}$ & $v^{\text {NLPD }}$ & Error $(\%)$ \\
\cline { 2 - 4 } & -0.000131 & -0.000130 & $0.70 \%$ \\
\hline \multirow{2}{*}{ Displacement: $w(\mathrm{~m})$} & $w^{\text {NLFEA }}$ & $w^{\text {NLPD }}$ & Error $(\%)$ \\
\cline { 2 - 4 } & -0.534627 & -0.539694 & $0.95 \%$ \\
\hline
\end{tabular}

6.4. Damage prediction in 2D plate

After verifying the accuracy of the developed nonlinear PD model for 2D structures, in this section, damage on a plate is predicted. The experiment presented by Kalthoff and Winkler [56], Kalthoff $[54,55]$ for a pre-notched plate subjected to dynamic load is simulated by using the developed PD model.

Since the problem is symmetric, only the upper haft plate is modeled as shown in Fig. 31 . The plate with $L=W=0.1 \mathrm{~m}$ and thickness of $h=0.009 \mathrm{~m}$ is investigated [54]. The plate is made of steel with Young's modulus $E=2 \times 10^{11} \mathrm{~N} / \mathrm{m}^{2}$, Poisson's ratio $v=0.27$. The fracture toughness of steel is $K_{I c}=70 \times 10^{6} \mathrm{Nm}^{-3 / 2}$ [54] and the critical energy release rate is $G_{c}=2.2714 \times 10^{4} \mathrm{~J} / \mathrm{m}^{2}$.

The left edge which is under the crack surface is subjected to velocity conditions as $[41,63]$

$|\mathbf{v}|= \begin{cases}\frac{t}{t_{0}} v_{0} & \text { for } t \leq t_{0} \\ v_{0} & \text { for } t>t_{0}\end{cases}$

with $v_{0}=16.5 \mathrm{~m} / \mathrm{s}, t_{0}=1 \mu \mathrm{s}$. 
The plate is considered in the plane stress condition and it is discretized into $200 \times 200$ material points. The horizon size is $\delta_{H}=3.015 \Delta x$. The problem is simulated using dynamic explicit time integration scheme with time step $0.01 \mu \mathrm{s}$ and total simulation time of $80 \mu \mathrm{s}$.

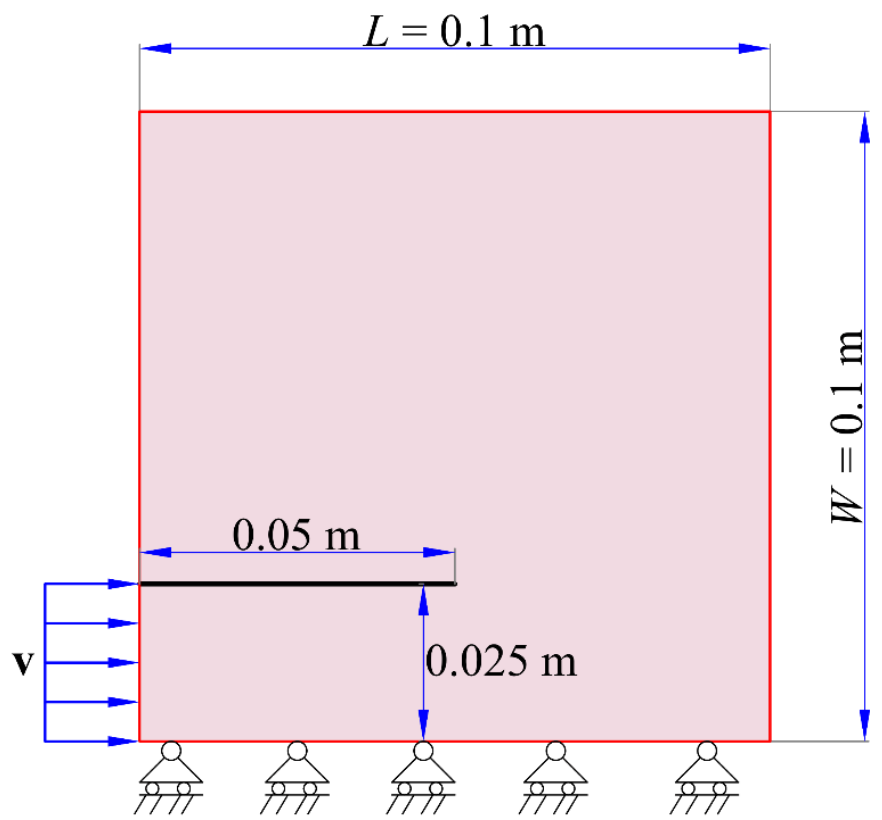

Fig. 31. The geometry and symmetrical boundary conditions for Kalthoff experiment

Figs. 32 presents the crack evolution at different times captured by nonlinear PD model with horizon size of $\delta_{H}=3.015 \Delta x$. As shown in Fig. 32, the crack starts branching up $70.2^{\circ}$ with respect to the horizontal axis at $t=30 \mu \mathrm{s}$. As time progresses, the crack continues propagating in the same direction and the crack reaches the top edge of the plate at $t=80 \mu \mathrm{s}$.

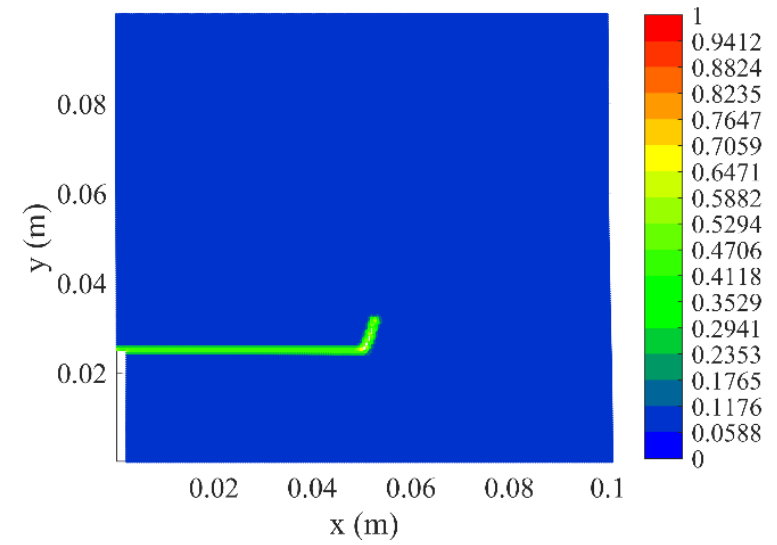

(a)

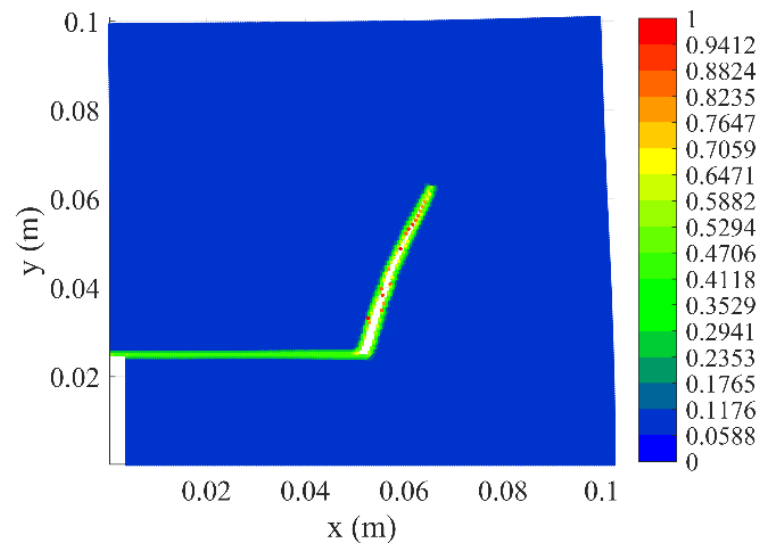

(b) 


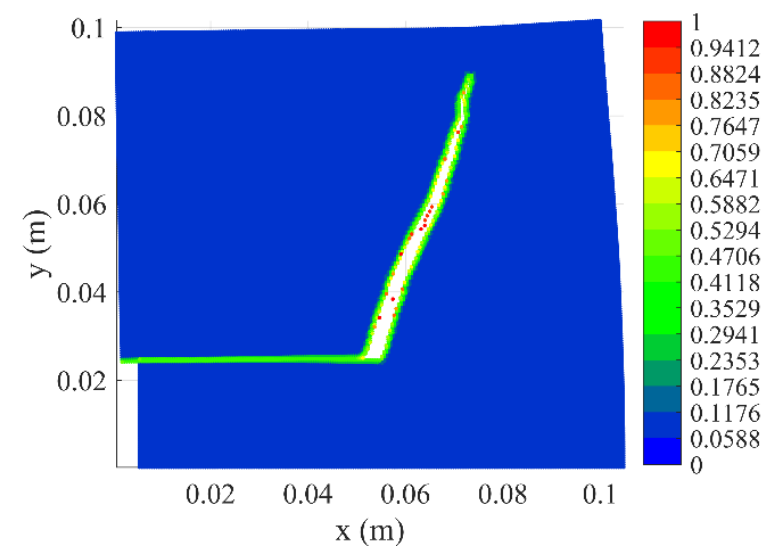

(c)

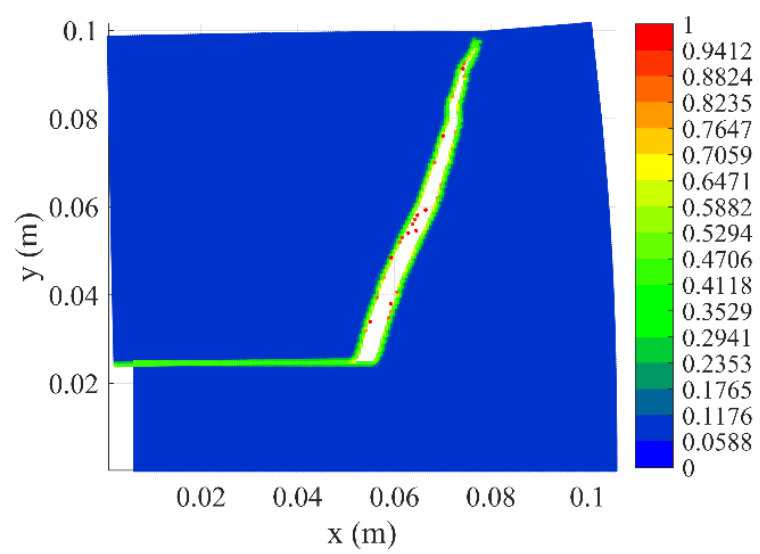

(d)

Fig. 32. Crack evolution at different times (a) $t=30 \mu \mathrm{s}$, (b) $t=50 \mu \mathrm{s}$, (c) $t=70 \mu \mathrm{s}$, (d) $t=80 \mu s$ (displacements are magnified by 5 for the deformed configuration)

Fig. 33 shows the $\delta$-convergence study in term of crack path. In this convergence study, the nodal density is kept constant $(m=\delta / \Delta x=3)$ [64-66], meanwhile the horizon size is taken as smaller and smaller $(\delta \rightarrow 0)$ [64-66]. As it can be seen from the Figs. 33(c-d), when the horizon size is small the predicted crack paths are $70.2^{\circ}$ with respect to the horizontal axis and it agrees very well with the experimental observations in [54-56], which is around $70^{\circ}$.

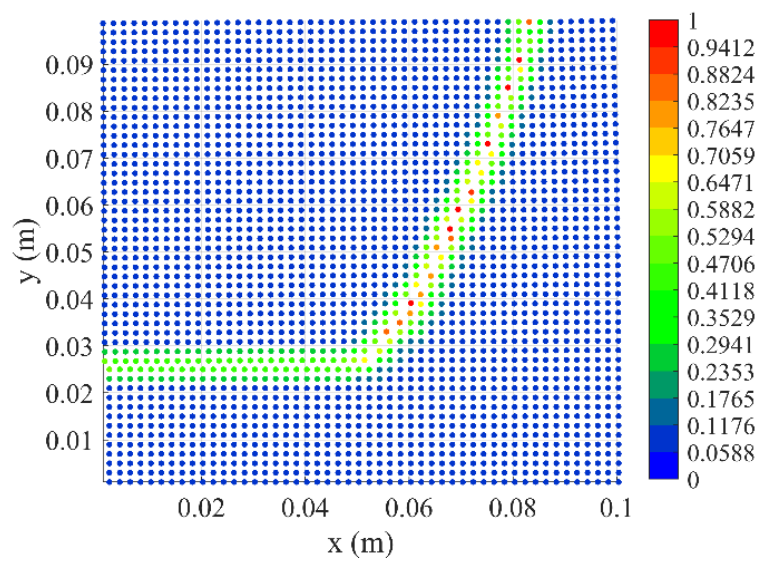

(a)

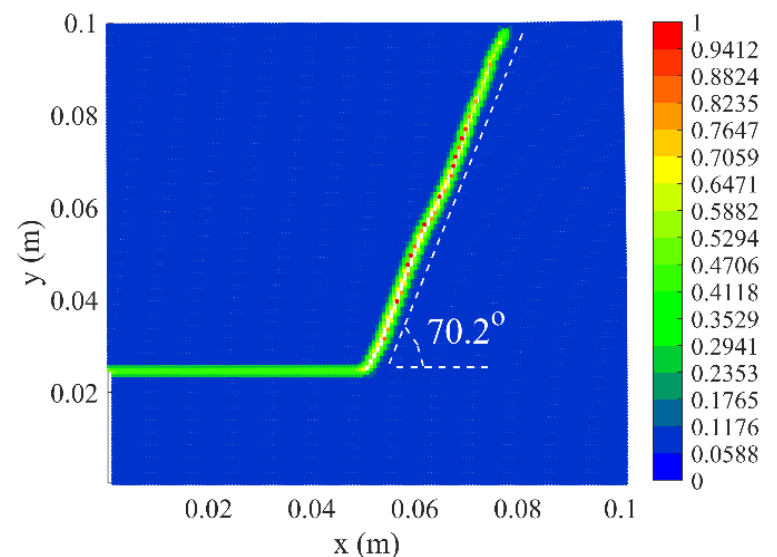

(c)

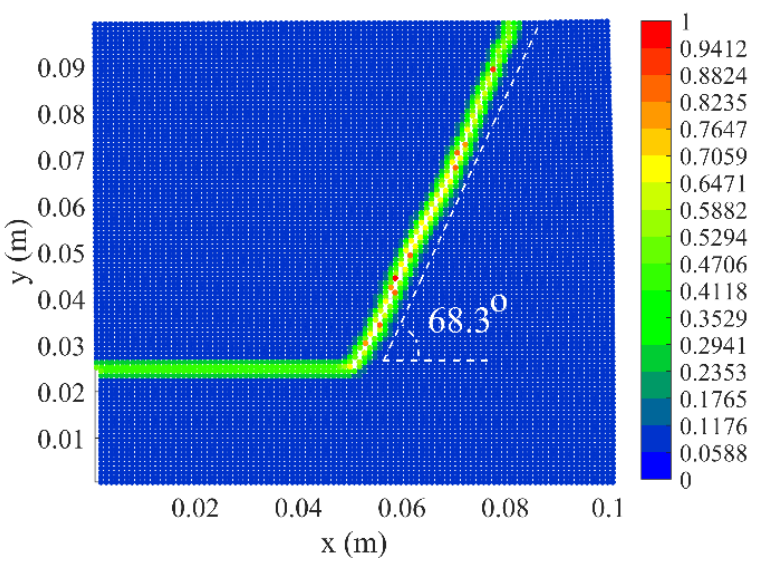

(b)

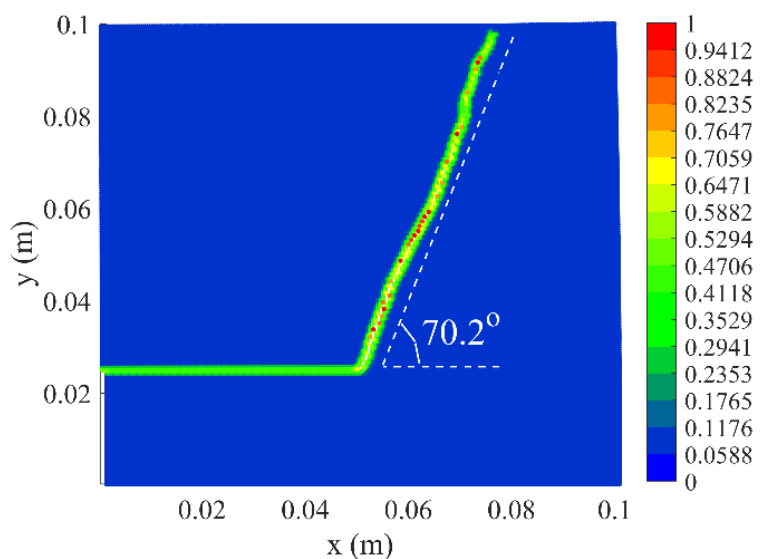

(d)

Fig. 33. $\delta$-convergence in terms of crack paths with $m=\delta / \Delta x=3$ and horizon size (a): $\delta=0.006 \mathrm{~m},(\mathrm{~b}): \delta=0.003 \mathrm{~m},(\mathrm{c}): \delta=0.002 \mathrm{~m},(\mathrm{~d}): \delta=0.0015 \mathrm{~m}$ 
6.5. Damage prediction for an L-shape plate subjected to large deformation

In this section, damage in an L-shape plate subjected to large deformation, as shown in Fig. 34, is predicted. The L-shape plate has dimensions of $L=10 \mathrm{~m}, W=2$ mand thickness of $h=0.1 \mathrm{~m}$. The material has Young's modulus of $E=1.0667 \times 10^{4} \mathrm{~N} / \mathrm{m}^{2}$, Poisson's ratio of $v=0.333$ and critical energy release rate of $G_{c}=2.7 \times 10^{3} \mathrm{~J} / \mathrm{m}^{2}$ [67]. The bottom edge of the plate is fixed and the right edge of the plate is attached to a stiff plate, shown in blue in Fig. 34

In the PD model, plane stress condition is considered. Similar to Section 6.2, in order to apply boundary conditions, three fictitious layers of material are generated at the bottom and all displacement components of the fictitious material points as well as material points located at the location at $y=0$ are set equal to zero. In order to represent the stiff plate at the right edge, Young's modulus of the stiff plate is considered as $E=1.0667 \times 10^{5} \mathrm{~N} / \mathrm{m}^{2}$.

The plate is subjected to incremental vertical displacement at $(x=L, y=L-W / 2)$ as

$v^{(n)}=v^{(n-1)}+\Delta v(\mathrm{~m})$

with

$$
\begin{aligned}
& v^{(0)}=10.075(\mathrm{~m}) \\
& \Delta v=0.001(\mathrm{~m})
\end{aligned}
$$

where $v^{(n)}$ represents applied displacement at $n^{\text {th }}$ load step $(n=1,2 \ldots 2900), \Delta v$ represents the incremental value of applied displacement, $v^{(0)}$ represents the first value of vertical displacement applied for the plate.

In the PD model, the plate is discretized with the mesh size of $\Delta x=W / 20$. The material point located at ( $x=L, y=L-W / 2$ ) is subjected to the incremental displacement given in Eq. (35). The Adaptive Dynamic Relaxation (ADR) method [60, 61] is used to simulate this quasi-static problem. In order to ensure the ADR solution is converged at each load step, the PD solution is run over 10000 time steps for each incremental displacement.

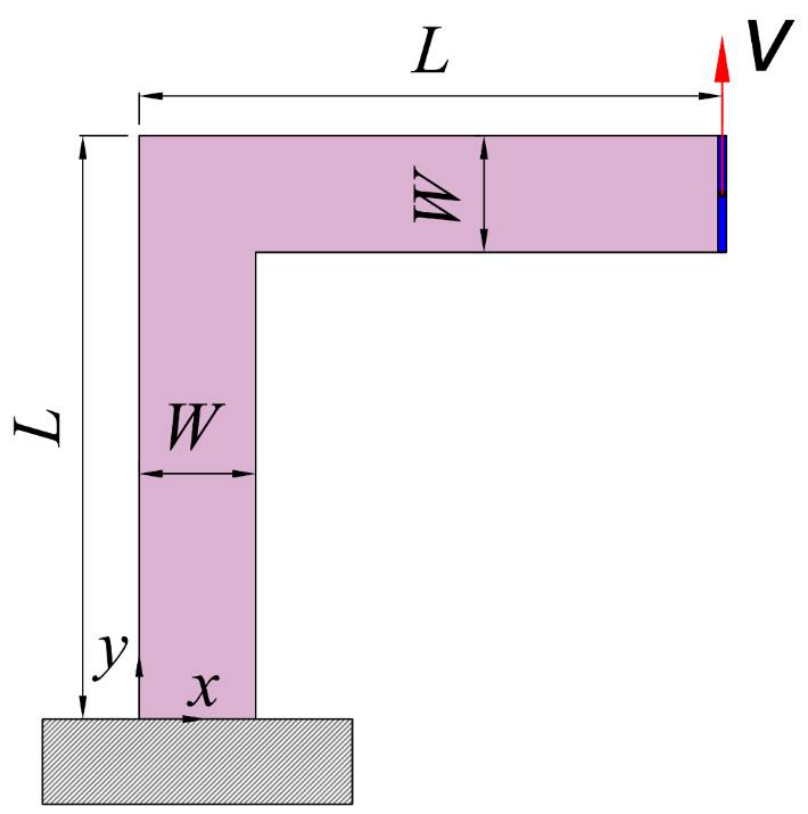

Fig. 34. L-shape plate subjected to large deformation 
Fig. 35 shows the damage evolution on the plate subjected to large deformations. As shown in Fig. 35(a), when the applied displacement is $v=10.175 \mathrm{~m}$, damage initiates around the inner corner of the plate, at $(x=W, y=L-W / 2)$. As the applied displacement is increased, the crack propagates toward the outer corner of the L-shape plate as shown in Fig. 35(b-d). When the applied displacement is $v=12.975 \mathrm{~m}$, the crack opens largely and the L-shape plate is almost split into two parts as shown in Fig. 35(d). It is observed that the damage evolution captured by the nonlinear PD model agrees very well with the previous study [67].

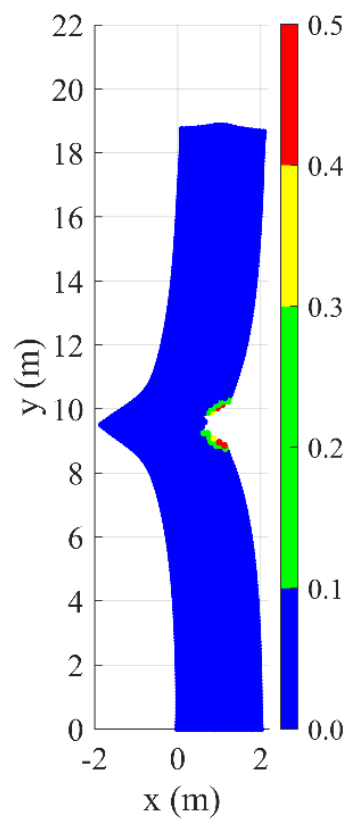

(a)

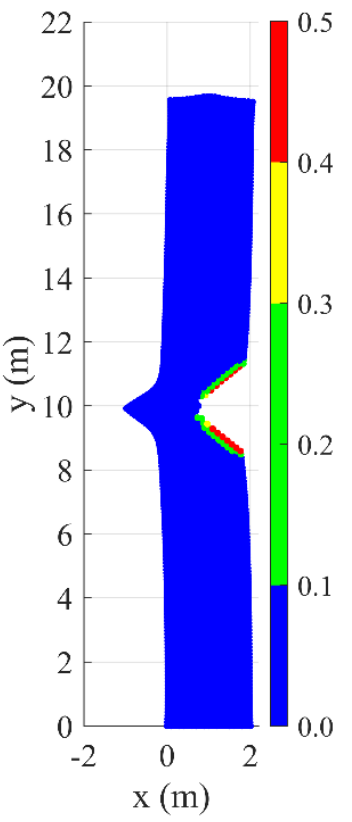

(b)

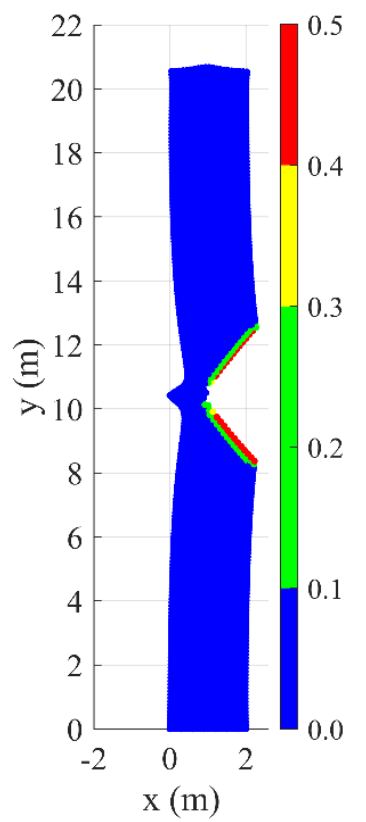

(c)

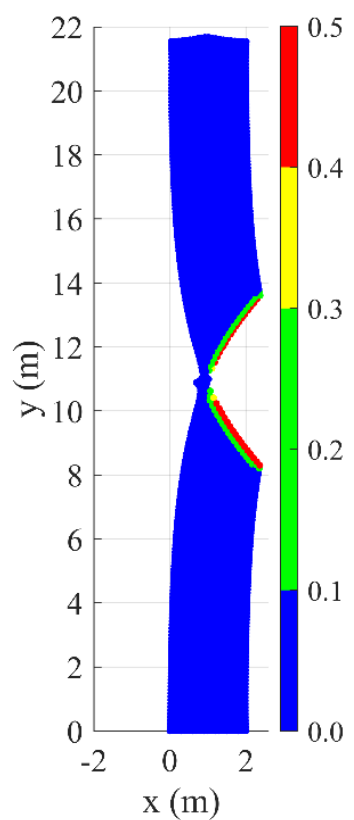

(d)

Fig. 35. Crack evolution on the L-shape plate in the deformed configuration when the applied displacement is (a) $v=10.175 \mathrm{~m}$, (b) $v=10.975 \mathrm{~m}$, (c) $v=11.975 \mathrm{~m}$, (d) $v=12.975 \mathrm{~m}$

6.6. Damage prediction in 3D pre-notched concrete beam subjected to bending

After verifying the accuracy of the developed nonlinear PD model for 3D structures, damage on a 3D structure is predicted. The experiment presented by Jenq and Shah [57] for a prenotched concrete beam is simulated by using the developed PD model. The concrete beam has dimensions of $L \times B \times H=304.8 \times 28.6 \times 70.2 \mathrm{~mm}^{3}$ and the pre-notch has the height of $a=35.1 \mathrm{~mm}$ as shown in Fig. 36. The beam is placed on two rigid cylinders located at $x=0.1 \mathrm{~L}$ and $x=0.9 L$. The material has Young's modulus $E=30 \times 10^{9} \mathrm{~N} / \mathrm{m}^{2}$, Poisson's ratio $v=0.2$ and critical energy release rate $G_{c}=20.7368 \mathrm{~J} / \mathrm{m}[57]$. 


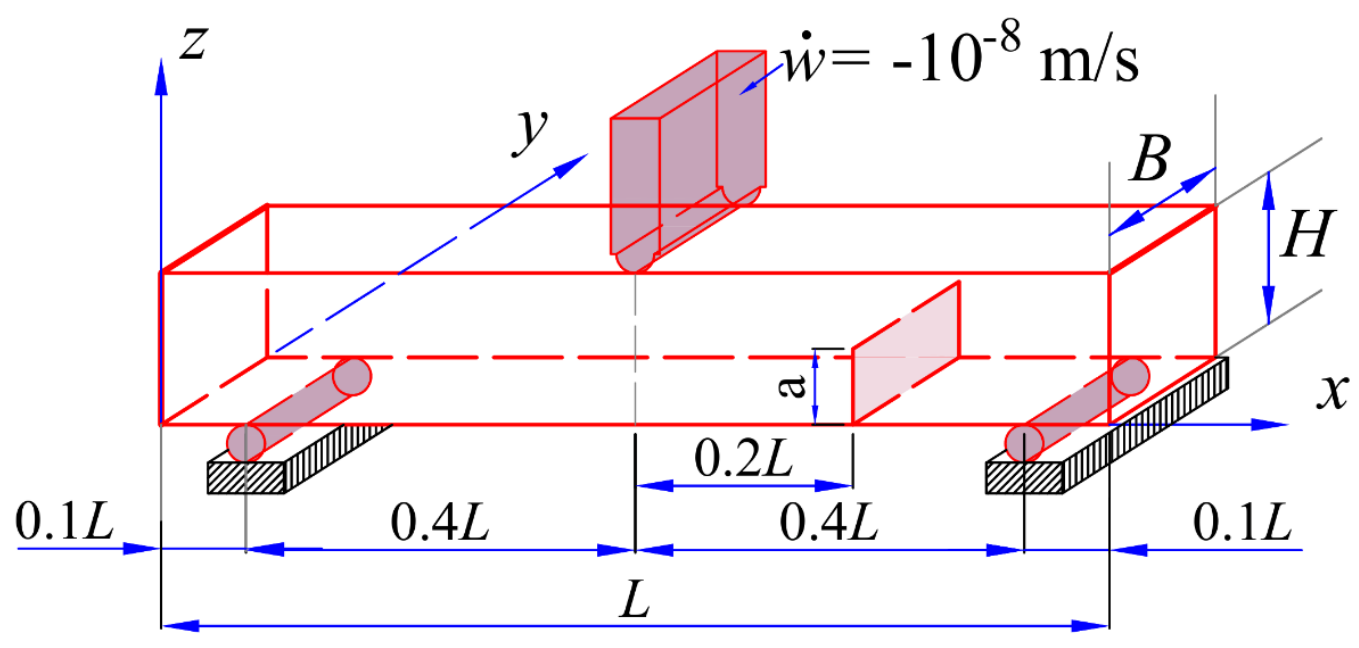

Fig. 36. 3D beam with pre-notched

In the PD model, the structure is discretized with uniform $101 \times 10 \times 24$ material points. In order to create the notch, the material points located at $x=0.7 L, 0 \leq y \leq B$ and $z \leq a$ are removed from the model.

The loading is applied by increasing the displacement by $\Delta w=-10^{-8}$ at $x=0.5 L, 0 \leq y \leq B$, $z=H$ for each load step. Zero vertical displacement, $w=0$ is applied at $x=0.1 L, 0 \leq y \leq B$ ,$z=0$ and $x=0.9 L, 0 \leq y \leq B, z=0$. The explicit time integration is used for this quasi-static problem by using the adaptive dynamic relaxation (ADR) method [60, 61].

Figs. 37-42 present the crack evolution for the concrete beam. In Figs. 37(b)-42(b), blue regions represent initial notch and red regions represent the new crack surfaces. The new crack surfaces are represented by the material points with the damage coefficient $\phi \geq 0.3$.

As shown in Fig. 37(a), when the applied displacement is $w(0.5 L, y, H)=-2 \times 10^{-6} \mathrm{~m}$, the crack propagates towards the middle section of the beam and reaches the location $(x=0.206, y, z=0.046)$. The peridynamic results show that the failure angle is $\beta=34^{\circ}$ which is similar to the failure angle observed in experiments [57].

As time progresses, the crack continues propagating in the same direction and reaches the location $(x=0.1962, y, z=0.0582)$ when $w(0.5 L, y, H)=-2.5 \times 10^{-6} \mathrm{~m}$ as shown in Fig. 38 . The crack propagates to the location of $(x=0.1928, y, z=0.0625)$ when $w(0.5 L, y, H)=-3 \times 10^{-6} \mathrm{~m}$ as shown in Fig. 39. The crack propagated to the location $(x=0.1869, y, z=0.0691)$ when $w(0.5 L, y, H)=-4 \times 10^{-6} \mathrm{~m}$ as shown in Fig. 41, then propagated towards the top surface of the beam when $w(0.5 L, y, H)=-4.5 \times 10^{-6} \mathrm{~m}$ as shown in Fig. 42. 


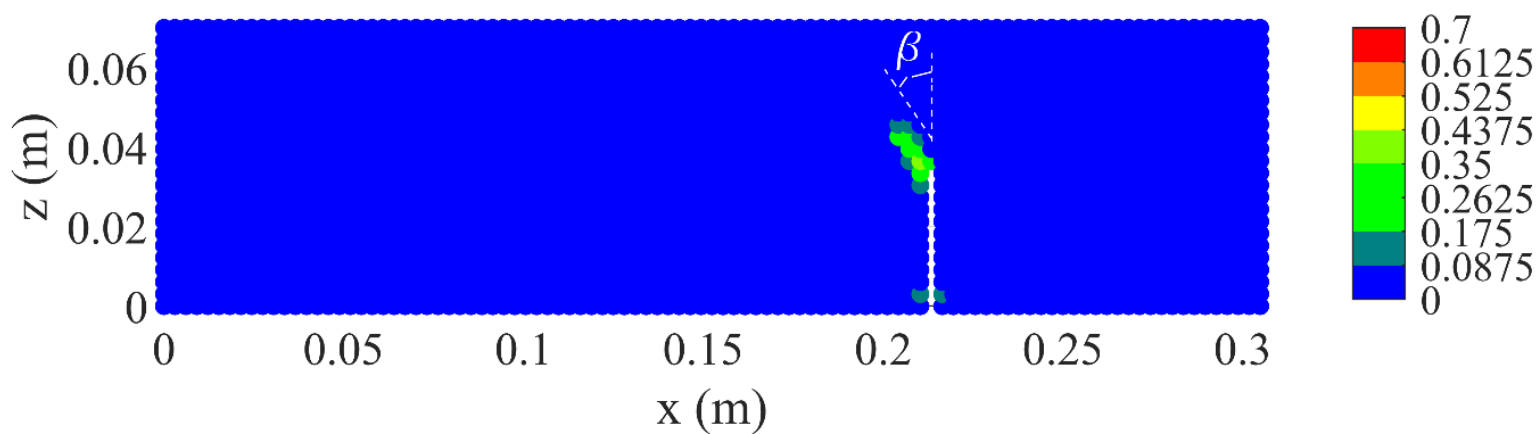

(a)

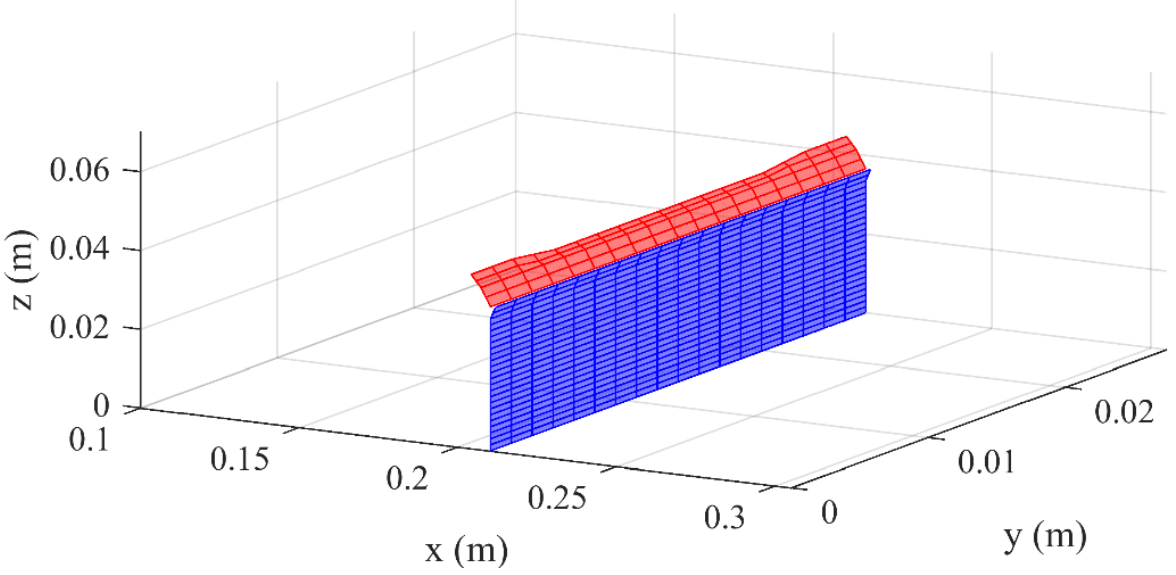

(b)

Fig. 37. Crack evolution when $w(0.5 L, y, H)=-2 \times 10^{-6} \mathrm{~m}$ (a) front view for material points located at $y=B / 2$ (b) $3 \mathrm{D}$ crack surface
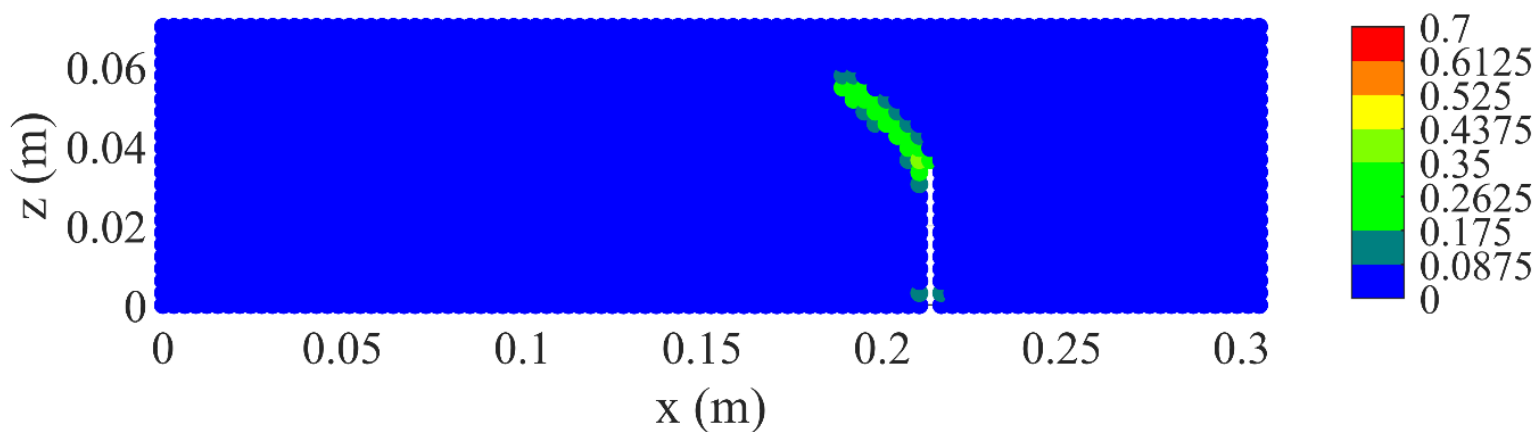

(a) 


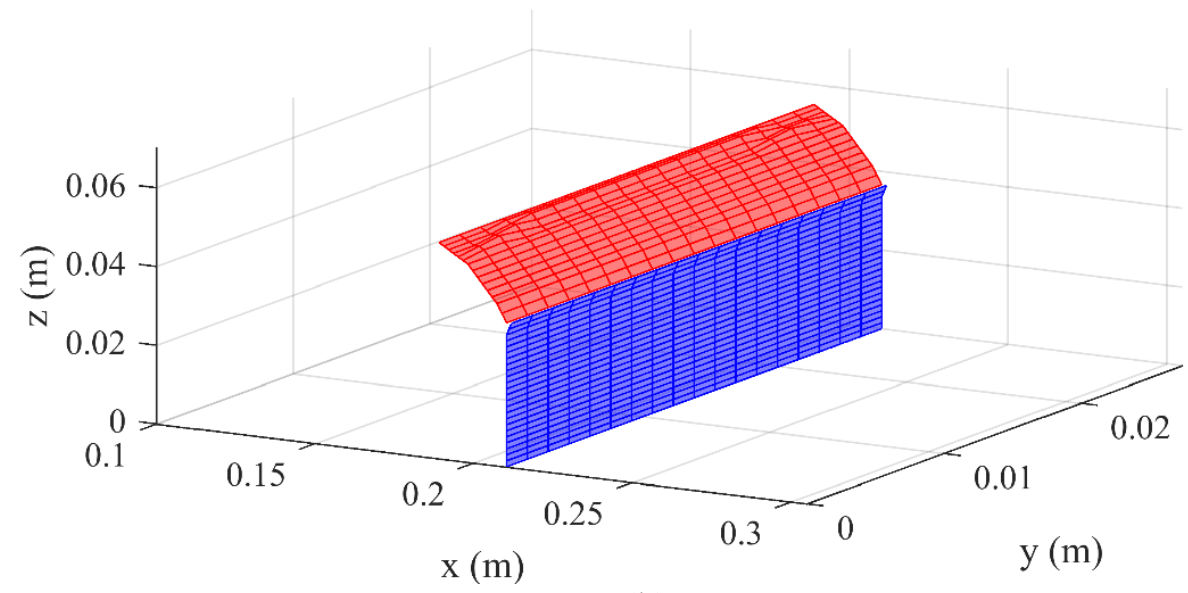

(b)

Fig. 38. Crack evolution when $w(0.5 L, y, H)=-2.5 \times 10^{-6} \mathrm{~m}$ (a) front view for material points located at $y=B / 2$ (b) $3 \mathrm{D}$ crack surface

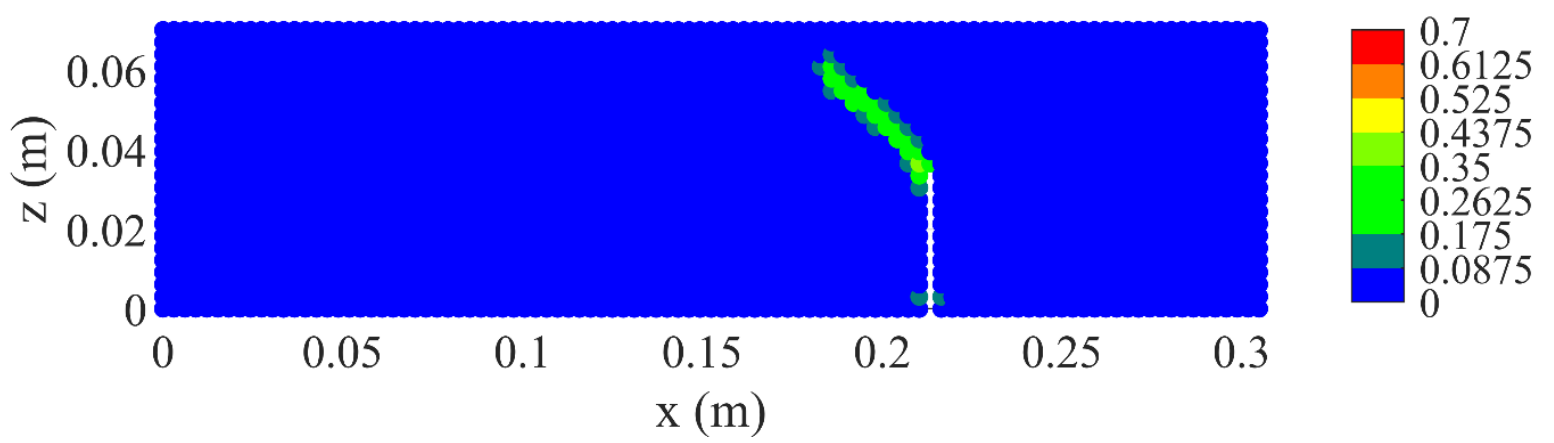

(a)

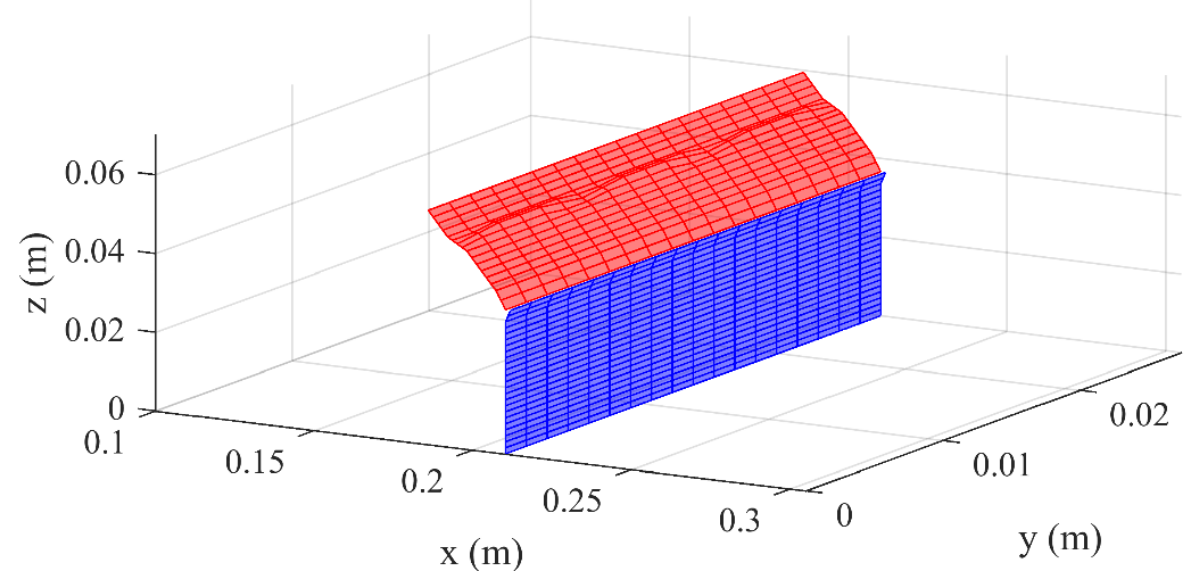

(b)

Fig. 39. Crack evolution when $w(0.5 L, y, H)=-3 \times 10^{-6} \mathrm{~m}$ (a) front view for material points located at $y=B / 2$ (b) $3 \mathrm{D}$ crack surface 


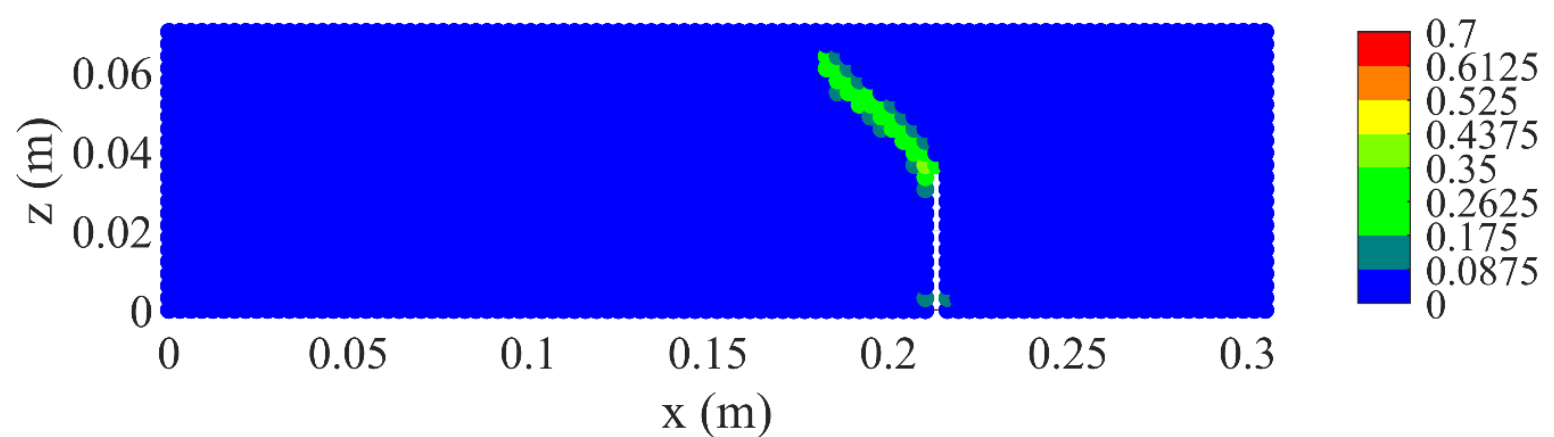

(a)

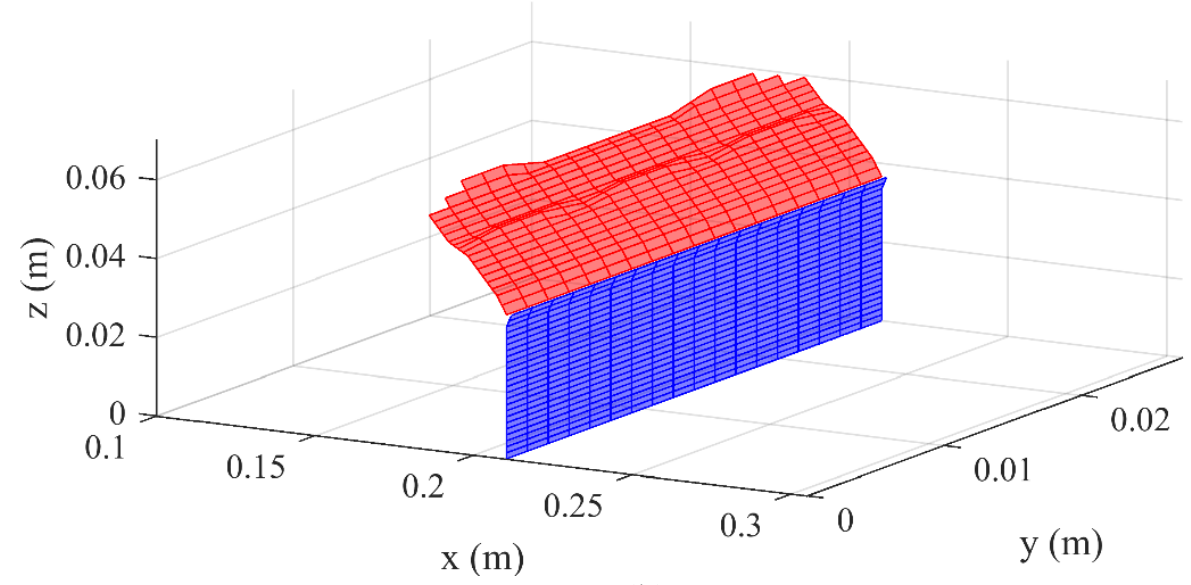

(b)

Fig. 40. Crack evolution when $w(0.5 L, y, H)=-3.5 \times 10^{-6} \mathrm{~m}$ (a) front view for material points located at $y=B / 2$ (b) $3 \mathrm{D}$ crack surface

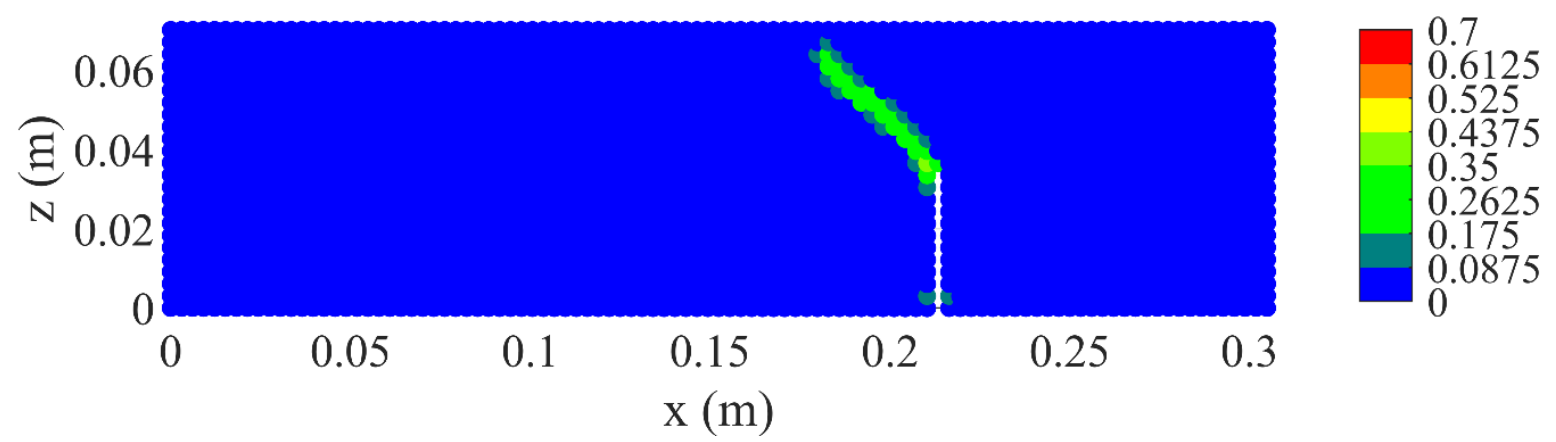

(a) 


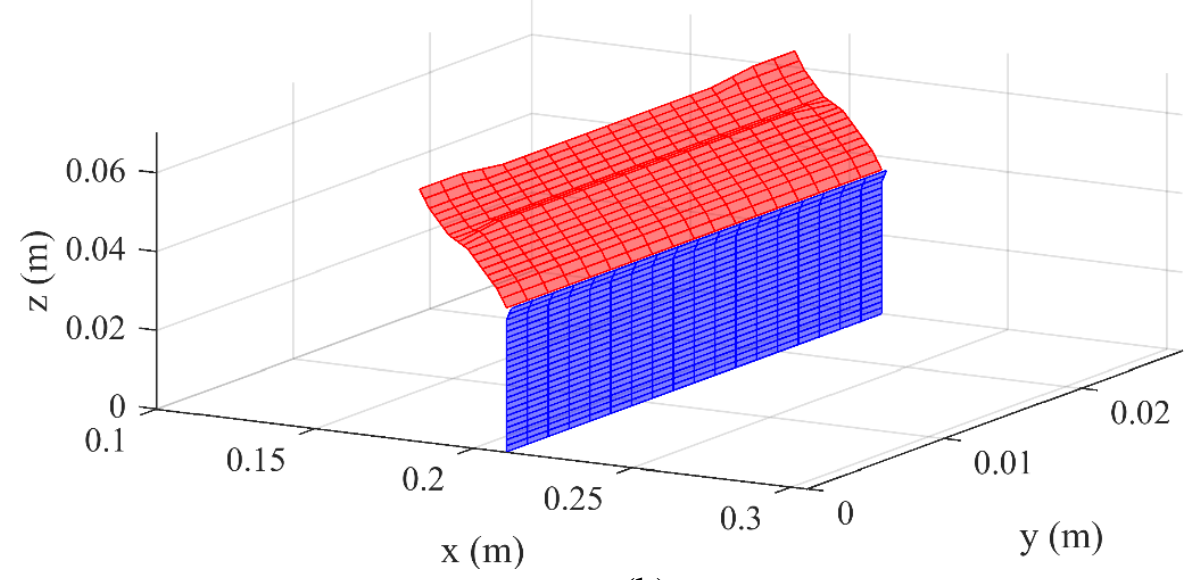

(b)

Fig. 41. Crack evolution when $w(0.5 L, y, H)=-4 \times 10^{-6} \mathrm{~m}$ (a) front view for material points located at $y=B / 2$ (b) $3 \mathrm{D}$ crack surface
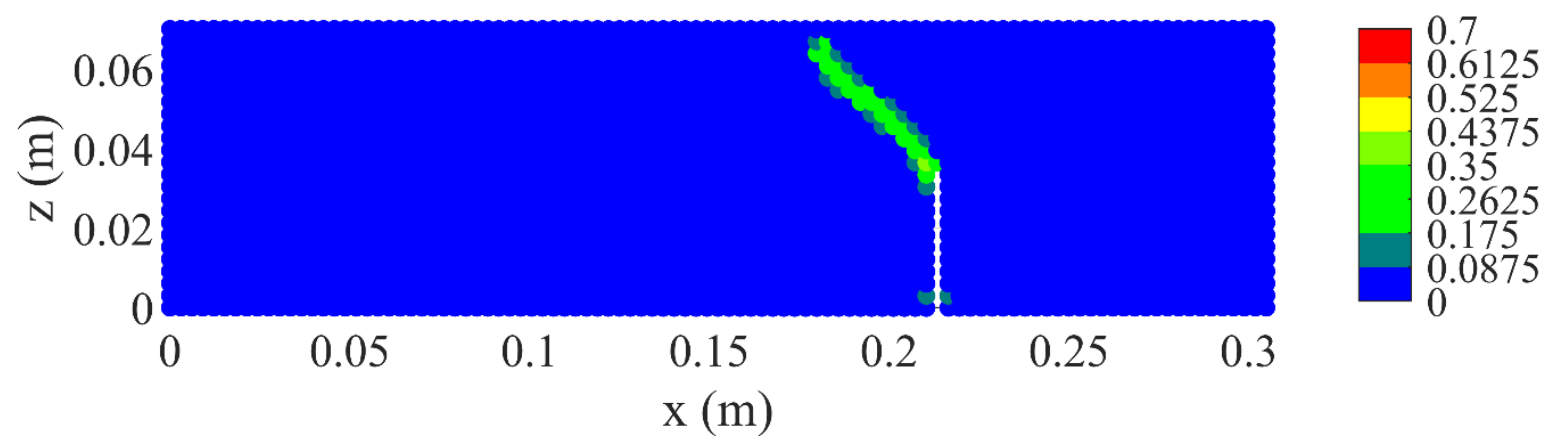

(a)

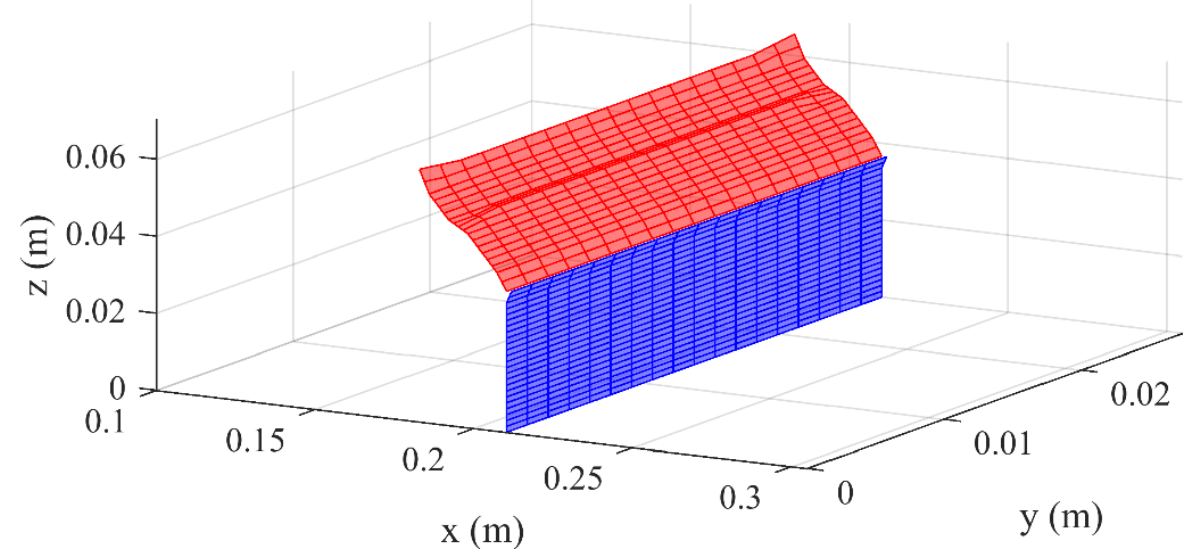

(b)

Fig. 42. Crack evolution when $w(0.5 L, y, H)=-4.5 \times 10^{-6} \mathrm{~m}$ (a) front view for material points located at $y=B / 2$ (b) $3 \mathrm{D}$ crack surface

\section{Concluding remarks}

This study presents a novel ordinary state-based peridynamic model for geometrically nonlinear analysis. A logarithmic bond stretch has been proposed for large deformation 
problems. The peridynamic formulations for 1D, 2D, and 3D structures are obtained based on principle of virtual displacements by using Total Lagrange formulation. The accuracy of the developed nonlinear PD model is verified by comparing it to nonlinear FEA solutions.

For damage prediction, first, the developed nonlinear PD model is used to predict damages for a plate subjected to dynamic loading and an L-shape plate subjected to large deformation. Then, damage pattern in 3D pre-notched concrete beam subjected to quasi-static loading condition is predicted. All results show very good agreement with experimental results.

\section{Acknowledgment}

The authors gratefully acknowledge financial support from the Ministry of Education and Training in Vietnam and the University of Strathclyde. 


\section{Appendix A. Principle of virtual displacements}

As described in Section 3, the PD constants are determined by comparing the virtual values of strain energy density in PD and classical continuum mechanics based on the principle of virtual displacements. Therefore, this section presents the formulation of the virtual values of strain energy density in classical continuum mechanics based on the principle of virtual displacements.

According to Bathe [1], the equation of motion in classical continuum mechanics can be derived by using the principle of virtual displacements as

$\int^{t+\Delta t} V\left({ }^{t+\Delta t} \sigma_{i j}\right) \delta\left({ }^{t+\Delta t} e_{i j}\right) d\left({ }^{t+\Delta t} V\right)={ }^{t+\Delta t} \mathfrak{R}$

where ${ }^{t+\Delta t} \sigma_{i j}$ represents Cartesian components of Cauchy stress tensor, $\delta^{t+\Delta t} e_{i j}$ represents components of strain tensor corresponding to virtual displacements, ${ }^{t+\Delta t} V$ represents volume at time $t+\Delta t$. The parameter ${ }^{t+\Delta t} \mathfrak{R}$ represents the external virtual work which can be calculated as [1]

${ }^{t+\Delta t} \mathfrak{R}=\int_{{ }^{t+\Delta t} V}\left({ }^{t+\Delta t} \mathbf{f}^{B}\right)(\delta \mathbf{u}) d\left({ }^{t+\Delta t} V\right)+\int_{{ }^{t+\Delta t} S}\left({ }^{t+\Delta t} \mathbf{f}^{S}\right)\left(\delta \mathbf{u}^{s}\right) d\left({ }^{t+\Delta t} S\right)$

where ${ }^{t+\Delta t} \mathbf{f}^{B}$ and ${ }^{t+\Delta t} \mathbf{f}^{S}$ represent external force per unit volume and external force per unit surface area at time $t+\Delta t$, respectively. ${ }^{t+\Delta t} S$ represents surface at time $t+\Delta t$ on which the external force per unit surface area is applied, $\delta \mathbf{u}^{s}$ represents virtual displacement evaluated on the surface ${ }^{t+\Delta t} S$.

Since the configuration at time $t+\Delta t$ is unknown, the principle of virtual displacements given in Eq. (A1) needs to be rewritten with respect to a known configuration. According to Bathe [1], if the configuration at time $\tau_{0}$ is known, the following relation is obtained

$\int_{{ }^{t+\Delta V} V}\left({ }^{t+\Delta t} \sigma_{i j}\right) \delta\left({ }^{t+\Delta t} e_{i j}\right) d\left({ }^{t+\Delta t} V\right)=\int_{\tau_{0} V}\left({ }^{t+\Delta t}{ }_{\tau_{0}} S_{i j}\right) \delta\left({ }^{t+\Delta t}{ }_{\tau_{0}} \varepsilon_{i j}\right) d\left({ }^{\tau_{0}} V\right)$

where ${ }^{t+\Delta t} S_{i j}$ and ${ }_{\tau_{0}}^{t+\Delta t} \varepsilon_{i j}$ represent components of Second Piola-Kirchhoff (SPK) stress and Green Lagrange (GL) strain with respect to the configuration at time $\tau_{0}, \delta\left({ }_{\tau_{0}}^{t+\Delta t} \varepsilon_{i j}\right)$ represents the virtual value of GL strain.

If Eq. (A3) is written with respect to the initial configuration $\left(\tau_{0}=0\right)$, it is referred as Total Lagrangian (TL) formulation [1]

$\int_{{ }^{0} V}\left({ }^{t+\Delta t} S_{i j}\right) \delta\left({ }^{t+\Delta t} \varepsilon_{i j}\right) d\left({ }^{0} V\right)={ }^{t+\Delta t} \mathfrak{R}$

If Eq. (A3) is written with respect to the previous configuration $\left(\tau_{0}=t\right)$, it is referred as Updated Lagrangian (UL) formulation [1]

$\int_{{ }^{t} V}\left({ }^{t+\Delta t} S_{i j}\right) \delta\left({ }^{t+\Delta t}{ }_{t} \varepsilon_{i j}\right) d\left({ }^{t} V\right)={ }^{t+\Delta t} \mathfrak{R}$

In Eq. (A4) for TL formulation, the stress components can be decomposed as ${ }_{0}^{t+\Delta t} S_{i j}={ }_{0}^{t} S_{i j}+{ }^{t+\Delta t} S_{i j}$

where ${ }_{0}^{t} S_{i j}$ represents SPK stress at time $t,{ }^{t+\Delta t} S_{i j}$ represents the incremental value of SPK stress from time $t$ to time $t+\Delta t$.

Similarly, the strain components can be decomposed as

${ }_{0}^{t+\Delta t} \varepsilon_{i j}={ }_{0}^{t} \varepsilon_{i j}+{ }^{t+\Delta t}{ }_{t} \varepsilon_{i j}={ }_{0}^{t} \varepsilon_{i j}+{ }^{t+\Delta t} e_{i j}+{ }^{t+\Delta t}{ }_{t} \eta_{i j}$ 
where ${ }_{0}^{t} \varepsilon_{i j}$ represents Green Lagrange at time $t,{ }^{t+\Delta t} e_{i j}$ and ${ }^{t+\Delta t} \eta_{i j}$ represent the incremental linear and nonlinear strains from time $t$ to time $t+\Delta t$. These incremental strains can be represented as [1]

$$
\begin{aligned}
& { }_{t}^{t+\Delta t} e_{i j}=\frac{1}{2}\left[\left({ }^{t+\Delta t}{ }_{t} u_{i, j}\right)+\left({ }^{t+\Delta t} u_{j, i}\right)+\left({ }_{0}^{t} u_{k, i}\right)\left({ }^{t+\Delta t}{ }_{t} u_{k, j}\right)+\left({ }_{0}^{t} u_{k, j}\right)\left({ }^{t+\Delta t} u_{k, i}\right)\right] \\
& { }^{t+\Delta t} \eta_{i j}=\frac{1}{2}\left({ }^{t+\Delta t}{ }_{t} u_{k, i}\right)\left({ }^{t+\Delta t}{ }_{t} u_{k, j}\right)
\end{aligned}
$$

with

$$
{ }^{t+\Delta t}{ }_{t} u_{i, j}=\frac{\partial\left({ }^{t+\Delta t}{ }_{t} u_{i}\right)}{\partial\left({ }^{0} x_{j}\right)}
$$

Note that the virtual value of the Green Lagrange strain given in Eq. (A4) can be written as [1] $\delta\left({ }_{0}^{t+\Delta t} \varepsilon_{i j}\right)=\delta\left({ }^{t+\Delta t} \varepsilon_{i j}\right)$

Therefore, by using relations given in Eqs. (A6-A9), the equation of motion in TL formulation given in Eq. (A4) can be rewritten as [1]

$\int_{{ }^{0} V}\left({ }^{t+\Delta t}{ }_{t} S_{i j}\right) \delta\left({ }^{t+\Delta t}{ }_{t} \varepsilon_{i j}\right) d\left({ }^{0} V\right)+\int_{{ }^{0} V}\left({ }_{0}^{t} S_{i j}\right) \delta\left({ }^{t+\Delta t}{ }_{t} \eta_{i j}\right) d\left({ }^{0} V\right)={ }^{t+\Delta t} \mathfrak{R}-\int_{{ }^{0} V}\left({ }_{0}^{t} S_{i j}\right) \delta\left({ }^{t+\Delta t}{ }_{t} e_{i j}\right) d\left({ }^{0} V\right)$

(A10)

By using Taylor series expansion and ignoring the higher-order terms, the following approximation for SPK stress is obtained [1]

$$
\begin{aligned}
{ }_{t}^{t+\Delta t} S_{i j} & \approx \frac{\partial\left({ }_{0}^{t} S_{i j}\right)}{\partial\left({ }_{0}^{t} \varepsilon_{r s}\right)}\left({ }^{t+\Delta t}{ }_{t} \varepsilon_{r s}\right) \approx \frac{\partial\left({ }_{0}^{t} S_{i j}\right)}{\partial\left({ }_{0}^{t} \varepsilon_{r s}\right)}\left({ }^{t+\Delta t} e_{r s}+{ }^{t+\Delta t} \eta_{r s}\right) \\
& \approx \frac{\partial\left({ }_{0}^{t} S_{i j}\right)}{\partial\left({ }_{0}^{t} \varepsilon_{r s}\right)}\left({ }^{t+\Delta t} e_{r s}\right) \approx\left({ }_{0}^{t} C_{i j r s}\right)\left({ }^{t+\Delta t} e_{r s}\right)
\end{aligned}
$$

where ${ }_{0}^{t} C_{i j r s}$ represents the incremental stress-strain tensor at time $t$ with respect to the initial configuration.

Similarly, the virtual value of Green Lagrange strain can be approximated as [1]

$$
\delta\left({ }^{t+\Delta t}{ }_{t} \varepsilon_{i j}\right)=\delta\left({ }^{t+\Delta t} e_{i j}+{ }^{t+\Delta t} \eta_{i j}\right) \approx \delta\left({ }^{t+\Delta t} e_{i j}\right)
$$

Therefore, by using relations given in Eqs. (A11-12), the equation of motion given in Eq. (A10) can be written as [1]

$$
\begin{aligned}
{ }^{t+\Delta t} \mathfrak{R}-\int_{0_{V}}\left({ }_{0}^{t} S_{i j}\right) \delta\left({ }^{t+\Delta t} e_{i j}\right) d\left({ }^{0} V\right)= & \int_{{ }^{{ }^{V}}}\left({ }_{0}^{t} C_{i j r s}\right)\left({ }^{t+\Delta t} e_{r s}\right) \delta\left({ }^{t+\Delta t} e_{i j}\right) d\left({ }^{0} V\right) \\
& +\int_{{ }^{0} V}\left({ }_{0}^{t} S_{i j}\right) \delta\left({ }^{t+\Delta t}{ }_{t} \eta_{i j}\right) d\left({ }^{0} V\right)
\end{aligned}
$$

According to Bathe [1], Eq. (A13) can be rewritten as

$\left(\delta \hat{\mathbf{U}}^{T}\right)\left({ }_{0}^{t} \mathbf{K}_{L}\right) \hat{\mathbf{U}}+\left(\delta \hat{\mathbf{U}}^{T}\right)\left({ }_{0}^{t} \mathbf{K}_{N L}\right) \hat{\mathbf{U}}=\left(\delta \hat{\mathbf{U}}^{T}\right)\left({ }^{t+\Delta t} \mathbf{F}_{e x t}\right)-\left(\delta \hat{\mathbf{U}}^{T}\right)\left({ }_{0}^{t} \mathbf{F}\right)$

or

$\left({ }_{0}^{t} \mathbf{K}_{L}+{ }_{0}^{t} \mathbf{K}_{N L}\right) \hat{\mathbf{U}}={ }^{t+\Delta t} \mathbf{F}_{\text {ext }}-{ }_{0}^{t} \mathbf{F}$

with

$$
\begin{aligned}
& \left(\delta \hat{\mathbf{U}}^{T}\right)\left({ }_{0}^{t} \mathbf{K}_{L}\right) \hat{\mathbf{U}}=\int_{{ }^{0} V}\left({ }_{0}^{t} C_{i j r s}\right)\left({ }^{t+\Delta t}{ }_{t} e_{r s}\right) \delta\left({ }^{t+\Delta t} e_{i j}\right) d\left({ }^{0} V\right) \\
& \left(\delta \hat{\mathbf{U}}^{T}\right)\left({ }_{0}^{t} \mathbf{K}_{N L}\right) \hat{\mathbf{U}}=\int_{{ }^{0} V}\left({ }_{0}^{t} S_{i j}\right) \delta\left({ }^{t+\Delta t} \eta_{i j}\right) d\left({ }^{0} V\right)
\end{aligned}
$$




$$
\begin{aligned}
& \left(\delta \hat{\mathbf{U}}^{T}\right){ }_{0}^{t} \mathbf{F}=\int_{{ }^{{ }^{V}}}\left({ }_{0}{ }_{0} S_{i j}\right) \delta\left({ }^{t+\Delta t} e_{i j}\right) d\left({ }^{0} V\right) \\
& \left(\delta \hat{\mathbf{U}}^{T}\right)\left({ }^{t+\Delta t} \mathbf{F}_{\text {ext }}\right)={ }^{t+\Delta t} \mathfrak{R}
\end{aligned}
$$

where $\hat{\mathbf{U}}$ represents the vector of increments for displacements, $\delta \hat{\mathbf{U}}$ represents vector of virtual displacements, ${ }_{0}^{t} \mathbf{F}$ represents the vector of point forces equivalent to the element stresses at time $t,{ }^{t+\Delta t} \mathbf{F}_{e x t}$ represents vector of externally applied point loads at time $t+\Delta t$, ${ }_{0}^{t} \mathbf{K}_{L}$ and ${ }_{0}^{t} \mathbf{K}_{N L}$ represent linear and nonlinear stiffness matrices, respectively.

For dynamic problems, the inertial force is added into Eq. (A14b) as

$$
\mathbf{M}^{t+\Delta t} \hat{\mathbf{U}}+\left({ }_{0}^{t} \mathbf{K}_{L}+{ }_{0}^{t} \mathbf{K}_{N L}\right) \hat{\mathbf{U}}={ }^{t+\Delta t} \mathbf{F}_{e x t}-{ }_{0}^{t} \mathbf{F}
$$

where $\mathbf{M}$ represents time-independent mass matrix, ${ }^{t+\Delta t} \hat{\ddot{\mathbf{U}}}$ represents the vector of acceleration at time $t+\Delta t$.

For dynamic solutions using an explicit time integration scheme, the equation of motion given in Eq. (A15) can be rewritten as [1]

$$
\mathbf{M}^{t} \hat{\ddot{\mathbf{U}}}={ }^{t} \mathbf{F}_{\text {ext }}-{ }_{0}^{t} \mathbf{F}
$$

where ${ }^{t} \mathbf{F}_{\text {ext }}$ represents the vector of externally applied point loads at time $t$ in explicit time integration, ${ }^{t} \hat{\mathbf{U}}$ represents the vector of point accelerations at time $t$.

In this study, the explicit time integration scheme as given in Eq. (A16) is used. Therefore, the virtual value of strain energy density corresponding to virtual displacements in classical continuum mechanics can be calculated by using the relation given in Eq. (A14e) as

$$
\delta W^{C C M}=\sum_{i=1}^{3} \sum_{i=1}^{3}\left({ }_{0}^{t} S_{i j}\right) \delta\left({ }^{t+\Delta t} e_{i j}\right)
$$

or

$$
\begin{aligned}
\delta W^{C C M}= & \left({ }_{0}^{t} S_{x x}\right) \delta\left({ }^{t+\Delta t} e_{x x}\right)+\left({ }_{0}^{t} S_{y y}\right) \delta\left({ }^{t+\Delta t}{ }_{t} e_{y y}\right)+\left({ }_{0}^{t} S_{z z}\right) \delta\left({ }^{t+\Delta t} e_{z z}\right) \\
& +2\left({ }_{0}^{t} S_{x y}\right) \delta\left({ }^{t+\Delta t}{ }_{t} e_{x y}\right)+2\left({ }_{0}^{t} S_{y z}\right) \delta\left({ }^{t+\Delta t}{ }_{t} e_{y z}\right)+2\left({ }_{0}^{t} S_{x z}\right) \delta\left({ }^{t+\Delta t}{ }_{t} e_{x z}\right)
\end{aligned}
$$

where the linear strain components, ${ }^{t+\Delta t} e_{i j}$ are given in Eq. (A8a). These strain components in Total Lagrangian formulation can be calculated as [1]

$$
\begin{aligned}
& { }^{t+\Delta t} e_{x x}={ }^{t+\Delta t}{ }_{t} u_{, x}+{ }_{0}^{t} u_{, x}{ }^{t+\Delta t}{ }_{t} u_{, x}+{ }_{0}^{t} v_{, x}{ }^{t+\Delta t}{ }_{t} v_{, x}+{ }_{0}^{t} w_{, x}{ }^{t+\Delta t}{ }_{t} w_{, x} \\
& { }_{t+\Delta t}^{t+} e_{y y}={ }^{t+\Delta t} v_{, y}+{ }_{0}^{t} v_{, y}{ }^{t+\Delta t}{ }_{t} v_{, y}+{ }_{0}^{t} u_{, y}{ }^{t+\Delta t}{ }_{t} u_{, y}+{ }_{0}^{t} w_{, y}{ }^{t+\Delta t}{ }_{t} w_{, y} \\
& { }_{t}^{t+\Delta t} e_{z z}={ }^{t+\Delta t}{ }_{t} w_{, z}+{ }_{0}^{t} u_{, z}{ }^{t+\Delta t}{ }_{t} u_{, z}+{ }_{0}^{t} v_{, z}{ }^{t+\Delta t}{ }_{t} v_{, z}+{ }_{0}^{t} w_{, z}{ }^{t+\Delta t}{ }_{t} w_{, z} \\
& { }^{t+\Delta t} e_{x y}=\frac{1}{2}\left({ }^{t+\Delta t}{ }_{t} u_{, y}+{ }^{t+\Delta t}{ }_{t} v_{, x}\right)+\frac{1}{2}\left(\begin{array}{l}
{ }_{0}^{t} u_{, x}{ }^{t+\Delta t}{ }_{t} u_{, y}+{ }_{0}^{t} u_{, y}{ }^{t+\Delta t}{ }_{t} u_{, x}+{ }_{0}^{t} v_{, x}{ }^{t+\Delta t}{ }_{t} v_{, y} \\
+{ }_{0}^{t} v_{, y}{ }^{t+\Delta t}{ }_{t} v_{, x}+{ }_{0}^{t} w_{, x}{ }^{t+\Delta t}{ }_{t} w_{, y}+{ }_{0}{ }^{t} w_{, y}{ }^{t+\Delta t}{ }_{t} w_{, x}
\end{array}\right) \\
& { }^{t+\Delta t} e_{y z}=\frac{1}{2}\left({ }_{t}^{t+\Delta t} v_{, z}+{ }_{t}^{t+\Delta t}{ }_{t} w_{, y}\right)+\frac{1}{2}\left(\begin{array}{c}
{ }_{0}^{t} u_{, z}{ }^{t+\Delta t}{ }_{t} u_{, y}+{ }_{0}^{t} u_{, y}{ }^{t+\Delta t}{ }_{t} u_{, z}+{ }_{0}^{t} v_{, z}{ }^{t+\Delta t}{ }_{t} v_{, y} \\
+{ }_{0}^{t} v_{, y}{ }^{t+\Delta t}{ }_{t} v_{, z}+{ }_{0}{ }^{t} w_{, y}{ }^{t+\Delta t}{ }_{t} w_{, z}+{ }_{0}^{t} w_{, z}{ }^{t+\Delta t}{ }_{t} w_{, y}
\end{array}\right)
\end{aligned}
$$




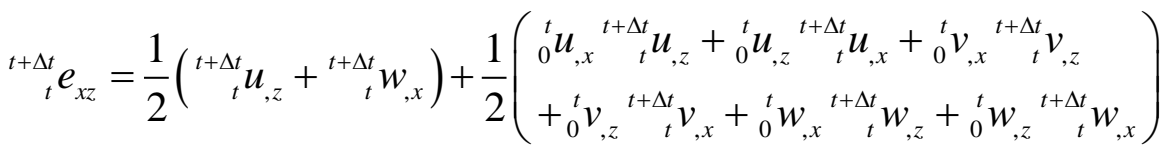

The SPK stress, ${ }_{0}^{t} S_{i j}$ in Eq. (A17) can be calculated by using the following procedure [1]:

Step 1: Calculate deformation gradient, ${ }_{0}^{t} \mathbf{X}$ by using known displacement field at time $t$

Step 2: Using the relation ${ }_{0}^{t} \mathbf{X}={ }_{0}^{t} \mathbf{R}{ }_{0}^{t} \mathbf{U}$, find rotational matrix, ${ }_{0}^{t} \mathbf{R}$ and right stretch tensor, ${ }_{0}^{t} \mathbf{U}$ by using polar decomposition [1]

2.1. Find right Cauchy-Green deformation tensor, ${ }_{0}^{t} \mathbf{C}$

$$
{ }_{0}^{t} \mathbf{C}={ }_{0}^{t} \mathbf{X}^{T}{ }_{0}^{t} \mathbf{X}
$$

2.2. Find eigenvalues, ${ }_{0}^{t} \boldsymbol{\lambda}$ and eigenvectors, $\mathbf{P}$ of ${ }_{0}^{t} \mathbf{C}$ by solving the following equation ${ }_{0}^{t} \mathbf{C P}=\mathbf{P}_{0}^{t} \boldsymbol{\lambda}$

2.3. Find rotational matrix, ${ }_{0}^{t} \mathbf{R}$, and right stretch tensor, ${ }_{0}^{t} \mathbf{U}$

- Find the deformation gradient in the principal coordinates, ${ }_{0}^{t} \mathbf{X}^{\prime}$

${ }_{0}^{t} \mathbf{X}^{\prime}=\mathbf{P}^{T}{ }_{0}^{t} \mathbf{X} \mathbf{P}$

- Find rotational matrix and right stretch tensor

$$
\begin{aligned}
& { }_{0}^{t} \mathbf{R}=\mathbf{P} \mathbf{R}^{\prime} \mathbf{P}^{T} \\
& { }_{0}^{t} \mathbf{U}=\mathbf{P} \boldsymbol{\Lambda} \mathbf{P}^{T}
\end{aligned}
$$

with

$$
\begin{aligned}
& \boldsymbol{\Lambda}=\left({ }_{0}^{t} \boldsymbol{\lambda}\right)^{1 / 2} \\
& \mathbf{R}^{\prime}={ }_{0}^{t} \mathbf{X}^{\prime}\left({ }_{0}^{t} \boldsymbol{\lambda}\right)^{-1 / 2}
\end{aligned}
$$

Step 3: Find Hencky strain tensor, ${ }_{0}^{t} \mathbf{E}^{H}$ [1]

$$
{ }_{0}^{t} \mathbf{E}^{H}=\mathbf{P}(\ln (\boldsymbol{\Lambda})) \mathbf{P}^{T}
$$

Mean strain:

${ }_{0}^{t} E_{m}^{H}=\frac{1}{3} \operatorname{tr}\left({ }_{0}^{t} \mathbf{E}^{H}\right)$

Deviatoric strain:

$$
{ }_{0}^{t} \mathbf{E}_{d}^{H}={ }_{0}^{t} \mathbf{E}^{H}-{ }_{0}^{t} E_{m}^{H} \mathbf{I}
$$

where I represents the identity matrix.

Step 4: Calculate Cauchy stress, ${ }^{t} \boldsymbol{\sigma}[1]$

- Calculate stress measure, $\overline{\boldsymbol{\sigma}}$

$\overline{\boldsymbol{\sigma}}=\overline{\boldsymbol{\sigma}}_{d}+\bar{\sigma}_{m} \mathbf{I}$

with

$\overline{\boldsymbol{\sigma}}_{d}=2 \mu\left({ }_{0}^{t} \mathbf{E}_{d}^{H}\right)$

$\bar{\sigma}_{m}=3 \kappa\left({ }_{0}^{t} E_{m}^{H}\right)$

where $\kappa$ represents the Bulk modulus of material, $\mu$ represents the Lame's constant as given in Eq. (14).

- Calculate Cauchy stress, ${ }^{t} \boldsymbol{\sigma}$

$$
{ }^{t} \boldsymbol{\sigma}=\frac{1}{\operatorname{det}_{0}^{t} \mathbf{X}}\left({ }_{0}^{t} \mathbf{R}\right) \overline{\boldsymbol{\sigma}}\left({ }_{0}^{t} \mathbf{R}^{T}\right)
$$


Step 5: Calculate SPK stress [1]

$$
{ }_{0}^{t} \mathbf{S}=\left(\operatorname{det}{ }_{0}^{t} \mathbf{X}\right)\left({ }_{0}^{t} \mathbf{X}^{-1}\right)\left({ }^{t} \boldsymbol{\sigma}\right)\left({ }_{0}^{t} \mathbf{X}^{-T}\right)
$$

\section{Appendix B. Peridynamic constants for 3D structures}

In order to determine peridynamic constants, two different loading cases resulting in isotropic expansion and simple shear can be considered [18] by comparing the virtual values of strain energy density in classical continuum mechanics and their peridynamic representations. The procedure for PD bond constants for 3D structures can be summarised as follows:

The virtual values of SED and volumetric strain in classical continuum mechanics for isotropic expansion is calculated in Section B.1.1. The PD representation of SED and volumetric strain for isotropic expansion is represented in Section B.1.2. By comparing the PD and the classical continuum mechanics representations, the relations for PD constants and material constants are provided in Section B.1.3.

Similarly, the virtual values of SED in classical continuum mechanics and PD for simple shear loading are presented in Sections B.2.1 and B.2.2, respectively. By comparing the PD and the classical continuum mechanics representations, the relations for PD constants and material constants are provided in Section B.2.3.

\section{B.1. Loading 1: Isotropic expansion}

B.1.1. Strain Energy Density and volumetric strain definitions in classical continuum mechanics

A loading case of isotropic expansion can be obtained by applying the following conditions

$$
\begin{aligned}
& { }_{0}^{t} u_{, x}={ }_{0}^{t} v_{, y}={ }_{0}^{t} w_{, z}=\zeta \\
& { }_{0}^{t} u_{, y}={ }_{0}^{t} u_{, z}={ }_{0}^{t} v_{, x}={ }_{0}^{t} v_{, z}={ }_{0}^{t} w_{, x}={ }_{0}^{t} w_{, y}=0
\end{aligned}
$$

Corresponding deformation gradient tensor can be calculated as

$$
{ }_{0}^{t} \mathbf{X}=\left[\begin{array}{ccc}
1+\zeta & 0 & 0 \\
0 & 1+\zeta & 0 \\
0 & 0 & 1+\zeta
\end{array}\right]
$$

By using the procedure given in Appendix A, the right stretch tensor and rotational tensor can be represented as

$$
\begin{aligned}
& { }_{0}^{t} \mathbf{U}={ }_{0}^{t} \mathbf{X} \\
& { }_{0}^{t} \mathbf{R}=\mathbf{I}
\end{aligned}
$$

Therefore, Hencky strain tensor and the SPK stress tensor can be obtained as

$$
{ }_{0}^{t} \mathbf{E}^{H}=\left[\begin{array}{ccc}
\ln (1+\zeta) & 0 & 0 \\
0 & \ln (1+\zeta) & 0 \\
0 & 0 & \ln (1+\zeta)
\end{array}\right]
$$


${ }_{0}^{t} \mathbf{S}=\frac{1}{(1+\zeta)^{2}}(3 \lambda+2 \mu)\left[\begin{array}{ccc}\ln (1+\zeta) & 0 & 0 \\ 0 & \ln (1+\zeta) & 0 \\ 0 & 0 & \ln (1+\zeta)\end{array}\right]$

For the loading case provided in Eq. (B.1), virtual strain values can be represented by using Eq. (A.18) as

$$
\begin{aligned}
& \delta\left({ }^{t+\Delta t} e_{x x}\right)=\delta\left({ }^{t+\Delta t}{ }_{t} u_{, x}\right)+\left({ }_{0}^{t} u_{, x}\right) \delta\left({ }^{t+\Delta t} u_{, x}\right)+\left({ }_{0}^{t} v_{, x}\right) \delta\left({ }^{t+\Delta t}{ }_{t} v_{, x}\right)+\left({ }_{0}^{t} w_{, x}\right) \delta\left({ }^{t+\Delta t}{ }_{t} w_{, x}\right) \\
& =(1+\zeta) \delta \zeta \\
& \delta\left({ }^{t+\Delta t} e_{y y}\right)=\delta\left({ }^{t+\Delta t} v_{, y}\right)+\left({ }_{0}^{t} v_{, y}\right) \delta\left({ }_{0} v_{, y}\right)+\left({ }_{0}^{t} u_{, y}\right) \delta\left({ }_{t}^{t+\Delta t} u_{, y}\right)+\left({ }_{0}^{t} w_{, y}\right) \delta\left({ }^{t+\Delta t} w_{, y}\right) \\
& =(1+\zeta) \delta \zeta \\
& \delta\left({ }^{t+\Delta t} e_{z z}\right)=\delta\left({ }^{t+\Delta t}{ }_{t} w_{, z}\right)+\left({ }_{0}^{t} u_{, z}\right) \delta\left({ }^{t+\Delta t} u_{, z}\right)+\left({ }_{0}^{t} v_{, z}\right) \delta\left({ }^{t+\Delta t} v_{, z}\right)+\left({ }_{0}^{t} w_{, z}\right) \delta\left({ }^{t+\Delta t}{ }_{t} w_{, z}\right) \\
& =(1+\zeta) \delta \zeta \\
& \delta\left({ }^{t+\Delta t} e_{x y}\right)=\delta\left({ }^{t+\Delta t} e_{y z}\right)=\delta\left({ }^{t+\Delta t} e_{x z}\right)=0
\end{aligned}
$$

with

$$
\begin{aligned}
& \delta\left({ }^{t+\Delta t} u_{t, x}\right)=\delta\left({ }^{t+\Delta t}{ }_{t} v_{, y}\right)=\delta\left({ }^{t+\Delta t} w_{, z}\right)=\delta \zeta \\
& \delta\left({ }^{t+\Delta t} u_{, y}\right)=\delta\left({ }^{t+\Delta t} u_{, z}\right)=\delta\left({ }^{t+\Delta t}{ }_{t} v_{, x}\right)=\delta\left({ }^{t+\Delta t}{ }_{t} v_{, z}\right)=\delta\left({ }^{t+\Delta t}{ }_{t} w_{, x}\right)=\delta\left({ }^{t+\Delta t}{ }_{t} w_{, y}\right)=0
\end{aligned}
$$

By substituting the SPK stress tensor given in Eq. (B5) and strain components given in Eq. (B6) into Eq. (A17b), the virtual value of SED in classical continuum mechanics can be calculated as

$$
\delta W^{C C M}=3(3 \lambda+2 \mu) \frac{\delta \zeta}{(1+\zeta)} \ln (1+\zeta)
$$

The volumetric strain in classical continuum mechanics for isotropic expansion can be calculated by using Hencky strain tensor given in Eq. (B4) as

$\vartheta^{C C M}={ }_{0}^{t} E_{x x}^{H}+{ }_{0}^{t} E_{y y}^{H}+{ }_{0}^{t} E_{z z}^{H}=3 \ln (1+\zeta)$

\section{B.1.2. Strain Energy Density and volumetric strain definitions in Peridynamic form}

The displacement components of material point $j$ can be expressed in terms of displacements of material point $k$ by using Taylor's series expansion by ignoring the higher-order terms as

$$
\begin{aligned}
& { }^{t} u_{(j)}={ }^{t} u_{(k)}+\left({ }_{0}^{t} u_{(k), x}\right)\left({ }^{0} x_{(j)}-{ }^{0} x_{(k)}\right)+\left({ }_{0}^{t} u_{(k), y}\right)\left({ }^{0} y_{(j)}-{ }^{0} y_{(k)}\right)+\left({ }_{0}^{t} u_{(k), z}\right)\left({ }^{0} z_{(j)}-{ }^{0} z_{(k)}\right) \\
& { }^{t} v_{(j)}={ }^{t} v_{(k)}+{ }_{0}^{t} v_{(k), x}\left({ }^{0} x_{(j)}-{ }^{0} x_{(k)}\right)+{ }_{0}^{t} v_{(k), y}\left({ }^{0} y_{(j)}-{ }^{0} y_{(k)}\right)+{ }_{0}^{t} v_{(k), z}\left({ }^{0} z_{(j)}-{ }^{0} z_{(k)}\right) \\
& { }^{t} w_{(j)}={ }^{t} w_{(k)}+{ }_{0}^{t} w_{(k), x}\left({ }^{0} x_{(j)}-{ }^{0} x_{(k)}\right)+{ }_{0}^{t} w_{(k), y}\left({ }^{0} y_{(j)}-{ }^{0} y_{(k)}\right)+{ }_{0}^{t} w_{(k), z}\left({ }^{0} z_{(j)}-{ }^{0} z_{(k)}\right)
\end{aligned}
$$

or

$$
\begin{aligned}
& \frac{{ }^{t} u_{(j)}-{ }^{t} u_{(k)}}{0} \xi \\
& { }^{t}{ }_{0}^{t} u_{(k), x} c_{x}+{ }_{0}^{t} u_{(k), y} c_{y}+{ }_{0}^{t} u_{(k), z} c_{z} \\
& \frac{{ }^{t} v_{(j)}-{ }^{t} v_{(k)}}{{ }^{0} \xi}={ }_{0}^{t} v_{(k), x} c_{x}+{ }_{0}^{t} v_{(k), y} c_{y}+{ }_{0}^{t} v_{(k), z} c_{z}
\end{aligned}
$$


$\frac{{ }^{t} w_{(j)}-{ }^{t} w_{(k)}}{{ }^{0} \xi}={ }_{0}^{t} w_{(k), x} c_{x}+{ }_{0}^{t} w_{(k), y} c_{y}+{ }_{0}^{t} w_{(k), z} c_{z}$

with

$c_{x}=\frac{{ }^{0} x_{(j)}-{ }^{0} x_{(k)}}{{ }^{0} \xi}=\sin \phi \cos \theta$

$c_{y}=\frac{{ }^{0} y_{(j)}-{ }^{0} y_{(k)}}{{ }^{0} \xi}=\sin \phi \sin \theta$

$c_{z}=\frac{{ }^{0} z_{(j)}-{ }^{0} z_{(k)}}{{ }^{0} \xi}=\cos \phi$

where $(\xi, \theta, \phi)$ serve as spherical coordinates [18].

Meanwhile, the linear bond stretch, $s$ given in Eq. (6) can be rewritten as

$s=\frac{{ }^{t} \xi}{{ }^{0} \xi}-1=\frac{\sqrt{\left({ }^{t} x_{(j)}-{ }^{t} x_{(k)}\right)^{2}+\left({ }^{t} y_{(j)}-{ }^{t} y_{(k)}\right)^{2}+\left({ }^{t} z_{(j)}-{ }^{t} z_{(k)}\right)^{2}}}{{ }^{0} \xi}-1$

or

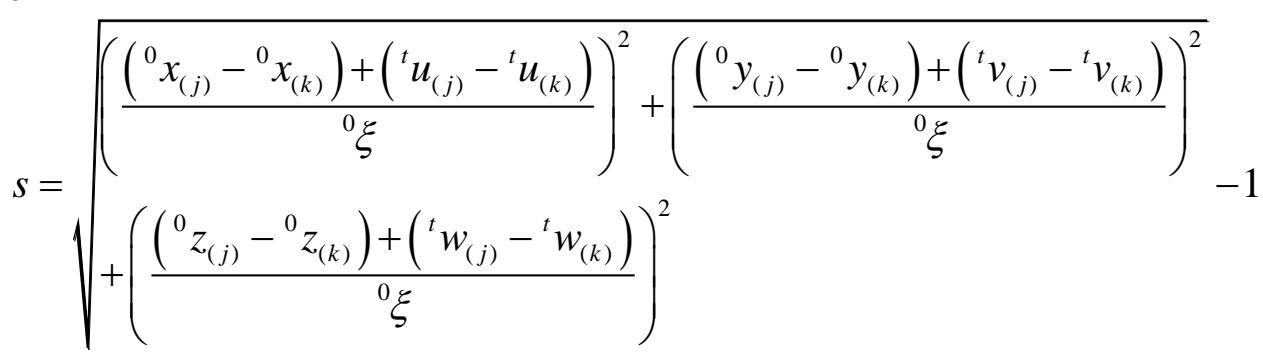

By using the relations in Eq. (B10), the linear bond stretch, $s$ given in Eq. (B11b) can be rewritten as

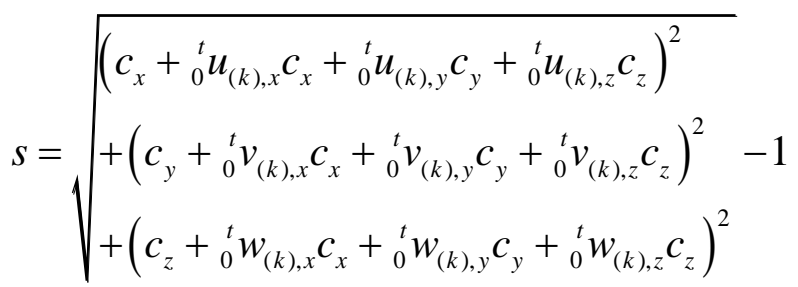

By substituting the relations given in Eq. (B1) into Eq. (B12), the linear PD bond stretch can be calculated as

$s=\zeta$

Therefore, the logarithmic bond stretch can be calculated by using Eq. (5a) as

$s_{H}=\ln (1+\zeta)$

By using the logarithmic bond stretch given in Eq. (B14), the volumetric strain in PD given in Eq. (8) can be rewritten as

$$
\vartheta_{(k)}^{P D}=d \sum_{j=1}^{N} \ln (1+\zeta) V_{(j)}
$$


By disregarding the peridynamic interactions beyond the horizon of material point $k$, the expression for $\vartheta_{(k)}^{P D}$ in Eq. (B15) can be recast as

$\vartheta_{(k)}^{P D}=d \int_{0}^{\delta_{H}} \int_{0}^{2 \pi} \int_{0}^{\pi} \ln (1+\zeta)^{0} \xi^{2} \sin \phi d \phi d \theta d^{0} \xi=d \frac{4 \pi \delta_{H}^{3}}{3} \ln (1+\zeta)$

where $\delta_{H}$ represents the horizon size on the initial configuration.

Similarly, by substituting the logarithmic stretch given in Eq. (B14) and volumetric strain in Eq. (B9) into Eq. (4a), the strain energy density in PD for isotropic expansion can be defined as

$$
W_{(k)}^{P D}=9 a \ln ^{2}(1+\zeta)+b \sum_{j=1}^{N} \ln ^{2}(1+\zeta)^{0} \xi V_{(j)}
$$

By disregarding the peridynamic interactions beyond the horizon of material point $k$, the expression for $W_{(k)}^{P D}$ can be rewritten in the integral form as

$$
W_{(k)}^{P D}=9 a \ln ^{2}(1+\zeta)+b \int_{0}^{\delta_{H}} \int_{0}^{2 \pi} \int_{0}^{\pi} \ln ^{2}(1+\zeta)^{0} \xi^{3} \sin \phi d \phi d \theta d^{0} \xi
$$

By performing the integrations in Eq. (B18), the SED in PD for isotropic expansion state can be defined as

$$
W_{(k)}^{P D}=9 a \ln ^{2}(1+\zeta)+b \pi \delta_{H}^{4} \ln ^{2}(1+\zeta)
$$

Therefore, the virtual value of strain energy density in PD can be calculated as

$$
\delta W_{(k)}^{P D}=18 a \frac{\delta \zeta}{1+\zeta} \ln (1+\zeta)+2 b \pi \delta_{H}^{4} \frac{\delta \zeta}{1+\zeta} \ln (1+\zeta)
$$

\section{B.1.3. PD constants}

By comparing volumetric strain definitions in Eq. (B9) and Eq. (B16), the PD constant, $d$ can be determined as

$$
d=\frac{9}{4 \pi \delta_{H}^{3}}
$$

By comparing the virtual value of strain energy density definitions in Eq. (B8) and Eq. (B20), the relationships between PD constants and engineering material constants can be obtained as $18 a+2 b \pi \delta_{H}^{4}=3(3 \lambda+2 \mu)$

\section{B.2. Loading 2: Simple shear}

B.2.1. Strain Energy Density and volumetric strain definitions in classical continuum mechanics

The simple shear can be obtained by assuming the following conditions

$$
\begin{aligned}
& { }_{0}^{t} u_{, x}={ }_{0}^{t} u_{, z}={ }_{0}^{t} v_{, x}={ }_{0}^{t} v_{, y}={ }_{0}^{t} v_{, z}={ }_{0}^{t} w_{, x}={ }_{0}^{t} w_{, y}={ }_{0}^{t} w_{, z}=0 \\
& { }_{0}^{t} u_{, y}=\zeta
\end{aligned}
$$

Therefore, the deformation gradient tensor can be defined as

$$
{ }_{0}^{t} \mathbf{X}=\left[\begin{array}{lll}
1 & \zeta & 0 \\
0 & 1 & 0 \\
0 & 0 & 1
\end{array}\right]
$$

By using the procedure given in Appendix A, Hencky strain tensor and Second Piola-Kirchhoff stress tensor can be obtained as 


$$
\begin{aligned}
& { }_{0}^{t} \mathbf{E}^{H}=\frac{E_{1}^{H}}{\sqrt{\zeta^{2}+4}}\left[\begin{array}{ccc}
-\zeta & 2 & 0 \\
2 & \zeta & 0 \\
0 & 0 & 0
\end{array}\right] \\
& { }_{0}^{t} \mathbf{S}=2 \mu \frac{E_{1}^{H}}{\sqrt{\zeta^{2}+4}}\left[\begin{array}{ccc}
-\zeta\left(\zeta^{2}+3\right) & \zeta^{2}+2 & 0 \\
\zeta^{2}+2 & -\zeta & 0 \\
0 & 0 & 0
\end{array}\right]
\end{aligned}
$$

with

$$
E_{1}^{H}=\frac{1}{2} \ln \left(\frac{\zeta^{2}+2}{2}+\frac{\zeta \sqrt{\zeta^{2}+4}}{2}\right)
$$

For the loading case provided in Eq. (B23), the strain components corresponding to virtual displacements given in Eq. (A18) can be calculated as

$$
\begin{aligned}
\delta\left({ }^{t+\Delta t} e_{x x}\right)= & \delta\left({ }^{t+\Delta t} e_{z z}\right)=\delta\left({ }^{t+\Delta t} e_{x z}\right)=\delta\left({ }^{t+\Delta t} e_{t} e_{y z}\right)=0 \\
\delta\left({ }^{t+\Delta t} e_{t y}\right)= & \delta\left({ }^{t+\Delta t}{ }_{t} v_{, y}\right)+{ }_{0}^{t} v_{, y} \delta\left({ }^{t+\Delta t}{ }_{t} v_{, y}\right)+{ }_{0}^{t} u_{, y} \delta\left({ }^{t+\Delta t}{ }_{t} u_{, y}\right)+{ }_{0}^{t} w_{, y} \delta\left({ }^{t+\Delta t}{ }_{t} w_{, y}\right)=\zeta \delta \zeta \\
\delta\left({ }^{t+\Delta t} e_{x y}\right)= & \frac{1}{2}\left(\delta\left({ }^{t+\Delta t} u_{, y}\right)+\delta\left({ }^{t+\Delta t} v_{, x}\right)\right) \\
& +\frac{1}{2}\left({ }_{0}^{t} u_{, x} \delta\left({ }^{t+\Delta t}{ }_{t} u_{, y}\right)+{ }_{0}^{t} v_{, x} \delta\left({ }^{t+\Delta t}{ }_{t} v_{, y}\right)+{ }_{0}^{t} u_{, y} \delta\left({ }^{t+\Delta t}{ }_{t} u_{, x}\right)\right) \\
& +\frac{1}{2}\left({ }_{0}^{t} v_{, y} \delta\left({ }^{t+\Delta t}{ }_{t} v_{, x}\right)+{ }_{0}^{t} w_{, x} \delta\left({ }^{t+\Delta t}{ }_{t} w_{, y}\right)+{ }_{0}^{t} w_{, y} \delta\left({ }^{t+\Delta t}{ }_{t} w_{, x}\right)\right) \\
= & \frac{1}{2} \delta \zeta
\end{aligned}
$$

with

$$
\begin{aligned}
& \delta\left({ }^{t+\Delta t}{ }_{t} u_{, x}\right)=\delta\left({ }^{t+\Delta t}{ }_{t} u_{, z}\right)=\delta\left({ }^{t+\Delta t}{ }_{t} v_{, x}\right)=\delta\left({ }^{t+\Delta t}{ }_{t} v_{, y}\right)=0 \\
& \delta\left({ }^{t+\Delta t}{ }_{t} v_{, z}\right)=\delta\left({ }^{t+\Delta t}{ }_{t} w_{, x}\right)=\delta\left({ }^{t+\Delta t}{ }_{t} w_{, y}\right)=\delta\left({ }^{t+\Delta t}{ }_{t} w_{, z}\right)=0 \\
& \delta\left({ }^{t+\Delta t}{ }_{t} u_{, y}\right)=\delta \zeta
\end{aligned}
$$

By using the Second Piola-Kirchhoff stress tensor given in Eq. (B25b) and the strain components given in Eq. (B26), the virtual value of strain energy density in classical continuum mechanics given in Eq. (A17b) can be calculated as

$$
\delta W^{C C M}=2 \mu \frac{1}{\sqrt{\zeta^{2}+4}} \ln \left(1+\frac{\zeta^{2}+\zeta \sqrt{\zeta^{2}+4}}{2}\right) \delta \zeta
$$

By assuming small shear strain condition, $\zeta \ll 1$, the virtual value of strain energy density in classical continuum mechanics given in Eq. (B28) can be simplified as

$$
\begin{aligned}
\delta W^{C C M} & \approx \mu\left(\zeta-\frac{1}{6} \zeta^{3}-\frac{1}{6} \zeta^{5}-\frac{1}{3} \zeta^{6}-\frac{1}{4} \zeta^{7}-\frac{1}{8} \zeta^{8}\right) \delta \zeta \\
& \approx \mu \zeta \delta \zeta+O^{3}(\zeta)
\end{aligned}
$$


where $O^{3}(\zeta)$ represents third and higher-order terms which can be neglected.

The volumetric strain in classical continuum mechanics for simple shear can be calculated by using Hencky strain tensor given in Eq. (B25a) as

$$
\vartheta^{C C M}={ }_{0}^{t} E_{x x}^{H}+{ }_{0}^{t} E_{y y}^{H}+{ }_{0}^{t} E_{z z}^{H}=0
$$

\section{B.2.2. Strain Energy Density strain definition in peridynamic form}

By using the relations given in Eq. (B23), the linear bond stretch given in Eq. (B12) can be calculated as

$$
s=\sqrt{1+2 \zeta \sin ^{2} \phi \sin \theta \cos \theta+\zeta^{2} \sin ^{2} \phi \sin ^{2} \theta}-1
$$

By assuming small shear strain, $\zeta \ll 1$, Eq. (B31) can be simplified as

$$
s \approx \zeta \sin ^{2} \phi \sin \theta \cos \theta
$$

Therefore, the logarithmic bond stretch can be calculated as

$$
s_{H} \approx \ln \left(1+\zeta \sin ^{2} \phi \sin \theta \cos \theta\right) \approx \zeta \sin ^{2} \phi \sin \theta \cos \theta-\frac{1}{2}\left(\zeta \sin ^{2} \phi \sin \theta \cos \theta\right)^{2}+\cdots
$$

By using the stretch definition given in Eq. (B33) and volumetric strain in Eq. (B30), the strain energy density in PD given in Eq. (4a) can be calculated as

$$
W_{(k)}^{P D} \approx b \sum_{j=1}^{N}\left[\zeta \sin ^{2} \phi \sin \theta \cos \theta-\frac{1}{2}\left(\zeta \sin ^{2} \phi \sin \theta \cos \theta\right)^{2}\right]^{2} \xi V_{(j)}
$$

By disregarding the peridynamic interactions beyond the horizon of material point $k$, the strain energy density given in Eq. (B34) can be recast as

$W_{(k)}^{P D} \approx b \int_{0}^{\delta_{H}} \int_{0}^{2 \pi} \int_{0}^{\pi}\left[\zeta \sin ^{2} \phi \sin \theta \cos \theta-\frac{1}{2}\left(\zeta \sin ^{2} \phi \sin \theta \cos \theta\right)^{2}\right]^{2} \xi^{0} \sin \phi d \phi d \theta d^{0} \xi$

or

$$
W_{(k)}^{P D} \approx b \int_{0}^{\delta_{H}} \int_{0}^{2 \pi} \int_{0}^{\pi} \zeta^{2} \sin ^{5} \phi \sin ^{2} \theta \cos ^{2} \theta^{0} \xi^{3} d \phi d \theta d^{0} \xi+O\left(\zeta^{3}\right)
$$

By performing integrations given in Eq. (B35b), the strain energy density in PD for the simple shear state can be calculated as

$W_{(k)}^{P D} \approx b \pi \delta_{H}^{4} \frac{1}{15} \zeta^{2}+O^{3}(\zeta)$

Therefore, the virtual value of the strain energy density in PD can be calculated as

$$
\delta W_{(k)}^{P D} \approx b \pi \delta_{H}^{4} \frac{2}{15} \zeta \delta \zeta+O^{3}(\zeta)
$$

\section{B.2.3. PD constants}

By comparing Eq. (B29) and Eq. (B37) and by neglecting third and higher-order terms, the following relations are obtained

$b \frac{2}{15} \pi \delta_{H}^{4} \zeta \delta \zeta=\mu \zeta \delta \zeta$

or

$b=\frac{15}{2} \frac{\mu}{\pi \delta_{H}^{4}}$ 
Substituting PD constant, $b$ given in Eq. (B38b) into Eq. (B22), the PD constant, $a$ can be determined as

$a=\frac{\lambda-\mu}{2}$

\section{Appendix C. Peridynamic constants for 2D structures}

Similar to 3D formulations, the PD constants for the 2D case can also be obtained by comparing the virtual values of strain energy density in classical continuum mechanics and in peridynamics in two basic loading conditions: isotropic expansion and simple shear. The procedure for PD bond constants for 2D structures can be summarised as follows:

The virtual values of SED and volumetric strain in classical continuum mechanics for isotropic expansion is calculated in Section C.1.1. The PD representation of SED and volumetric strain for isotropic expansion is represented in Section C.1.2. By comparing the PD and the classical continuum mechanics representations, the relations for PD constants and material constants are provided in Section C.1.3.

Similarly, the virtual values of SED in classical continuum mechanics and PD for simple shear loading are presented in Sections C.2.1 and C.2.2, respectively. By comparing the PD and the classical continuum mechanics representations, the relations for PD constants and material constants are provided in Section C.2.3.

\section{C.1. Loading 1: Isotropic expansion}

\section{C.1.1. Strain Energy Density and volumetric strain definitions in classical continuum} mechanics

The isotropic expansion can be obtained by assuming the following conditions

$$
\begin{aligned}
& { }_{0}^{t} u_{, x}={ }_{0}^{t} v_{, y}=\zeta \\
& { }_{0}^{t} u_{, y}={ }_{0}^{t} v_{, x}=0
\end{aligned}
$$

Therefore, the deformation gradient tensor in 2D form can be defined as

$$
{ }_{0}^{t} \mathbf{X}=\left[\begin{array}{cc}
1+\zeta & 0 \\
0 & 1+\zeta
\end{array}\right]
$$

By using the procedure given in Appendix A, Hencky strain tensor can be obtained as

$$
{ }_{0}^{t} \mathbf{E}^{H}=\left[\begin{array}{ccc}
{ }_{0}^{t} E_{x x}^{H} & 0 & 0 \\
0 & { }_{0}^{t} E_{y y}^{H} & 0 \\
0 & 0 & { }_{0}^{t} E_{z z}^{H}
\end{array}\right]=\left[\begin{array}{ccc}
\ln (1+\zeta) & 0 & 0 \\
0 & \ln (1+\zeta) & 0 \\
0 & 0 & 2(\alpha-1) \ln (1+\zeta)
\end{array}\right]
$$

where $\alpha$ is defined in Eq. (16).

Similarly, by using the procedure given in Appendix A, the Second Piola-Kirchhoff stress tensor can be obtained as 


$$
{ }_{0}^{t} \mathbf{S}=\left[\begin{array}{ccc}
\frac{2(\lambda \alpha+\mu) \ln (1+\zeta)}{(1+\zeta)^{2}} & 0 & 0 \\
0 & \frac{2(\lambda \alpha+\mu) \ln (1+\zeta)}{(1+\zeta)^{2}} & 0 \\
0 & 0 & \frac{2(\lambda \alpha+2 \mu(\alpha-1)) \ln (1+\zeta)}{(1+\zeta)^{4(\alpha-1)}}
\end{array}\right]
$$

For the loading conditions provided in Eq. (C1), the strain components corresponding to virtual displacements given in Eq. (A18) can be calculated as

$$
\begin{aligned}
& \delta\left({ }^{t+\Delta t}{ }_{t} e_{x x}\right)=\delta\left({ }^{t+\Delta t}{ }_{t} u_{, x}\right)+{ }_{0}^{t} u_{, x} \delta\left({ }^{t+\Delta t}{ }_{t} u_{, x}\right)+{ }_{0}^{t} v_{, x} \delta\left({ }^{t+\Delta t}{ }_{t} v_{, x}\right) \\
& =(1+\zeta) \delta \zeta \\
& \delta\left({ }^{t+\Delta t} e_{y y}\right)=\delta\left({ }^{t+\Delta t}{ }_{t} v_{, y}\right)+{ }_{0}^{t} v_{, y} \delta\left({ }^{t+\Delta t}{ }_{t} v_{, y}\right)+{ }_{0}^{t} u_{, y} \delta\left({ }^{t+\Delta t} u_{, y}\right) \\
& =(1+\zeta) \delta \zeta \\
& \delta\left({ }^{t+\Delta t} e_{x y}\right)=\frac{1}{2}\left(\delta\left({ }^{t+\Delta t} u_{, y}\right)+\delta\left({ }^{t+\Delta t} v_{, x}\right)\right) \\
& +\frac{1}{2}\left({ }_{0}^{t} u_{, x} \delta\left({ }^{t+\Delta t} u_{, y}\right)+{ }_{0}^{t} v_{, x} \delta\left({ }^{t+\Delta t}{ }_{t} v_{, y}\right)+{ }_{0}^{t} u_{, y} \delta\left({ }^{t+\Delta t} u_{, x}\right)+{ }_{0}^{t} v_{, y} \delta\left({ }^{t+\Delta t}{ }_{t} v_{, x}\right)\right) \\
& =0
\end{aligned}
$$

with

$$
\delta\left({ }^{t+\Delta t} u_{, x}\right)=\delta\left({ }^{t+\Delta t}{ }_{t} v_{, y}\right)=\delta \zeta, \delta\left({ }^{t+\Delta t} u_{, y}\right)=\delta\left({ }^{t+\Delta t} v_{, x}\right)=0
$$

Since ${ }_{0}^{t} S_{z z} \delta\left({ }^{t+\Delta t} e_{z z}\right)=0$ for both plane strain and plane stress conditions, the virtual value of SED in classical continuum mechanics can be calculated as

$$
\delta W^{C C M}={ }_{0}^{t} S_{x x} \delta\left({ }^{t+\Delta t} e_{x x}\right)+{ }_{0}^{t} S_{y y} \delta\left({ }^{t+\Delta t} e_{y y}\right)+2{ }_{0}^{t} S_{x y} \delta\left({ }^{t+\Delta t} e_{x y}\right)
$$

By substituting the Second Piola-Kirchhoff stress tensor given in Eq. (C4) and strain components given in Eq. (C5) into Eq. (C6), the virtual value of SED can be calculated as

$$
\delta W^{C C M}=4(\lambda \alpha+\mu) \frac{\delta \zeta}{(1+\zeta)} \ln (1+\zeta)
$$

The 2D volumetric strain in classical continuum mechanics can be calculated from Eq. (C3) as $\vartheta^{C C M}={ }_{0}^{t} E_{x x}^{H}+{ }_{0}^{t} E_{y y}^{H}=2 \ln (1+\zeta)$

\section{C.1.2. Strain Energy Density and volumetric strain definitions in Peridynamic form}

The displacement components of material point $j$ can be expressed in terms of displacements of material point $k$ by using Taylor's series expansion by ignoring the higher-order terms as

$$
\begin{aligned}
& { }^{t} u_{(j)}={ }^{t} u_{(k)}+{ }_{0}^{t} u_{(k), x}\left({ }^{0} x_{(j)}-{ }^{0} x_{(k)}\right)+{ }_{0}^{t} u_{(k), y}\left({ }^{0} y_{(j)}-{ }^{0} y_{(k)}\right) \\
& { }^{t} v_{(j)}={ }^{t} v_{(k)}+{ }_{0}^{t} v_{(k), x}\left({ }^{0} x_{(j)}-{ }^{0} x_{(k)}\right)+{ }_{0}^{t} v_{(k), y}\left({ }^{0} y_{(j)}-{ }^{0} y_{(k)}\right)
\end{aligned}
$$

or 
$\frac{{ }^{t} u_{(j)}-{ }^{t} u_{(k)}}{{ }^{0} \xi}={ }_{0}^{t} u_{(k), x} \cos \varphi+{ }_{0}^{t} u_{(k), y} \sin \varphi$

$\frac{{ }^{t} v_{(j)}-{ }^{t} v_{(k)}}{{ }^{0} \xi}={ }_{0}^{t} v_{(k), x} \cos \varphi+{ }_{0}^{t} v_{(k), y} \sin \varphi$

with

$\cos \varphi=\frac{{ }^{0} x_{(j)}-{ }^{0} x_{(k)}}{{ }^{0} \xi}$

$\sin \varphi=\frac{{ }^{0} v_{(j)}-{ }^{0} v_{(k)}}{{ }^{0} \xi}$

Meanwhile, the linear bond stretch, $s$ given in Eq. (6) can be rewritten as

$s=\frac{\sqrt{\left({ }^{t} x_{(j)}-{ }^{t} x_{(k)}\right)^{2}+\left({ }^{t} y_{(j)}-{ }^{t} y_{(k)}\right)^{2}}}{{ }^{0} \xi}-1=\sqrt{\left(\frac{\left({ }^{t} x_{(j)}-{ }^{t} x_{(k)}\right.}{{ }^{0} \xi}\right)^{2}+\left(\frac{{ }^{t} y_{(j)}-{ }^{t} y_{(k)}}{{ }^{0} \xi}\right)^{2}}-1$

or

$s=\sqrt{\left(\frac{\left({ }^{0} x_{(j)}-{ }^{0} x_{(k)}\right)+\left({ }^{t} u_{(j)}-{ }^{t} u_{(k)}\right)}{{ }^{0} \xi}\right)^{2}+\left(\frac{\left({ }^{0} y_{(j)}-{ }^{0} y_{(k)}\right)+\left({ }^{t} v_{(j)}-{ }^{t} v_{(k)}\right)}{{ }^{0} \xi}\right)^{2}}-1$

By using the relations in Eqs. (C9c) and (C9d), the linear bond stretch, $s$ given in Eq. (C10b) can be rewritten as

$s=\sqrt{\left(\cos \varphi+{ }_{0}^{t} u_{(k), x} \cos \varphi+{ }_{0}^{t} u_{(k), y} \sin \varphi\right)^{2}+\left(\sin \varphi+{ }_{0}^{t} v_{(k), x} \cos \varphi+{ }_{0}^{t} v_{(k), y} \sin \varphi\right)^{2}}-1$

By substituting conditions given in Eq. (C1) into Eq. (C11), the PD linear stretch can be calculated as

$s=\zeta$

Therefore, the logarithmic bond stretch can be calculated as

$s_{H}=\ln (1+\zeta)$

By using the logarithmic bond stretch given in Eq. (C13), the PD volumetric strain given in Eq. (8) can be rewritten as

$\vartheta_{(k)}^{P D}=d \sum_{j=1}^{N} \ln (1+\zeta) V_{(j)}$

By disregarding the peridynamic interactions beyond the horizon of material point $k$, the expression for $\vartheta_{(k)}^{P D}$ in Eq. (C14) can be recast as

$\vartheta_{(k)}^{P D}=d h \int_{0}^{\delta_{H}} \int_{0}^{2 \pi} \ln (1+\zeta)^{0} \xi d^{0} \xi d \varphi=d \pi h \delta_{H}^{2} \ln (1+\zeta)$

where $h$ represents the plate thickness on the initial configuration.

Similarly, by substituting the logarithmic stretch given in Eq. (C13) and volumetric strain in Eq. C8) into Eq. (4a), the strain energy density in PD in the isotropic expansion state can also be defined as 
$W_{(k)}^{P D}=4 a \ln ^{2}(1+\zeta)+b \sum_{j=1}^{N} \ln ^{2}(1+\zeta)^{0} \xi V_{(j)}$

By disregarding the peridynamic interactions beyond the horizon of material point $k$, the expression for $W_{(k)}$ can be recast in the integral form as

$W_{(k)}^{P D}=4 a \ln ^{2}(1+\zeta)+b h \int_{0}^{\delta_{H}} \int_{0}^{2 \pi} \ln ^{2}(1+\zeta)^{0} \xi^{2} d \varphi d^{0} \xi$

or

$W_{(k)}^{P D}=4 a \ln ^{2}(1+\zeta)+b \frac{2 \pi h \delta_{H}^{3}}{3} \ln ^{2}(1+\zeta)$

Therefore, the virtual value of strain energy density in PD can be calculated as

$\delta W_{(k)}^{P D}=8 a \frac{\delta \zeta}{1+\zeta} \ln (1+\zeta)+b \frac{4 \pi h \delta_{H}^{3}}{3} \frac{\delta \zeta}{1+\zeta} \ln (1+\zeta)$

\section{C.1.3. PD constants}

By comparing Eq. (C8) and Eq. (C15), the PD constant, $d$ can be determined as

$d=\frac{2}{\pi h \delta_{H}^{2}}$

By comparing Eq. (C7) and Eq. (C18), the relationships between PD constants and engineering material constants can be obtained as

$2 a+b \frac{\pi h \delta_{H}^{3}}{3}=\lambda \alpha+\mu$

C.2. Loading 2: Simple shear

\section{C.2.1. Strain Energy Density definition in classical continuum mechanics}

The simple shear can be obtained by assuming the following conditions

${ }_{0}^{t} u_{, x}={ }_{0}^{t} v_{, x}={ }_{0}^{t} v_{, y}=0$

${ }_{0}^{t} u_{, y}=\zeta$

Therefore, the deformation gradient tensor can be defined as

${ }_{0}^{t} \mathbf{X}=\left[\begin{array}{ll}1 & \zeta \\ 0 & 1\end{array}\right]$

By using the procedure given in Appendix A, Hencky strain tensor and Second Piola-Kirchhoff stress tensor can be obtained as

${ }_{0}^{t} \mathbf{E}^{H}=\frac{E_{1}^{H}}{\sqrt{\zeta^{2}+4}}\left[\begin{array}{ccc}-\zeta & 2 & 0 \\ 2 & \zeta & 0 \\ 0 & 0 & 0\end{array}\right]$ 
${ }_{0}^{t} \mathbf{S}=2 \mu \frac{E_{1}^{H}}{\sqrt{\zeta^{2}+4}}\left[\begin{array}{ccc}-\zeta\left(\zeta^{2}+3\right) & \zeta^{2}+2 & 0 \\ \zeta^{2}+2 & -\zeta & 0 \\ 0 & 0 & 0\end{array}\right]$

where

$E_{1}^{H}=\frac{1}{2} \ln \left(\frac{\zeta^{2}+2}{2}+\frac{\zeta \sqrt{\zeta^{2}+4}}{2}\right)$

Note that in simple shear loading condition, the component ${ }_{0}^{t} E_{z z}^{H}=0$ and ${ }_{0}^{t} S_{z z} \delta\left({ }^{t+\Delta t} e_{z z}\right)=0$ in both plane stress and plane strain conditions.

For the loading case provided in Eq. (C.21), the strain components corresponding to virtual displacements can be defined as

$$
\begin{aligned}
\delta\left({ }^{t+\Delta t} e_{x x}\right)= & \delta\left({ }^{t+\Delta t} u_{, x}\right)+{ }_{0}^{t} u_{, x} \delta\left({ }^{t+\Delta t}{ }_{t} u_{, x}\right)+{ }_{0}^{t} v_{, x} \delta\left({ }^{t+\Delta t} v_{, x}\right)=0 \\
\delta\left({ }^{t+\Delta t} e_{y y}\right)= & \delta\left({ }^{t+\Delta t}{ }_{t} v_{, y}\right)+{ }_{0}^{t} v_{, y} \delta\left({ }^{t+\Delta t}{ }_{t} v_{, y}\right)+{ }_{0}^{t} u_{, y} \delta\left({ }^{t+\Delta t} u_{, y}\right)=\zeta \delta \zeta \\
\delta\left({ }^{t+\Delta t} e_{x y}\right)= & \frac{1}{2}\left(\delta\left({ }^{t+\Delta t}{ }_{t} u_{, y}\right)+\delta\left({ }^{t+\Delta t}{ }_{t} v_{, x}\right)\right) \\
& +\frac{1}{2}\left({ }_{0}^{t} u_{, x} \delta\left({ }^{t+\Delta t} u_{, y}\right)+{ }_{0}^{t} v_{, x} \delta\left({ }^{t+\Delta t}{ }_{t} v_{, y}\right)+{ }_{0}^{t} u_{, y} \delta\left({ }_{t}^{t+\Delta t} u_{, x}\right)+{ }_{0}^{t} v_{, y} \delta\left({ }^{t+\Delta t} v_{, x}\right)\right) \\
= & \frac{1}{2} \delta \zeta
\end{aligned}
$$

with

$$
\begin{aligned}
& \delta\left({ }^{t+\Delta t}{ }_{t} u_{, x}\right)=\delta\left({ }^{t+\Delta t}{ }_{t} v_{, x}\right)=\delta\left({ }^{t+\Delta t}{ }_{t} v_{, y}\right)=0 \\
& \delta\left({ }^{t+\Delta t}{ }_{t} u_{, y}\right)=\zeta
\end{aligned}
$$

By substituting the Second Piola-Kirchhoff stress tensor given in Eq. (C23b) and the strain components given in Eq. (C24) into Eq. (C6), the virtual value of strain energy density in classical continuum mechanics can be calculated as

$$
\begin{aligned}
\delta W^{C C M} & ={ }_{0}^{t} S_{x x} \delta\left({ }_{t}^{t+\Delta t} e_{x x}\right)+{ }_{0}^{t} S_{y y} \delta\left({ }_{t}^{t+\Delta t} e_{y y}\right)+2{ }_{0}^{t} S_{x y} \delta\left({ }^{t+\Delta t} e_{x y}\right) \\
& =2 \mu \frac{1}{\sqrt{\zeta^{2}+4}} \ln \left(1+\frac{\zeta^{2}+\zeta \sqrt{\zeta^{2}+4}}{2}\right) \delta \zeta
\end{aligned}
$$

Similar to 3D structures, by assuming that $\zeta \ll 1$, the virtual value of strain energy density in classical continuum mechanics given in Eq. (C25) can be simplified as

$$
\delta W^{C C M} \approx \mu\left(\zeta-\frac{1}{6} \zeta^{3}-\frac{1}{6} \zeta^{5}-\frac{1}{3} \zeta^{6}-\frac{1}{4} \zeta^{7}-\frac{1}{8} \zeta^{8}\right) \delta \zeta \approx \mu \zeta \delta \zeta+O^{3}(\zeta)
$$

The volumetric strain in classical continuum mechanics for simple shear can be calculated by using Hencky strain tensor given in Eq. (C23a) as 
$\vartheta^{C C M}={ }_{0}^{t} E_{x x}^{H}+{ }_{0}^{t} E_{y y}^{H}+{ }_{0}^{t} E_{z z}^{H}=0$

\section{C.2.2. Strain Energy Density strain definition in Peridynamic form}

In peridynamics, by using conditions given in Eq. (C21), the linear stretch given in Eq. (C11) can be calculated for $\zeta \ll 1$ as

$s=\sqrt{1+2 \zeta \sin \varphi \cos \varphi+\zeta^{2} \sin ^{2} \varphi}-1 \approx \zeta \sin \varphi \cos \varphi$

Therefore, the logarithmic stretch given in Eq. (5a) can be calculated as

$s_{H} \approx \ln (1+\zeta \sin \varphi \cos \varphi) \approx \zeta \sin \varphi \cos \varphi-\frac{1}{2}(\zeta \sin \varphi \cos \varphi)^{2}$

By using the stretch given in Eq. (C29) and volumetric strain in Eq. (C27), the strain energy density in PD given in Eq. (4a) can be calculated as

$W_{(k)}^{P D} \approx b \sum_{j=1}^{N} \zeta^{2} \sin ^{2} \varphi \cos ^{2} \varphi^{0} \xi V_{(j)}+O^{3}(\zeta)$

The strain energy density given in Eq. (C30) can be recast as

$W_{(k)}^{P D}=b h \int_{0}^{\delta_{H}} \int_{0}^{2 \pi} \zeta^{2} \sin ^{2} \varphi \cos ^{2} \varphi^{0} \xi^{2} d^{0} \xi d \varphi+O^{3}(\zeta)$

By performing integrations given in Eq. (C31), the strain energy density in PD for the simple shear state can be calculated as

$W_{(k)}^{P D} \approx \frac{1}{12} b \pi h \delta_{H}^{3} \zeta^{2}+O^{3}(\zeta)$

Therefore, the virtual value of the strain energy density in PD can be calculated as

$\delta W_{(k)}^{P D} \approx \frac{1}{6} b \pi h \delta_{H}^{3} \zeta \delta \zeta+O^{3}(\zeta)$

\section{C.2.3. PD constants}

By comparing Eq. (C26) and Eq. (C33), the following relations are obtained

$\frac{1}{6} b \pi h \delta_{H}^{3} \zeta \delta \zeta=\mu \zeta \delta \zeta$

or

$b=\frac{6 \mu}{\pi h \delta_{H}^{3}}$

Substituting PD constant, $b$ given in Eq. (C34b) into Eq. (C20), the PD constant, $a$ can be determined as

$a=\frac{\lambda \alpha-\mu}{2}$

\section{Appendix D. PD constants for $1 D$ structures}

The procedure for PD bond constants for 1D structures can be summarised as follows:

The virtual values of SED in classical continuum mechanics for uniform stretch is calculated in Section D.1. The PD representation of SED and volumetric strain for uniform stretch is represented in Section D.2. By comparing the PD and the classical continuum mechanics representations, the relations for PD constants and material constants are provided in Section. D.3. 


\section{D.1. Strain Energy Density definitions in classical continuum mechanics}

In order to determine the PD constant, a bar can be assumed to be subjected to a uniform stretch of

${ }_{0}^{t} u_{, x}=\zeta$

which results in the deformation gradient as

${ }_{0}^{t} \mathbf{X}=1+\zeta$

By using the procedure as given in Appendix A, Hencky strain tensor and the SPK stress tensor can be

$$
\begin{aligned}
& { }_{0}^{t} \mathbf{E}^{H}=\ln (1+\zeta) \\
& { }_{0}^{t} \mathbf{S}=E \frac{1}{(1+\zeta)^{2}} \ln (1+\zeta)
\end{aligned}
$$

For the loading case provided in Eq. (D1), virtual strain values can be represented by using Eq. (A.18) as

$$
\delta\left({ }^{t+\Delta t} e_{x x}\right)=\delta\left({ }^{t+\Delta t}{ }_{t} u_{, x}\right)+{ }_{0}^{t} u_{, x} \delta\left({ }^{t+\Delta t}{ }_{t} u_{, x}\right)=\delta \zeta+\zeta \delta \zeta=(1+\zeta) \delta \zeta
$$

with

$\delta\left({ }^{t+\Delta t} u_{, x}\right)=\delta \zeta$

Therefore, the virtual value of strain energy density in classical continuum mechanics can be calculated as

$$
\delta W^{C C M}={ }_{0}^{t} S_{x x} \delta\left({ }^{t+\Delta t} e_{x x}\right)=E \frac{\delta \zeta}{1+\zeta} \ln (1+\zeta)
$$

\section{D.2. Strain Energy Density in Peridynamic form}

The relation between the relative coordinate of two material points in the initial and deformed configurations can be assumed as

$$
\left({ }^{0} x_{(j)}-{ }^{0} x_{(k)}\right)\left({ }^{t} x_{(j)}-{ }^{k} x_{(k)}\right)>0 \quad \forall k \neq j
$$

or

$$
\frac{{ }^{0} x_{(j)}-{ }^{0} x_{(k)}}{{ }^{0} \xi}=\frac{{ }^{t} x_{(j)}-{ }^{k} x_{(k)}}{{ }^{t} \xi} \quad \forall k \neq j
$$

Moreover, the linear bond stretch given in Eq. (6) can be rewritten for 1D structure as

$$
s=\frac{\left|{ }^{t} x_{(j)}-{ }^{t} x_{(k)}\right|}{{ }^{0} \xi}-1=\frac{{ }^{t} x_{(j)}-{ }^{t} x_{(k)}}{{ }^{0} \xi} \frac{{ }^{t} x_{(j)}-{ }^{t} x_{(k)}}{{ }^{t} \xi}-1
$$

Therefore, by using the relation given in Eq. (D6b), the linear bond stretch given in Eq. (D7) can be rewritten as

$$
s=\frac{{ }^{0} x_{(j)}-{ }^{0} x_{(k)}+u_{(j)}-u_{(k)}}{{ }^{0} \xi} \frac{x_{(j)}-{ }^{0} x_{(k)}}{{ }^{0} \xi}-1=\frac{u_{(j)}-u_{(k)}}{{ }^{0} \xi} \frac{{ }^{0} x_{(j)}-{ }^{0} x_{(k)}}{{ }^{0} \xi}
$$

Meanwhile, the displacement components of material point $j$ can be expressed in terms of displacements of material point $k$ by using Taylor's series expansion by ignoring the higherorder terms as 
${ }^{t} u_{(j)}={ }^{t} u_{(k)}+{ }_{0}^{t} u_{(k), x}\left({ }^{0} x_{(j)}-{ }^{0} x_{(k)}\right)$

or

$\frac{{ }^{t} u_{(j)}-{ }^{t} u_{(k)}}{{ }^{0} \xi}={ }_{0}^{t} u_{(k), x} \frac{\left({ }^{0} x_{(j)}-{ }^{0} x_{(k)}\right)}{{ }^{0} \xi}$

Therefore, by utilizing the relation given in Eq. (D9b), the linear bond stretch given in Eq. (D8) can be rewritten as

$s={ }_{0}^{t} u_{(k), x}\left(\frac{{ }^{0} x_{(j)}-{ }^{0} x_{(k)}}{{ }^{0} \xi}\right)^{2}={ }_{0}^{t} u_{(k), x}$

By substituting the relation given in Eq. (D1) into Eq. (D10), the linear PD bond stretch can be obtained as

$s=\zeta$

Therefore, the logarithmic bond stretch can be obtained as

$s_{H}=\ln (1+\zeta)$

By utilizing the logarithmic bond stretch given in Eq. (D12), the strain energy density given in Eq. (4b) can be written as

$W_{(k)}^{P D}=b \sum_{j=1}^{N} \ln ^{2}(1+\zeta)^{0} \xi V_{(j)}$

The SED given in Eq. (D13) can be rewritten in an integral form with respected to the initial configuration as

$W_{(k)}^{P D}=2 b A \int_{0}^{\delta_{H}} \ln ^{2}(1+\zeta)^{0} \xi d^{0} \xi$

where $A$ represents the cross-section area on the initial configuration.

By performing integration given in Eq. (D14), the SED for the bar can be calculated as

$W_{(k)}^{P D}=b A \delta_{H}^{2} \ln ^{2}(1+\zeta)$

Therefore, the virtual value of strain energy density in PD can be calculated as

$\delta W_{(k)}^{P D}=2 b A \delta_{H}^{2} \frac{\delta \zeta}{1+\zeta} \ln (1+\zeta)$

D.3. PD constant

By comparing Eq. (D5) and Eq. (D116), the PD bond constant can be defined as

$b=\frac{E}{2 A \delta_{H}^{2}}$

\section{Appendix E. Updating loading conditions}

E.1. One dimensional problem

In the example of the 1D structure presented in Section 6.1, the constant volume is assumed in both FEA and PD analyses. Therefore, the body force, which is applied to the material point located at the right end of the bar, remains constant during the simulations.

E.2. Two-dimensional problems

As provided in Eq. (A2), the external work caused by surface forces is updated during nonlinear simulations. In Section 6.2, the plate is subjected to constant pressures. The pressure is defined 
as normal to the applied surfaces. Due to large deformations, the normal direction of these surfaces can be changed. Therefore, the direction of loading can also be changed. In PD analysis, the pressure loading conditions are converted to body forces. Therefore, the body forces applied on a material point as shown in Fig. E1 can be updated as

$$
\begin{aligned}
& { }^{0} \mathbf{b}_{(k)}=-\frac{p\left({ }^{0} S_{(k)}\right)}{{ }^{0} V_{(k)}}{ }^{0} \mathbf{n}_{(k)} \\
& { }^{t} \mathbf{b}_{(k)}=-\frac{p\left({ }^{t} S_{(k)}\right)}{{ }^{t} V_{(k)}}{ }^{t} \mathbf{n}_{(k)}
\end{aligned}
$$

where ${ }^{0} \mathbf{b}_{(k)},{ }^{0} V_{(k)}$ and ${ }^{t} \mathbf{b}_{(k)},{ }^{t} V_{(k)}$ represent the body force and volume of material point $k$ at $t=0$ and time $t$, respectively. Moreover, ${ }^{t} S_{(k)},{ }^{0} \mathbf{n}_{(k)}$ and ${ }^{t} S_{(k)},{ }^{t} \mathbf{n}_{(k)}$ represent the surface area and normal vector of material point $k$ on which the pressure is applied at $t=0$ and time $t$, respectively as shown in Fig. E1.
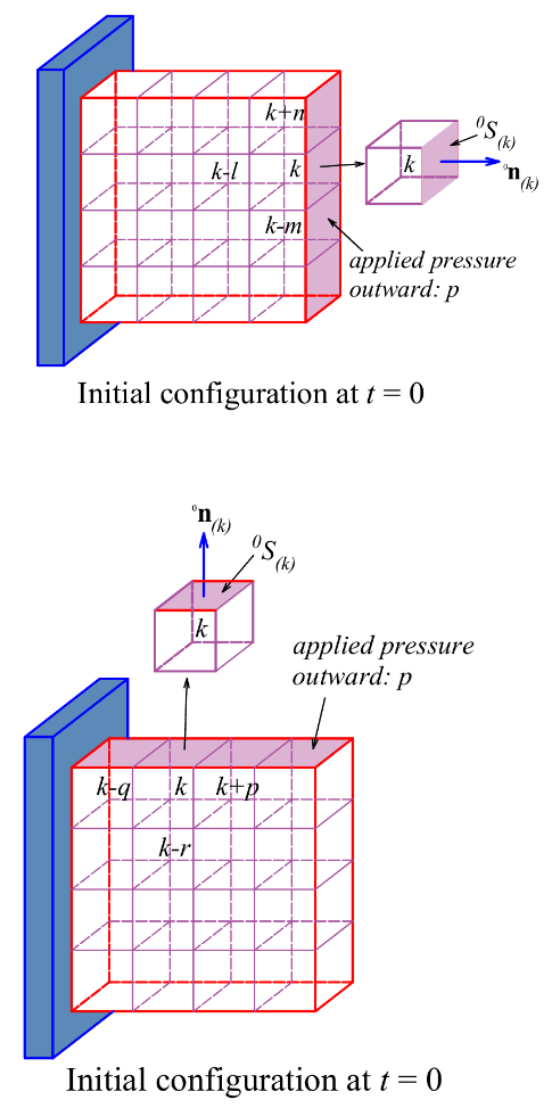
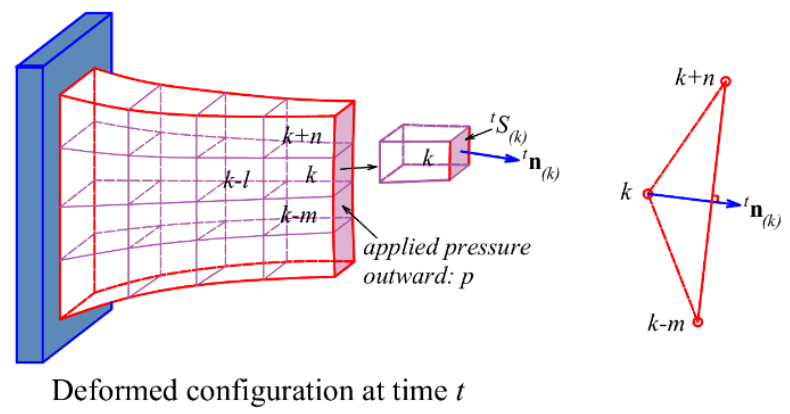

(a)

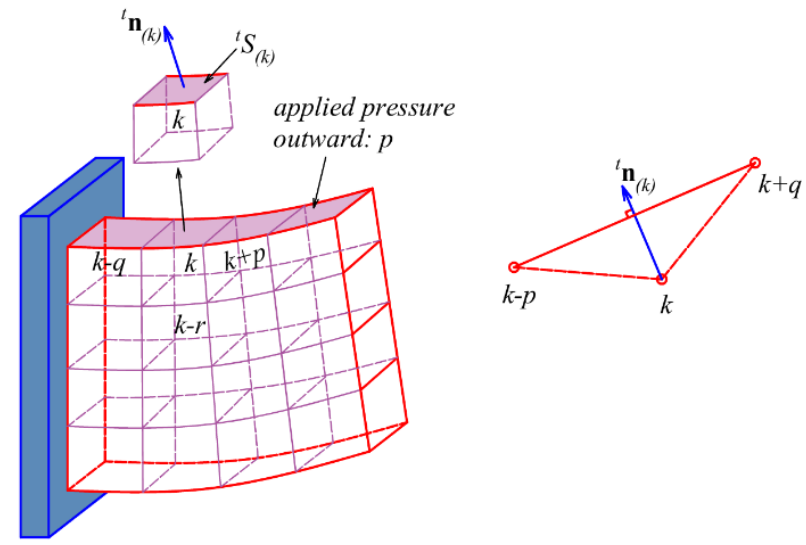

Deformed configuration at time $t$

(b)

Fig. E1. Undeformed and deformed configuration of plate (a) plate under tension loading (b) plate under shear loading

In the following sections, the calculations of the volume of material point, ${ }^{t} V_{(k)}$, surface area, ${ }^{t} S_{(k)}$ and normal vector ${ }^{t} \mathbf{n}_{(k)}$ at time $t$ are presented.

\section{Calculation of volume ${ }^{t} V_{(k)}$}

The volume of a material point at the current time $t$ can be calculated as 
${ }^{t} V_{(k)}={ }^{t} h_{(k)}{ }^{t} A_{(k)}$

where ${ }^{t} h_{(k)}$ and ${ }^{t} A_{(k)}$ represent plate thickness and area of material point $k$ at the current time $t$, respectively. The area ${ }^{t} A_{(k)}$ can be calculated as [1]

${ }^{t} A_{(k)}={ }^{0} A_{(k)} \operatorname{det}{ }_{0}^{t} \mathbf{X}_{(k)}$

where ${ }^{0} A_{(k)}$ represents the area of material point in the initial configuration which can be calculated as

${ }^{0} A_{(k)}=\left(\Delta^{0} x\right)^{2}$

where $\Delta^{0} x$ represents the the initial mesh size of the PD model.

In Eq. (E3), det ${ }_{0}^{t} \mathbf{X}_{(k)}$ represents determinant of the deformation gradient matrix. The deformation gradient ${ }_{0}^{t} \mathbf{X}_{(k)}$ can be calculated as described in Appendix F.

The plate thickness, ${ }^{t} h_{(k)}$ in Eq. (E2) can be calculated from the definition of Hencky strain in the thickness direction as [52]

${ }_{0}^{t} E_{z z(k)}^{H}=\ln \left(\frac{{ }^{t} h_{(k)}}{{ }^{0} h_{(k)}}\right)$

which can also be written as

${ }^{t} h_{(k)}={ }^{0} h_{(k)}\left(e^{{ }^{t} E_{z z(k)}^{H}}\right)$

where ${ }^{0} h_{(k)}$ represents the initial plate thickness, ${ }_{0}^{t} E_{z z(k)}^{H}$ represents Hencky strain in the thickness direction. This strain component can be calculated as follows;

First, the deformation gradient ${ }_{0}^{t} \mathbf{X}_{(k)}$ is calculated as described in Appendix F. Next, by using the procedure given in Appendix A, the Hencky strain tensor is calculated. Finally, Hencky strain in the thickness, direction ${ }_{0}^{t} E_{z z(k)}^{H}$ can be calculated as

${ }_{0}^{t} E_{z z(k)}^{H}=(\alpha-1)\left({ }_{0}^{t} E_{x x(k)}^{H}+{ }_{0}^{t} E_{y y(k)}^{H}\right)$

with $\alpha$ represents a parameter for plane stress or plane strain as given in Eq. (17).

Note that the calculation of current thickness is only used to update the loading conditions. The interaction forces are calculated using the initial configuration.

Calculation of surface area ${ }^{t} S_{(k)}$

The surface area, ${ }^{t} S_{(k)}$, of material point $k$ can be calculated as

For Fig. E1(a):

${ }^{t} S_{(k)}={ }^{t} \Delta y_{(k)}{ }^{t} h_{(k)}$

with

$$
\begin{aligned}
& { }^{t} \Delta y_{(k)}=\frac{1}{2}\left({ }^{t} \xi_{(k)(k+n)}+{ }^{t} \xi_{(k)(k-m)}\right) \\
& { }^{t} \xi_{(k)(k+n)}=\sqrt{\left({ }^{t} x_{(k)}-{ }^{t} x_{(k+n)}\right)^{2}+\left({ }^{t} y_{(k)}-{ }^{t} y_{(k+n)}\right)^{2}} \\
& { }^{t} \xi_{(k)(k-m)}=\sqrt{\left({ }^{t} x_{(k)}-{ }^{t} x_{(k-m)}\right)^{2}+\left({ }^{t} y_{(k)}-{ }^{t} y_{(k-m)}\right)^{2}}
\end{aligned}
$$


For Fig. E1(b):

${ }^{t} S_{(k)}={ }^{t} \Delta x_{(k)}{ }^{t} h_{(k)}$

with

$$
\begin{aligned}
& { }^{t} \Delta x_{(k)}=\frac{1}{2}\left({ }^{t} \xi_{(k)(k+p)}+{ }^{t} \xi_{(k)(k-q)}\right) \\
& { }^{t} \xi_{(k)(k+p)}=\sqrt{\left({ }^{t} x_{(k)}-{ }^{t} x_{(k+p)}\right){ }^{2}+\left({ }^{t} y_{(k)}-{ }^{t} y_{(k+p)}\right)^{2}} \\
& { }^{t} \xi_{(k)(k-q)}=\sqrt{\left({ }^{t} x_{(k)}-{ }^{t} x_{(k-q)}\right)^{2}+\left({ }^{t} y_{(k)}-{ }^{t} y_{(k-q)}\right)^{2}}
\end{aligned}
$$

The plate thickness, ${ }^{t} h_{(k)}$ in Eq. (E7a) and (E8a) can be calculated by using Eq. (E5).

\section{Update normal vector ${ }^{t} \mathbf{n}_{(k)}$}

The direction of the normal vector of the surface ${ }^{t} S_{(k)}$ can be changed due to large deformations. Therefore, the normal vector ${ }^{t} \mathbf{n}_{(k)}$ of surface ${ }^{t} S_{(k)}$ can be updated by satisfying following relations

For a material point shown in Fig. E1(a):

$$
\begin{aligned}
& \left({ }^{t} \mathbf{x}_{(k+n)}-{ }^{t} \mathbf{x}_{(k-m)}\right) \cdot{ }^{t} \mathbf{n}_{(k)}=0 \\
& \left({ }^{t} \mathbf{x}_{(k-l)}-{ }^{t} \mathbf{x}_{(k)}\right) \cdot{ }^{t} \mathbf{n}_{(k)}<0 \\
& \left|{ }^{t} \mathbf{n}_{(k)}\right|=1
\end{aligned}
$$

For a material point shown in Fig. E1(b):

$$
\begin{aligned}
& \left({ }^{t} \mathbf{x}_{(k+p)}-{ }^{t} \mathbf{x}_{(k-q)}\right) \cdot{ }^{t} \mathbf{n}_{(k)}=0 \\
& \left({ }^{t} \mathbf{x}_{(k-r)}-{ }^{t} \mathbf{x}_{(k)}\right) \cdot{ }^{t} \mathbf{n}_{(k)}<0 \\
& \left|{ }^{t} \mathbf{n}_{(k)}\right|=1
\end{aligned}
$$

The procedure for updating loading conditions for 2D problems can be described as follows; Step 1. Updating volume ${ }^{t} V_{(k)}$ at each time step

1.1. Calculate shape function, $\mathbf{K}_{(k)}$ by using Eq. (F3)

1.2. Calculate the displacement gradient, $\nabla_{0}^{t} \mathbf{u}_{(k)}$ by using Eq. (F2)

1.3. Calculate the deformation gradient, ${ }_{0}^{t} \mathbf{X}_{(k)}$ by using Eq. (F1)

1.4. Calculate Hencky strain tensor ${ }_{0}^{t} \mathbf{E}_{(k)}^{H}$ by using the procedure given in Appendix A

1.5. Calculate the strain component in the thickness direction, ${ }_{0}^{t} E_{z z(k)}^{H}$ by using Eq. (E6)

1.6. Update thickness, ${ }^{t} h_{(k)}$ by using Eq. (E5b)

1.7. Update area, ${ }^{t} A_{(k)}$ by using Eq. (E3)

1.8. Update volume, ${ }^{t} V_{(k)}$ by using Eq. (E2)

Step 2. Updating surface area ${ }^{t} S_{(k)}$ at each time step

2.1. Update deformed configuration 
2.2. Calculate, ${ }^{t} \Delta x_{(k)}$ or ${ }^{t} \Delta y_{(k)}$ by using Eq. (E7b) or (E8b)

2.3. Calculate ${ }^{t} S_{(k)}$ by using Eq. (E7a) or (E8a)

Step 3. Updating normal vector ${ }^{t} \mathbf{n}_{(k)}$ at each time step by using relations in Eq. (E9) or (E10)

Step 4. Update body force ${ }^{t} \mathbf{b}_{(k)}$ by using Eq. (E1)

The procedure from Step 1 to Step 4 is repeated for all material points that loading is applied.

E.3. Three-dimensional problems

In Section 6.3, the 3D structure is subjected to distributed force along the right edge as shown in Fig. 26. The load is applied in the form of body force by considering the change of volume of materials. Therefore, as shown in Fig. E2, the material points located along the edge of the PD model will be subjected to body forces as

$$
\begin{aligned}
& { }^{0} \mathbf{b}_{(k)}=\frac{{ }^{0} \Delta y_{(k)}}{{ }^{0} V_{(k)}} \mathbf{f}=\left(\frac{{ }^{0} \Delta y_{(k)}}{{ }^{0} V_{(k)}} f_{x}, \frac{{ }^{0} \Delta y_{(k)}}{{ }^{0} V_{(k)}} f_{y}, \frac{{ }^{0} \Delta y_{(k)}}{{ }^{0} V_{(k)}} f_{z}\right)=\left({ }^{0} b_{x},{ }^{0} b_{y},{ }^{0} b_{z}\right) \\
& { }^{t} \mathbf{b}_{(k)}=\frac{{ }^{t} \Delta y_{(k)}}{{ }^{t} V_{(k)}} \mathbf{f}=\left(\frac{{ }^{t} \Delta y_{(k)}}{{ }^{t} V_{(k)}} f_{x}, \frac{{ }^{t} \Delta y_{(k)}}{{ }^{t} V_{(k)}} f_{y}, \frac{{ }^{t} \Delta y_{(k)}}{{ }^{t} V_{(k)}} f_{z}\right)=\left({ }^{t} b_{x},{ }^{t} b_{y},{ }^{t} b_{z}\right)
\end{aligned}
$$

with

${ }^{t} V_{(k)}={ }^{0} V_{(k)} \operatorname{det}{ }_{0}^{t} \mathbf{X}_{(k)}$

where ${ }^{0} \Delta y_{(k)}$ and ${ }^{t} \Delta y_{(k)}$ represent mesh sizes along the edge of the PD model at initial and current times as shown in Fig. E2. The mesh size ${ }^{t} \Delta y_{(k)}$ in deformed configuration at time $t$ can be calculated by using Eq. (E7b).

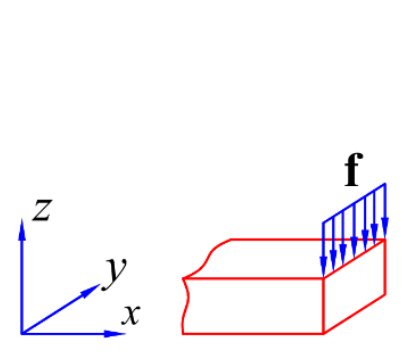

(a)

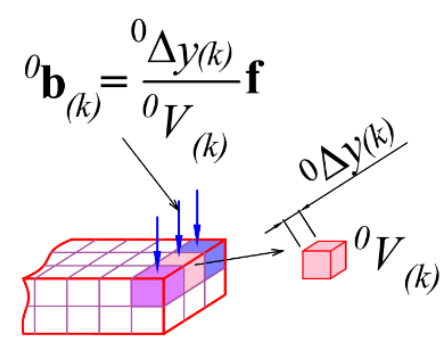

(b)

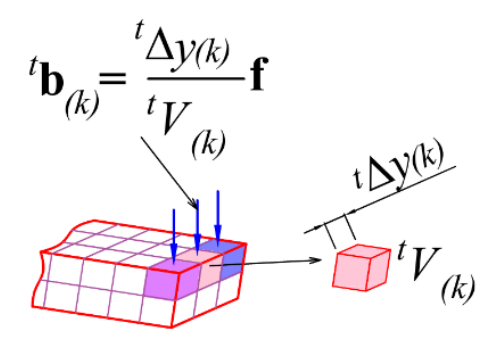

(c)

Fig. E2. Apply line distributed load in PD model (a) geometry, (b) body forces at $t=0$, (c) body forces at time $t$

The procedure for updating loading conditions for 3D problems can be described as follows;

Step 1. Updating volume ${ }^{t} V_{(k)}$ at each time step

1.1. Calculate shape function, $\mathbf{K}_{(k)}$ by using Eq. (F3)

1.2. Calculate the displacement gradient, $\nabla_{0}^{t} \mathbf{u}_{(k)}$ by using Eq. (F2)

1.3. Calculate the deformation gradient, ${ }_{0}^{t} \mathbf{X}_{(k)}$ by using Eq. (F1)

1.4. Update volume, ${ }^{t} V_{(k)}$ by using Eq. (E11c)

Step 2. Update ${ }^{t} \Delta y_{(k)}$ by using Eq. (E7b)

Step 3. Update body force ${ }^{t} \mathbf{b}_{(k)}$ by using Eq. (E11) 
The procedure from Step 1 to Step 3 is repeated for all material points that are subjected to distributed load.

\section{Appendix F: Calculation of the deformation gradient in PD}

The deformation gradient is used to update the change of area for 2D structure and the change of volume for 3D structures as provided in Eq. (E3) and (E11c). The deformation gradient in PD can be defined as

$$
{ }_{0}^{t} \mathbf{X}_{(k)}=\nabla_{0}^{t} \mathbf{u}_{(k)}+\mathbf{I}
$$

where $\nabla_{0}^{t} \mathbf{u}_{(k)}$ represents the displacement gradient, $\mathbf{I}$ is the identity matrix.

The displacement gradient, $\nabla_{0}^{t} \mathbf{u}_{(k)}$, can be calculated according to Breitenfeld et al. [68] as

$$
\nabla_{0}^{t} \mathbf{u}_{(k)}=\left[\sum_{j=1}^{N}\left(\frac{\delta_{H}}{\left|{ }^{0} \mathbf{x}_{(j)}-{ }^{0} \mathbf{x}_{(k)}\right|}\right)^{2}\left({ }^{t} \mathbf{u}_{(j)}-{ }^{t} \mathbf{u}_{(k)}\right) \otimes\left({ }^{0} \mathbf{x}_{(j)}-{ }^{0} \mathbf{x}_{(k)}\right) V_{(j)}\right] \mathbf{K}_{(k)}
$$

where $\mathbf{K}_{(k)}$ represents shape function which can be defined as [68]

$$
\mathbf{K}_{(k)}=\left[\sum_{j=1}^{N}\left(\frac{\delta_{H}}{\left|{ }^{0} \mathbf{x}_{(j)}-{ }^{0} \mathbf{x}_{(k)}\right|}\right)^{2}\left({ }^{0} \mathbf{x}_{(j)}-{ }^{0} \mathbf{x}_{(k)}\right) \otimes\left({ }^{0} \mathbf{x}_{(j)}-{ }^{0} \mathbf{x}_{(k)}\right) V_{(j)}\right]^{-1}
$$

\section{Appendix G: Balance laws}

In this section, the balances of linear momentum and angular momentum are presented. The balance of linear momentum can be represented as [18]

$\dot{\mathbf{L}}=\mathbf{F}$

where $\mathbf{L}$ represents linear momentum, $\mathbf{F}$ represents the total force acting on the PD model. The linear momentum for a fixed set of particles in volume $V$ can be represented as [18]

$\mathbf{L}=\int_{V} \rho(\mathbf{x}) \dot{\mathbf{u}}(\mathbf{x}, t) d V$

The total force, $\mathbf{F}$, can be represented as

$$
\mathbf{F}=\int_{V} \mathbf{b}(\mathbf{x}, t) d V+\int_{V} \int_{H}\left(\mathbf{t}\left(\mathbf{u}^{\prime}-\mathbf{u}, \mathbf{x}^{\prime}-\mathbf{x}, t\right)-\mathbf{t}^{\prime}\left(\mathbf{u}-\mathbf{u}^{\prime}, \mathbf{x}-\mathbf{x}^{\prime}, t\right)\right) d H d V
$$

The balance of angular momentum can be represented as [18]

$$
\dot{\mathbf{H}}_{0}=\boldsymbol{\Pi}_{0}
$$

where $\mathbf{H}_{0}$ represents angular momentum, $\boldsymbol{\Pi}_{0}$ represents the total torque acting on the PD model. The angular momentum, $\mathbf{H}_{0}$ for a fixed set of particles in volume $V$ can be represented as [18]

$$
\mathbf{H}_{0}=\int_{V} \mathbf{y}(\mathbf{x}, t) \times \rho(\mathbf{x}) \dot{\mathbf{u}}(\mathbf{x}, t) d V
$$

The torque, $\boldsymbol{\Pi}_{0}$, can be represented as

$$
\boldsymbol{\Pi}_{0}=\int_{V} \mathbf{y}(\mathbf{x}, t) \times \mathbf{b}(\mathbf{x}, t) d V+\int_{V} \int_{H} \mathbf{y}(\mathbf{x}, t) \times\left(\mathbf{t}\left(\mathbf{u}^{\prime}-\mathbf{u}, \mathbf{x}^{\prime}-\mathbf{x}, t\right)-\mathbf{t}^{\prime}\left(\mathbf{u}-\mathbf{u}^{\prime}, \mathbf{x}-\mathbf{x}^{\prime}, t\right)\right) d H d V
$$

Because $\mathbf{t}\left(\mathbf{u}^{\prime}-\mathbf{u}, \mathbf{x}^{\prime}-\mathbf{x}, t\right)=\mathbf{t}^{\prime}\left(\mathbf{u}-\mathbf{u}^{\prime}, \mathbf{x}-\mathbf{x}^{\prime}, t\right)=\mathbf{0}$ for $\mathbf{x}^{\prime} \notin H$, Eq. (G3) and (G6) can be rewritten as [18]

$$
\mathbf{F}=\int_{V} \mathbf{b}(\mathbf{x}, t) d V+\int_{V}\left(\mathbf{t}\left(\mathbf{u}^{\prime}-\mathbf{u}, \mathbf{x}^{\prime}-\mathbf{x}, t\right)-\mathbf{t}^{\prime}\left(\mathbf{u}-\mathbf{u}^{\prime}, \mathbf{x}-\mathbf{x}^{\prime}, t\right)\right) d V^{\prime} d V
$$




$$
\boldsymbol{\Pi}_{0}=\int_{V} \mathbf{y}(\mathbf{x}, t) \times \mathbf{b}(\mathbf{x}, t) d V+\int_{V} \int_{V} \mathbf{y}(\mathbf{x}, t) \times\left(\mathbf{t}\left(\mathbf{u}^{\prime}-\mathbf{u}, \mathbf{x}^{\prime}-\mathbf{x}, t\right)-\mathbf{t}^{\prime}\left(\mathbf{u}-\mathbf{u}^{\prime}, \mathbf{x}-\mathbf{x}^{\prime}, t\right)\right) d V^{\prime} d V
$$

If the parameters $\mathbf{x}$ and $\mathbf{x}^{\prime}$ are exchanged in the second integrals in the right-hand side of Eqs. (G7) and (G8), the following relations are obtained [18]

$$
\begin{aligned}
& \int_{V} \int_{V} \mathbf{t}\left(\mathbf{u}^{\prime}-\mathbf{u}, \mathbf{x}^{\prime}-\mathbf{x}, t\right) d V^{\prime} d V=\int_{V} \int_{V} \mathbf{t}^{\prime}\left(\mathbf{u}-\mathbf{u}^{\prime}, \mathbf{x}-\mathbf{x}^{\prime}, t\right) d V d V^{\prime} \\
& \iint_{V} \mathbf{y}^{\prime}\left(\mathbf{x}^{\prime}, t\right) \times \mathbf{t}\left(\mathbf{u}^{\prime}-\mathbf{u}, \mathbf{x}^{\prime}-\mathbf{x}, t\right) d V^{\prime} d V=\iint_{V} \int_{V} \mathbf{y}(\mathbf{x}, t) \times \mathbf{t}^{\prime}\left(\mathbf{u}-\mathbf{u}^{\prime}, \mathbf{x}-\mathbf{x}^{\prime}, t\right) d V d V^{\prime}
\end{aligned}
$$

Therefore, by using the relation given in Eqs. (G9-10), the total force given in Eq. (G7) and the torque given in Eq. (G8) can be simplified as

$$
\begin{aligned}
& \mathbf{F}=\int_{V} \mathbf{b}(\mathbf{x}, t) d V \\
& \boldsymbol{\Pi}_{0}=\int_{V} \mathbf{y}(\mathbf{x}, t) \times \mathbf{b}(\mathbf{x}, t) d V+\int_{V} \int_{V}\left(\mathbf{y}(\mathbf{x}, t)-\mathbf{y}^{\prime}\left(\mathbf{x}^{\prime}, t\right)\right) \times \mathbf{t}\left(\mathbf{u}^{\prime}-\mathbf{u}, \mathbf{x}^{\prime}-\mathbf{x}, t\right) d V^{\prime} d V
\end{aligned}
$$

By substituting Eq. (G2) and (G11) into Eq. (G1), the balance of linear moment can be represented as

$\int_{V}(\rho(\mathbf{x}) \ddot{\mathbf{u}}(\mathbf{x}, t)-\mathbf{b}(\mathbf{x}, t)) d V=0$

According to Madenci and Oterkus [18], Eq. (G13) is automatically satisfied for arbitrary force density vectors $\mathbf{t}\left(\mathbf{u}^{\prime}-\mathbf{u}, \mathbf{x}^{\prime}-\mathbf{x}, t\right)$ and $\mathbf{t}^{\prime}\left(\mathbf{u}-\mathbf{u}^{\prime}, \mathbf{x}-\mathbf{x}^{\prime}, t\right)$. Therefore, the balance of linear momentum is satisfied with this PD model.

By substituting Eq. (G5) and (G12) into Eq. (G4), the balance of angular moment can be represented as

$$
\int_{V} \mathbf{y}(\mathbf{x}, t) \times(\rho(\mathbf{x}) \ddot{\mathbf{u}}(\mathbf{x}, t)-\mathbf{b}(\mathbf{x}, t)) d V=\int_{V} \int_{V}\left(\mathbf{y}(\mathbf{x}, t)-\mathbf{y}^{\prime}\left(\mathbf{x}^{\prime}, t\right)\right) \times \mathbf{t}\left(\mathbf{u}^{\prime}-\mathbf{u}, \mathbf{x}^{\prime}-\mathbf{x}, t\right) d V^{\prime} d V
$$

In the ordinary state-based PD model, the vector of PD force density $\mathbf{t}\left(\mathbf{u}^{\prime}-\mathbf{u}, \mathbf{x}^{\prime}-\mathbf{x}, t\right)$ parallels to the deformed configuration. Therefore, the following relation is obtained

$$
\int_{V} \int_{V}\left(\mathbf{y}(\mathbf{x}, t)-\mathbf{y}^{\prime}\left(\mathbf{x}^{\prime}, t\right)\right) \times \mathbf{t}\left(\mathbf{u}^{\prime}-\mathbf{u}, \mathbf{x}^{\prime}-\mathbf{x}, t\right) d V^{\prime} d V=0
$$

Therefore, by using the relation given in Eq. (G15), the balance of angular momentum given in Eq. (G14) can be simplified as

$$
\int_{V} \mathbf{y}(\mathbf{x}, t) \times(\rho(\mathbf{x}) \ddot{\mathbf{u}}(\mathbf{x}, t)-\mathbf{b}(\mathbf{x}, t)) d V=0
$$

By utilizing Eq. (G13), the relation given in Eq. (G16) is satisfied. Therefore, the balance of angular momentum is satisfied. 


\section{References}

[1] Bathe, K.-J., Finite element procedures. 2006: Klaus-Jurgen Bathe.

[2] Anand, L., On H. Hencky's approximate strain-energy function for moderate deformations. Journal of Applied Mechanics, 1979. 46(1): p. 78-82.

[3] Bonet, J. and R.D. Wood, Nonlinear continuum mechanics for finite element analysis. 1997: Cambridge university press.

[4] Bathe, K.-j. and S. Bolourchi, A geometric and material nonlinear plate and shell element. Computers structures, 1980. 11(1-2): p. 23-48.

[5] Bathe, K.-J. and E.N. Dvorkin, On the automatic solution of nonlinear finite element equations. Computers Structures, 1983. 17(5-6): p. 871-879.

[6] Hughes, T.J., K.S. Pister, and R.L. Taylor, Implicit-explicit finite elements in nonlinear transient analysis. Computer Methods in Applied Mechanics Engineering, 1979. 17: p. 159-182.

[7] Wilson, E., I. Farhoomand, and K. Bathe, Nonlinear dynamic analysis of complex structures. Earthquake Engineering Structural Dynamics, 1972. 1(3): p. 241-252.

[8] Bathe, K.J. and A.P. Cimento, Some practical procedures for the solution of nonlinear finite element equations. Computer methods in applied mechanics engineering, 1980. 22(1): p. 59-85.

[9] Bhatti, M.A., Advanced topics in finite element analysis of structures: with Mathematica and MATLAB computations. 2006: John Wiley \& Sons, Inc.

[10] Silling, S.A., Reformulation of elasticity theory for discontinuities and long-range forces. Journal of the Mechanics and Physics of Solids, 2000. 48(1): p. 175-209.

[11] Silling, S.A. and E. Askari, A meshfree method based on the peridynamic model of solid mechanics. Computers \& structures, 2005. 83(17-18): p. 1526-1535.

[12] Silling, S.A., et al., Peridynamic states and constitutive modeling. Journal of Elasticity, 2007. 88(2): p. 151-184.

[13] Silling, S.A. and R. Lehoucq, Peridynamic theory of solid mechanics, in Advances in applied mechanics. 2010, Elsevier. p. 73-168.

[14] Wang, Y., et al., A 3-D conjugated bond-pair-based peridynamic formulation for initiation and propagation of cracks in brittle solids. International Journal of Solids Structures, 2018. 134: p. 89-115.

[15] Wang, Y., X. Zhou, and Y. Shou, The modeling of crack propagation and coalescence in rocks under uniaxial compression using the novel conjugated bond-based peridynamics. International Journal of Mechanical Sciences, 2017. 128: p. 614-643.

[16] Bergel, G.L. and S. Li, The total and updated lagrangian formulations of state-based peridynamics. Computational Mechanics, 2016. 58(2): p. 351-370.

[17] Nikravesh, S. and W. Gerstle, Improved state-based peridynamic lattice model including elasticity, plasticity and damage. CMES-Comput Model Eng, 2018. 116(3): p. 323-347.

[18] Madenci, E. and E. Oterkus, Peridynamic Theory and Its Applications. Peridynamic Theory and Its Applications. 2014: Springer.

[19] Javili, A., et al., Peridynamics review. Mathematics Mechanics of Solids, 2018: p. 1081286518803411.

[20] Foster, J.T., S.A. Silling, and W.W. Chen, Viscoplasticity using peridynamics. International journal for numerical methods in engineering, 2010. 81(10): p. 12421258.

[21] Mitchell, J.A., A non-local, ordinary-state-based viscoelasticity model for peridynamics. Sandia National Lab Report, 2011. 8064: p. 1-28. 
[22] Madenci, E. and S. Oterkus, Ordinary state-based peridynamics for plastic deformation according to von Mises yield criteria with isotropic hardening. Journal of the Mechanics Physics of Solids, 2016. 86: p. 192-219.

[23] Madenci, E. and S. Oterkus, Ordinary state-based peridynamics for thermoviscoelastic deformation. Engineering Fracture Mechanics, 2017. 175: p. 31-45.

[24] Oterkus, S., Peridynamics for the solution of multiphysics problems. 2015, The University of Arizona: The University of Arizona.

[25] Han, S., et al. Equivalent acceleration assessment of JEDEC moisture sensitivity levels using peridynamics. in 2015 IEEE 65th Electronic Components and Technology Conference (ECTC). 2015. IEEE.

[26] Madenci, E. and S. Oterkus, Peridynamics for coupled field equations. Handbook of Peridynamic Modeling, 2016: p. 489-531.

[27] Oterkus, S., E. Madenci, and E. Oterkus, Fully coupled poroelastic peridynamic formulation for fluid-filled fractures. Engineering geology, 2017. 225: p. 19-28.

[28] Wang, Y., X. Zhou, and M. Kou, An improved coupled thermo-mechanic bond-based peridynamic model for cracking behaviors in brittle solids subjected to thermal shocks. European Journal of Mechanics-A/Solids, 2019. 73: p. 282-305.

[29] Wang, H., E. Oterkus, and S. Oterkus, Predicting fracture evolution during lithiation process using peridynamics. Engineering Fracture Mechanics, 2018. 192: p. 176-191.

[30] Wang, H., E. Oterkus, and S. Oterkus, Peridynamic modelling of fracture in marine lithium-ion batteries. Ocean Engineering, 2018. 151: p. 257-267.

[31] Oterkus, S., E. Madenci, and A. Agwai, Fully coupled peridynamic thermomechanics. Journal of the Mechanics Physics of Solids, 2014. 64: p. 1-23.

[32] Alpay, S. and E. Madenci. Crack growth prediction in fully-coupled thermal and deformation fields using peridynamic theory. in 54th AIAA/ASME/ASCE/AHS/ASC structures, structural dynamics, and materials conference. 2013.

[33] Gao, Y. and S. Oterkus, Ordinary state-based peridynamic modelling for fully coupled thermoelastic problems. Continuum Mechanics Thermodynamics, 2019. 31(4): p. $907-$ 937.

[34] Askari, E., et al. Peridynamics for multiscale materials modeling. in Journal of Physics: Conference Series. 2008. IOP Publishing.

[35] Bobaru, F. and Y.D. Ha, Adaptive refinement and multiscale modeling in 2D peridynamics. Journal for Multiscale Computational Engineering, 2011: p. 635-659.

[36] Oterkus, E., Peridynamic theory for modeling three-dimensional damage growth in metallic and composite structures. 2010, The University of Arizona: The University of Arizona.

[37] Oterkus, E. and E. Madenci. Peridynamic theory for damage initiation and growth in composite laminate. in Key Engineering Materials. 2012. Trans Tech Publ.

[38] Hu, W., Y.D. Ha, and F. Bobaru, Peridynamic model for dynamic fracture in unidirectional fiber-reinforced composites. Computer Methods in Applied Mechanics Engineering, 2012. 217: p. 247-261.

[39] De Meo, D., N. Zhu, and E. Oterkus, Peridynamic modeling of granular fracture in polycrystalline materials. Journal of Engineering Materials Technology, 2016. 138(4): p. 041008.

[40] Gao, Y. and S. Oterkus, Fully coupled thermomechanical analysis of laminated composites by using ordinary state based peridynamic theory. Composite Structures, 2019. 207: p. 397-424.

[41] Gao, Y. and S. Oterkus, Peridynamic analysis of marine composites under shock loads by considering thermomechanical coupling effects. Journal of Marine Science Engineering, 2018. 6(2): p. 38. 
[42] Diyaroglu, C., E. Oterkus, and S. Oterkus, An Euler-Bernoulli beam formulation in an ordinary state-based peridynamic framework. Mathematics Mechanics of Solids, 2019. 24(2): p. 361-376.

[43] Nguyen, C.T. and S. Oterkus, Peridynamics formulation for beam structures to predict damage in offshore structures. Ocean Engineering, 2019. 173: p. 244-267.

[44] Diyaroglu, C., et al., Peridynamics for bending of beams and plates with transverse shear deformation. International Journal of Solids Structures, 2015. 69: p. 152-168.

[45] Nguyen, C.T. and S. Oterkus, Peridynamics for the thermomechanical behavior of shell structures. Engineering Fracture Mechanics, 2019: p. 106623.

[46] Kilic, B. and E. Madenci, Coupling of peridynamic theory and the finite element method. Journal of mechanics of materials structures, 2010. 5(5): p. 707-733.

[47] Oterkus, E., et al., Combined finite element and peridynamic analyses for predicting failure in a stiffened composite curved panel with a central slot. Composite Structures, 2012. 94(3): p. 839-850.

[48] Liu, W. and J.-W. Hong, A coupling approach of discretized peridynamics with finite element method. Computer methods in applied mechanics engineering, 2012. 245: p. 163-175.

[49] Bie, Y., X. Cui, and Z. Li, A coupling approach of state-based peridynamics with nodebased smoothed finite element method. Computer Methods in Applied Mechanics Engineering, 2018. 331: p. 675-700.

[50] Macek, R.W. and S.A. Silling, Peridynamics via finite element analysis. Finite Elements in Analysis Design, 2007. 43(15): p. 1169-1178.

[51] Han, S., et al. Peridynamic direct concentration approach by using ANSYS. in 2016 IEEE 66th Electronic Components and Technology Conference (ECTC). 2016. IEEE.

[52] Diyaroglu, C., et al., Peridynamic modeling of diffusion by using finite-element analysis. IEEE Transactions on Components, Packaging Manufacturing Technology, 2017. 7(11): p. 1823-1831.

[53] Yang, Z., et al., Implementation of peridynamic beam and plate formulations in finite element framework. Continuum Mechanics Thermodynamics, 2019. 31(1): p. 301-315.

[54] Kalthoff, J.F., Shadow optical analysis of dynamic shear fracture. Optical Engineering, 1988. 27(10): p. 271035.

[55] Kalthoff, J.F., Modes of dynamic shear failure in solids. International Journal of Fracture, 2000. 101(1-2): p. 1-31.

[56] Kalthoff, J. and S. Winkler, Failure mode transition at high rates of shear loading. DGM Informationsgesellschaft mbH, Impact Loading Dynamic Behavior of Materials, 1988. 1: p. 185-195.

[57] Jenq, Y. and S.P. Shah, Mixed-mode fracture of concrete. International Journal of Fracture, 1988. 38(2): p. 123-142.

[58] Foster, J.T., S.A. Silling, and W. Chen, An energy based failure criterion for use with peridynamic states. International Journal for Multiscale Computational Engineering, 2011. 9(6).

[59] Wang, Y., X. Zhou, and X. Xu, Numerical simulation of propagation and coalescence of flaws in rock materials under compressive loads using the extended non-ordinary state-based peridynamics. Engineering Fracture Mechanics, 2016. 163: p. 248-273.

[60] Underwood, P., Dynamic relaxation. Computational method for transient analysis, 1986. 1: p. 245-263.

[61] Kilic, B. and E. Madenci, An adaptive dynamic relaxation method for quasi-static simulations using the peridynamic theory. Theoretical Applied Fracture Mechanics, 2010. 53(3): p. 194-204. 
[62] Oterkus, S. and E. Madenci, Peridynamics for antiplane shear and torsional deformations. Journal of Mechanics of Materials Structures, 2015. 10(2): p. 167-193.

[63] Borden, M.J., et al., A phase-field description of dynamic brittle fracture. Computer Methods in Applied Mechanics Engineering, 2012. 217: p. 77-95.

[64] Le, Q. and F. Bobaru, Surface corrections for peridynamic models in elasticity and fracture. Computational Mechanics, 2018. 61(4): p. 499-518.

[65] Butt, S.N., J.J. Timothy, and G. Meschke, Wave dispersion and propagation in statebased peridynamics. Computational Mechanics, 2017. 60(5): p. 725-738.

[66] Dipasquale, D., M. Zaccariotto, and U. Galvanetto, Crack propagation with adaptive grid refinement in $2 D$ peridynamics. International Journal for Numerical Methods in Engineering, 2014. 190(1-2): p. 1-22.

[67] Hesch, C., et al., A framework for polyconvex large strain phase-field methods to fracture. Computer Methods in Applied Mechanics Engineering, 2017. 317: p. 649683.

[68] Breitenfeld, M., et al., Non-ordinary state-based peridynamic analysis of stationary crack problems. Computer Methods in Applied Mechanics Engineering, 2014. 272: p. 233-250. 